NATIONAL AERONAUTICS AND SPACE ADMINISTRATION

Technical Memorandum 33-720

\title{
Data-Derived Symbol Synchronization of MASK and QASK Signa/s
}

\author{
Marvin K. Simon
}

(NASA-CR-141316) DATA-DERIVED SYMBOL

SYNCHRONIZATION OF MASK AND QASK SIGNALS

(Jet propulsion Lab.) 87 p HC $\$ 4.75$
N75- 14926 $63 / 32 \quad 06580$

JET PROPULSION LABORATORY

CALIFORNIA INSTITUTE OF TECHNOLOGY

PASADENA, CALIFORNIA

December 15, 1974 
NATIONAL AERONAUTICS AND SPACE ADMINISTRATION

Technical Memorandum 33-720

\title{
Data-Derived Symbol Synchronization of MASK and QASK Signals
}

\author{
Marvin K. Simon
}

JET PROPULSION LABORATORY CALIFORNIA INSTITUTE OF TECHNOLOGY

PASADENA, CALIFORNIA

December 15, 1974 
Prepared Under Contract No, NAS 7.100 National Aeronautics and Space Administration 


\section{PREFACE}

The work described in this report was performed by the Telecommunications Division of the Jet Propulsion Laboratory.

\section{PRECEDING PAGE BLANR NOT FILMGD}




\section{CONTENTS}

A. Introduction .......................... 1

B. Description of Loop Operation .................. I

C. System Analysis .......................... 4

D. Application of the Fokker-Planck Equation ............. 10

E. Error Probability Performance ................. I3

F. Modification of the Mask Symbol Sync Loop for a

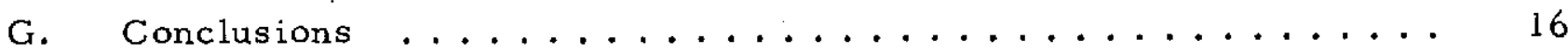

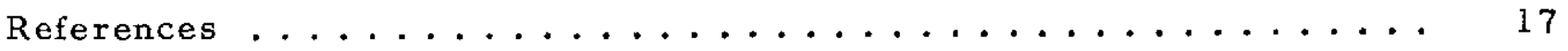

Appendices

A. Evaluation of the Loop Nonlinearity $g(\lambda) \ldots \ldots \ldots$ A $-\ldots$

B. Evaluation of the Loop Spectrum $S(0,0) \ldots \ldots$ B-I

C. Slope of the Loop S-Curve at the Origin .......... C-1

D. Derivation of the Conditional Error Probability $P_{E}(\lambda)$

for a MASK System with a Fixed Symbol Sync Error..... D-1

E. Evaluation of the Moments of a Various Functions Arising from the Passage of a Signal Plus Noise through a K-Level Quantizer ............... E-1

\section{FIGURES}

1. Data-Transition Type Symbol of Synchronizer for MASK Signals .......................... E-13

2. Equivalent Data-Transition Tracking Loop Model ...................... E-14

3a. Normalized Loop Nonlinearity vs Normalized Symbol Sync Error $; \xi=1 \ldots \ldots \ldots$ E-15

3b. Normalized Loop Nonlinearity vs Normalized Symbol Sync Error; $\xi=1 \ldots \ldots \ldots$ E-16

4a. Normalized Loop Nonlinearity vs Normalized Symbol Sync Error; $\xi=0.5 \ldots \ldots \ldots$ E-1 7 
CONTENTS (contd)

\section{FIGURES (contd)}

4b. Normalized Loop Nonlinearity vs Normalized

Symbol Sync Error; $\xi=0.5 \ldots \ldots \ldots$ E-18

5. Normalized Noise Spectral Density vs

Signal-to-Noise Ratio; $\mathrm{K}=2 \ldots \ldots$ E-19

6. Normalized Noise Spectral Density vs

Signal-to-Noise Ratio; $K=4 \ldots \ldots$ E -20

7. Normalized Noise Spectral Density vs

Signal-to-Noise Ratio; $\mathrm{K}=8 \ldots \ldots \ldots$ E-2l

8. Slope of S-Curve at Origin vs Signal-to-Noise

Ratio; $\mathrm{K}=2 \ldots \ldots \ldots \ldots$ E- . . . . . . . . . . . . 22

9. Slope of S-Curve at Origin vs Signal-to-Noise

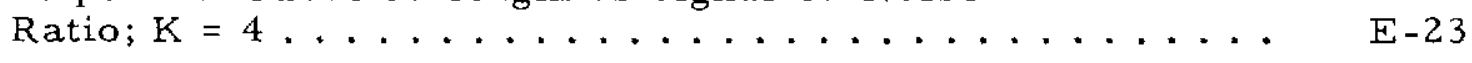

10. Slope of S-Curve at Origin vs Signal-to-Noise

Ratio $\mathrm{K}=8 \ldots \ldots \ldots \ldots$ E -24

11. RMS Symbol Sync Jitter vs Signal-to-Noise

Ratio $\mathrm{K}=2 \ldots \ldots \ldots \ldots \ldots \ldots$ E -25

12. RMS Symbol Sync Jitter vs Signal-to-Noise

Ratio $\mathrm{K}=4 \ldots \ldots \ldots \ldots \ldots$ E-26

13. RMS Symbol Sync Jitter vs Signal-to-Noise

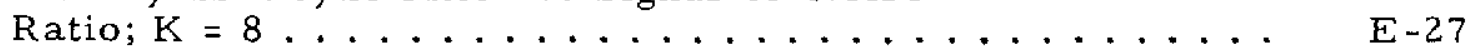

14. Error Probability Pexformance of MASK System with

Noisy Symbol Sync Reference; $\mathrm{K}=2 \ldots \ldots$ E-28

15. Error Probability Performance of MASK System with

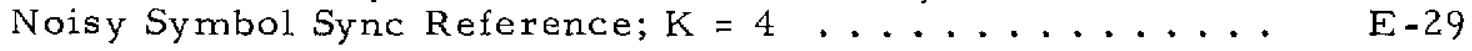

16. Data-Transition Type of Symbol Synchronizer

for QASK Signals .................. E E 30

17. Error Probability Performance of QASK System with

Noisy Symbol Sync Reference $K^{2}=4$

(Quadriphase Signaling) ................ E-31

18. Error Probability Performance of QASK System with

Noisy Symbol Sync Reference; $\mathrm{K}^{2}=16 \ldots \ldots$ E -32 


\begin{abstract}
Much has been said in the literature with regard to the problem of establishing symbol synchronization in binary baseband digital communication systems. By comparison, the literature is virtually devoid of information relating to the extraction of symbol sync from multilevel baseband data. With the recent interest in multilevel amplitude-shift-keying (MASK) and quadrature amplitude-shift-keying (QASK) as signaling techniques for multilevel digital communications systems, the problem of providing symbol synchronization in the receivers of such systems becomes paramount.

This paper presents a technique for extracting symbol sync from an MASK or QASK signal. The scheme is essentially a generalization of the data transition tracking loop (DTTL) which has heretofore been used in PSK systems. The performance of the loop is analyzed in terms of its mean-squared symbol sync jitter and its effects on the data detection process in MASK and QASK systems.
\end{abstract}




\section{DATA-DERIVED SYMBOL SYNCHRONIZATION \\ OF MASK AND QASK SIGNALS}

\section{A. INTRODUCTION.}

Much has been said in the literature ${ }^{[1]-[15]}$ with regard to the problem of establishing symbol synchronization in binary baseband digital communication systems. By comparison, the literature is virtually devoid of information relating to the extraction of symbol sync from multilevel baseband data. With the recent interest ${ }^{[16]-[27]}$ in multilevel amplitude-shift-keying (MASK) and quadrature amplitude-shift-keying (QASK) as signaling techniques for multilevel digital communication systems, the problem of providing symbol synchronization in the receivers of such systems becomes paramount.

This paper presents a technique for extracting symbol sync from an MASK or QASK signal. The scheme is essentially a generalization of the data transition tracking loop (DTTL) ${ }^{[1],[8]-[12]}$ which has heretofore been used in PSK systems. The performance of the loop is analyzed in terms of its mean-squared symbol sync jitter and its effects on the data detection process in MASK and QASK systems.

\section{B. DESCRIPTION OF LOOP OPERATION.}

Consider the symbol synchronizer illustrated in Fig. 1 which is a generalization of the DTTL to the case of an MASK input signal. The basic principle of operation of this loop is virtually no different from its binary counterpart [1], [8]-[12] in that the upper (in-phase) branch monitors the transitions in the input data while the lower (mid-phase) branch produces a measure of the lack of synchronization between the input signal and the locally derived timing source. The essential differences between the two loops are: (a) the hard limiter of the DTTL is replaced by a multilevel quantizer to accommodate the multilevel signal input and (b) an additional feed-forward path is provided to cancel out the bias voltage introduced by the mid-phase branch even in the absence of any synchronization error. Such a bias is not produced in the binary DTTL since, in the absence of noise and synchronization error, there are only two possible signal levels across a data transition and the se levels are equal and opposite. 
The input signal $s(t, \epsilon)$ is a random pulse train representing the data symbols, i.e.

$$
s(t, \epsilon)=\sum_{k} a_{k} p[t-k T-\epsilon]
$$

where $p(t)$ is the unit rectangular pulse defined by $p(t)=1,0 \leq t \leq T, p(t)=0$; all other $T, T$ is the symbol period, $a_{k}$, the symbol amplitude, takes on equally likely values is with $i= \pm 1, \pm 3, \cdots \pm(K-1)$, and $\epsilon$ is the random epoch (assumed to be uniformly distributed) to be continuously estimated. The input additive noise process, $\mathrm{n}(\mathrm{t})$, is assumed to be white Gaussian with a two-sided spectral density, $\mathrm{N}_{\mathrm{o}} / 2$. The sum, $\mathrm{y}(\mathrm{t})$, of signal plus noise is input to in-phase and midphase filters, so-called because they operate, respectively, across a given symbol and between a pair of adjacent symbols. These filters are, in principle, matched filters to the input (i.e. integrate and dump circuits), with the interval of integration being determined by the estimate $\hat{\epsilon}$ of epoch produced by the symbol sync loop. The output $y_{1}(t)$ of the in-phase filter is sampled at intervals of $T$ sec. These sample values $\left\{y_{1 k}\right\}$ are input to a decision device and a decision is made corresponding to each raultilevel input data symbol. The decision device is a multilevel quantizer whose characteristic is given by

$$
Q_{K}(x)= \begin{cases}i \delta, & \text { for }(i-1) \delta \leq x<(i+1) \delta ; \\ (K-1) \delta, & \text { for }(K-2) \delta \leq x<\infty \\ -(K-1) \delta, & \text { for }-\infty \leq x<-(K-2) \delta .\end{cases}
$$

A correct decision

$$
\hat{a}_{k-1} \triangleq Q_{K}\left\{y_{1 k}\right\}
$$

is made whenever $\hat{a}_{k-1}=a_{k-1}$ corresponding to the transmitted symbol in that particular symbol interval. The transition detector then examines two adjacent decision $\hat{a}_{k-1}$ and $\hat{a}_{k}$ and records an output whenever there is a transition in the data, i.e. 


$$
I_{k}=\frac{\hat{a}_{k-1}-\hat{a}_{k}}{2}
$$

We note from (2) and (3) that $I_{k}$ ranges over the set of allowable values $0, \pm \delta, \pm 2 \delta, \cdots, \pm(\mathrm{K}-1) \delta$. Thus, the output of the transition detector is both a measure of the presence and the magnitude of a data symbol transition.

The output, $y_{2}(t)$, of the mid-phase filter is also sampled at intervals of $T$ sec. These sample values $\left\{\mathrm{y}_{2 \mathrm{k}}\right\}$ must be delayed an amount $T=(1-\xi / 2) \mathrm{T}$ sec. before multiplication with the appropriate transition detector output $I_{k}$. The quantity $\xi T, 0 \leq \xi \leq 1$, is the integration interval of the mid-phase integrateand-dump and is often referred to as its window width. For cases of practical interest, where the ratio of two-sided loop bandwidth $W_{L}$ (to be evaluated later on) to data symbol rate $R=1 / T$ is much less than one, the presence of the delay $T$ in the loop has negligible effect on its steady-state noise performance. We shall denote the output of the delay element by $\mathrm{J}_{\mathrm{k}}$ corresponding to the input $\mathrm{y}_{2 \mathrm{k}^{*}}$

To understand the necessity for the additional feed-forward arm in Fig. 1, we examine the sampled output, $y_{2 k}$, of the mid-phase filter in the absence of additive input noise. If we define the normalized synchronization error by $\lambda \triangleq(\epsilon-\hat{\epsilon}) / T$, then we can show that

$$
\mathrm{J}_{k}=\left(\frac{\xi}{2}+\lambda\right) a_{k-1}+\left(\frac{\xi}{2}-\lambda\right) a_{k}=\left(a_{k-1}+a_{k}\right) \frac{\xi}{2}-\left(a_{k}-a_{k-1}\right) \lambda
$$

For the case of binary data, i.e., $a_{k}= \pm \delta$, the presence of a data transition results in either $J_{k}=2 \lambda \delta$ or $J_{k} \doteq-2 \lambda \delta$ depending upon the direction of the transition. Furthermore, if the sync estimation is perfect $(\lambda=0)$, then $J_{k}=0$. For the case of multilevel data, we note that even for the case of perfect sync, a mid-phase filter output voltage still exists, namely $\mathrm{J}_{k}=\left(a_{k-1}+a_{k}\right) \xi / 2$. Thus, in an attempt to remove this bias from the system, we subtract from $J_{k}$ the bias voltage

$$
I_{k} \triangleq\left(\hat{a}_{k-1}+\hat{a}_{k}\right) \frac{\xi}{2}
$$


The net output of this operation, i.e. $J_{k}-I_{k}^{\prime}$ is then multiplied by $I_{k}$ to produce the error signal $e_{k}$ : This error signal is operated upon by a digital filter whose output is used to control a timing pulse generator. An estimate, $\hat{\epsilon}$, of the input random epoch is thus formed every $T$ sec.

In the next section, we consider the steady-state tracking performance of the loop in terms of the variance of its timing error (the so-called timing jitter). The system parameters which characterize such performance are: (1) loop bandwidth-symbol time product $W_{L} T$, (2) two-sided noise spectral density, $\mathrm{N}_{0} / 2$, of the input additive noise, and (3) average signal power $\mathrm{S}$ where

$$
S=\delta^{2}\left(K^{2}-1\right) / 3
$$

\section{SYSTEM ANALYSIS}

We begin with the task of representing our symbol tracker as an equivalent phase-locked loop whose steady-state performance can be found from a well-developed theory (i.e. application of the Fokker-Planck equation). The assumptions under which such a representation is valid will be given below. Developing the above equivalence relies on finding (a) the average loop $\mathrm{S}$-curve, $g(\lambda)$, as a function of the normalized phase error $\lambda=(\epsilon-\hat{\epsilon}) / T$, and (b) the two-sided spectral density about the origin, $S(\omega, \lambda)$, of the equivalent additive noise, $n_{\lambda}(t)$, at the output of the loop nonlinearity, $g(\lambda)$. Once having determined these quantities, the transition tracking loop may be represented as shown in Fig. 2.

The approach taken in finding $g(\lambda)$ and $S(\omega, \lambda)$ follows that of Refs. [7], [10]. The assumptions which must be imposed on the system are as follows:

(A) The input process, $\epsilon(t)$, is essentially constant over a large number of symbol intervals.

(B) $\hat{\epsilon}(t)$, the estimate of $\epsilon(t)$, is essentially constant over a large number of symbol intervals. This fact is a direct consequence of a previous assumption that the response of the loop is very slow with respect to a symbol interval (i.e. $W_{L} T \ll 1$ ). 
It follows from assumptions (A) and (B) that the loop can be analyzed as a continuous loop, and that the statistics of the $r$ andom variable $e_{k}$ (see Fig. I) can be determined assuming $\lambda$ fixed. In effect, then, what we do is to consider many records of the discrete $r . v, e_{k}$ at fixed $\lambda$ and call the average value of this ensemble, $g(\lambda)$, and its spectrum $S(\omega, \lambda)$.

Referring to Fig. 1 , the output of the in-phase integrate-and-dump circuit is

$$
y_{1}(k T+\hat{\epsilon}) \triangleq y_{1 k}=c_{k}+v_{k}
$$

where

$$
\begin{aligned}
& c_{k}=\frac{1}{T} \int_{(k-1) T+\hat{\epsilon}}^{k T+\hat{\epsilon}} s(t, \epsilon) d t \\
& v_{k}=\frac{1}{T} \int_{(k-1) T+\hat{\epsilon}}^{k T+\hat{\epsilon}} n(t) d t
\end{aligned}
$$

The output of the mid-phase integrate-and-dump circuit is

$$
y_{2}\left[\left(k+\frac{\xi}{2}\right) T+\hat{\epsilon}\right] \triangleq y_{2 k}=b_{k}+\mu_{k}
$$

where

$$
\begin{aligned}
& b_{k}=\frac{1}{T} \int_{(k-\xi / 2) T+\hat{\epsilon}}^{(k+\xi / 2) T+\hat{\epsilon}} s(t, \epsilon) d t \\
& \mu_{k}=\frac{1}{T} \int_{(k-\xi / 2) T+\hat{\epsilon}}^{(k+\xi / 2) T+\hat{\epsilon}} n(t) d t
\end{aligned}
$$


Since $\mu_{k}$ and $v_{k}$ are not independent, we wish to express them in terms of a new set of random variables:

$$
\begin{aligned}
& v_{k}=N_{k}+M_{k} \\
& \mu_{k}=N_{k-1}^{\prime}+M_{k}^{\prime}
\end{aligned}
$$

where

$$
\begin{aligned}
& N_{k}=\frac{1}{T} \int_{(k-1) T+\hat{\epsilon}}^{(k-1 / 2) T+\hat{\epsilon}} n(t) d t \quad ; \quad M_{k}=\frac{1}{T} \int_{(k-1 / 2) T+\hat{\epsilon}}^{k T+\hat{\epsilon}} n(t) d t \\
& N_{k}^{\prime}=\frac{1}{T} \int_{(k-1) T+\hat{\epsilon}}^{(k-1+\xi / 2) T+\hat{\epsilon}} n(t) d t \quad ; \quad M_{k}^{\prime}=\frac{1}{T} \int_{(k-\xi / 2) T+\hat{\epsilon}}^{k T+\hat{\epsilon}} n(t) d t
\end{aligned}
$$

with the properties

$\mathrm{N}_{\mathrm{k}}, \mathrm{M}_{\mathrm{n}}$ are mutually independent for all $\mathrm{k}, \mathrm{n}$

$N_{k}^{\prime}, M_{n}^{\prime}$ are mutually independent for all $k, n$

$N_{k}^{\prime}, M_{n}$ and $M_{k}^{\prime}, N_{n}$ are mutually independent for all $k, n$.

$N_{k}, N_{n}^{\prime}$ and $M_{k}, M_{n}^{\prime}$ are mutually independent for all $k \neq n$.

All $\mathrm{M}_{\mathrm{k}}, \mathrm{M}_{\mathrm{k}}^{\prime}, \mathrm{N}_{\mathrm{k}}, \mathrm{N}_{\mathrm{k}}^{\prime}$ and their sums are Gaussian random variables with zero mean and variances,

$$
\sigma_{N_{k}}^{2}=\sigma_{M_{k}}^{2} \triangleq \sigma^{2}=\frac{N_{0}}{4 T} \quad ; \quad \sigma_{M_{k}^{\prime}}^{2}=\sigma_{N_{k}^{\prime}}^{2} \triangleq \sigma^{\prime 2}=\xi \frac{N_{0}}{4 T} \text { for all } k
$$

Recalling that $\mathrm{y}_{2 \mathrm{k}}=\mathrm{J}_{\mathrm{k}}$ (if the mid-phase channel delay is ignored), and $e_{k}=I_{k}\left[J_{k}-I_{k}^{l}\right]$, then combining (1) - (4) and (6) - (14) we get 


$$
\begin{aligned}
e_{k}= & \left\{b_{k}+M_{k}^{\prime}+N_{k+1}^{\prime}-\xi\left[\frac{Q_{K}\left(c_{k}+M_{k}+N_{k}\right)+Q_{K}\left(c_{k+1}+M_{k+1}+N_{k+1}\right)}{2}\right]\right\} \\
& \times\left\{\frac{Q_{K}\left(c_{k}+M_{k}+N_{k}\right)-Q_{K}\left(c_{k+1}+M_{k+1}+N_{k+1}\right)}{2}\right\}
\end{aligned}
$$

As previously mentioned, we define the loop nonlinearity, $g(\lambda)$, by

$$
g(\lambda)=E_{n, s}\left\{e_{k} \mid \lambda\right\}
$$

where $E_{n, s}$ represents the conditional expectation on $\lambda$ both with respect to the noise and signal (symbol sequence). The evaluation of $g(\lambda)$ (for positive $\lambda$ ) is done in Appendix A, with the result ${ }^{*}$

$$
g(\lambda)=\left\{\begin{array}{c}
\frac{3 S}{2\left(\mathrm{~K}^{2}-1\right) \mathrm{K}^{2}} \sum_{\mathrm{m}, \mathrm{j}, \ell} \epsilon_{\ell}\left\{\left[\mathrm{m}(2 \lambda)-j\left(\frac{\xi}{2}+\lambda\right)\right][\operatorname{erf}(\mathrm{j}, \mathrm{m}, \ell)-\operatorname{erf}(-j,-\mathrm{m}, \ell)]\right. \\
\left.-\left[\mathrm{m}(2 \lambda)+j\left(\frac{\xi}{2}+\lambda\right)\right][\operatorname{erf}(j,-m, \ell)-\operatorname{erf}(-j, m, \ell)]\right\} \\
0 \leq \lambda \leq \xi / 2 \\
\frac{3 S \xi}{2\left(\mathrm{~K}^{2}-1\right) \mathrm{K}^{2}} \sum_{m, j, \ell} \epsilon_{\ell}\{(\mathrm{m}-j)[\operatorname{erf}(j, m, \ell)-\operatorname{erf}(-j,-m, \ell)] \\
-(m+j)[\operatorname{erf}(j,-m, \ell)-\operatorname{erf}(-j, m, \ell)]\}
\end{array}\right.
$$

\footnotetext{
*Unless otherwise noted, here and in the remainder of the text, sums over $m$ \& $\mathrm{j}$ are for values $m, j=1,3,5, \cdots,(\mathrm{K}-1)$, while the sum over $\ell$ is for value $\mathrm{s}$ $\ell=0,2,4, \cdots,(K-2)$. It is also easy to show that $g(\lambda)$ is an odd function of $\lambda$.
} 
where $\epsilon_{\ell}$ is the Neumann number defined by $\epsilon_{0}=1, \epsilon_{k}=2 ; \mathrm{k} \geq 1$, and we have introduced the shorthand notation

$$
\operatorname{erf}(j, m, l) \triangleq \operatorname{erf}\left[\sqrt{R_{d}^{\prime}}(j \lambda+m(1-\lambda)+\ell)\right]
$$

with

$$
\begin{aligned}
& R_{d} \triangleq \frac{S T}{N_{0}} \\
& R_{d}^{\prime} \triangleq \frac{R_{d}}{\left(K^{2}-1\right) / 3} \\
& \operatorname{erf}(x) \triangleq \frac{2}{\sqrt{\pi}} \int_{0}^{x} \exp \left(-t^{2}\right) d t
\end{aligned}
$$

It is interesting to note that in arriving at (16), the same result would be obtained if the feed-forward signal had been completely eliminated. The conclusion to be drawn from this is that, on the average, the loop $\mathrm{S}$-curve is unaffected by the bias previously discussed. Keeping in mind, however, that the actual loop operates discretely in that the error signal $e_{k}$ is updated every $T$ sec, then the presence of the feed-forward signal $I_{k}^{\prime}$ is quite important in reducing the instantaneous variations of $\mathrm{e}_{\mathrm{k}}$ about its mean.

Figures 3 and 4 illustrate the normalized S-curve $g_{n}(\lambda) \triangleq g(\lambda) / \mathrm{s}$, defined above, with $R_{d}$ as a parameter, for $K=4$ and window widths $\xi=1$ and $\xi=0.5$ respectively. The limiting $S$-curve for $R_{d} \rightarrow \infty$ is also indicated on these figures.

Now we examine the problem of finding $S(\omega, \lambda)$, the spectrum of the additive noise $n_{\lambda}(t) \triangleq e_{k}(t)-g(\lambda)$, defined by

$$
\begin{aligned}
\mathrm{S}(\omega, \lambda) & =\mathscr{F}\left\{\mathrm{E}_{\mathrm{n}, \mathrm{s}}\left(\mathrm{e}_{\mathrm{k}} \mathrm{e}_{\mathrm{k}+\mathrm{m}} \mid \lambda\right)-\mathrm{g}^{2}(\lambda)\right\} \\
& =\mathscr{F}\{\mathrm{R}(\mathrm{m}, \lambda)\}
\end{aligned}
$$


The symbol denotes the discrete Fourier Transform, i.e.

$$
S(\omega, \lambda)=\sum_{m=-\infty}^{\infty} R(m, \lambda) \cos \omega m T
$$

As for the value of the spectrum as a function of frequency, we note that $R(m, \lambda)$ has non-zero value only at $\mathrm{m}=0, \pm 1$. Thus, the spectrum consists of the sum of a constant and a sinusoidal component with period $\omega_{0}=2 \pi / T$. Since as before $W_{L} T \ll 1$ is assumed, it is sufficient then to consider only the value of zero frequency, i.e. $S(0, \lambda)$ and as sume a flat spectrum of this value for all $\omega$ of interest. Also, since in a practical system little data degradation due to symbol sync error can be tolerated, it is realistic to approximate $S(0, \lambda)$ by its value at $\lambda=0$, viz., $S(0,0)=R(0,0)+2 R(1,0)$. Such approximations were made for the binary data symbol sync loop described in Refs. [7], [10] and were shown there to result in negligible performance penalties for system parameters of practical interest. The computation of $S(0,0)$ is a tedious task and is presented in Appendix B. The result is ${ }^{*}$

$$
\begin{aligned}
h(0)=\frac{S(0,0)}{\left(\frac{N_{0}}{2 T}\right)^{2} \xi R_{d}} \triangleq & \frac{3(K-1)^{2}}{K^{2}-1}+\frac{(K-1)^{2}}{2} \xi R_{d}^{\prime} \\
& \left.-\frac{6}{K^{2}\left(K^{2}-1\right)} \xi\left[\sqrt{R_{d}^{\prime}} \sum_{m} m \sum_{\ell} \operatorname{erf}\left[(m-\ell) \sqrt{R_{d}^{\prime}}\right]+\frac{\sum_{m} \sum_{\ell} \exp \left[-(m-\ell)^{2} R_{d}^{\prime}\right]}{\sqrt{\pi}}\right]\right]^{2} \\
& +\frac{6}{K\left(K^{2}-1\right)}\left[2+\xi R_{d}^{\prime}\left(\frac{K^{2}-1}{3}\right)\right] \sum_{m} \sum_{\ell} \ell \operatorname{erf}\left[(m-\ell) \sqrt{R_{d}^{\prime}}\right]
\end{aligned}
$$

*Here the sum over $\ell$ is for values $\ell=0, \pm 2, \pm 4, \ldots, \pm(\mathrm{K}-2)$. 
The normalization in (21) is chosen so that

$$
\lim _{\mathrm{d}_{\mathrm{d}}} \mathrm{h}(0)=1
$$

Also, for $\mathrm{K}=2, \mathrm{Eq}$. (21) reduces to the previously reported result [7]

$$
h(0)=1+\frac{\xi R_{d}}{2}-\frac{\xi}{2}\left[\sqrt{R_{d}} \text { erf } \sqrt{R_{d}}+\frac{\exp \left(-R_{d}\right)}{\sqrt{\pi}}\right]^{2}
$$

Finally, we note that as in the case of the S-curve calculation, the evaluation of the expression for $h(0)$ given in (21) is independent of the presence of the feedforward signal; i.e. the terms involving this signal when averaged over the noise and symbol sequence result in zero net contribution. Figure 5, 6 and 7 illustrate the normalized noise spectrum $h(0)$ vs. signal-to-noise ratio $R_{d}$ with window width $\xi$ as a parameter and $\mathrm{K}=2,4,8$ respectively.

\section{APPLICATION OF THE FOKKER-PLANCK EQUATION}

The stochastic differential equation which describes the loop illustrated in Fig. 2 is

$$
\dot{\lambda}=-\mathrm{K}_{\mathrm{v}} F(\mathrm{p})\left[\mathrm{g}(\lambda)+\mathrm{n}_{\lambda}(\mathrm{t})\right]
$$

where $\mathrm{p}$ is the differential operation $\mathrm{d} / \mathrm{dt}$ and $\mathrm{K}_{\mathrm{V}}$ is the gain of the timing pulse generator. In writing the above, we have assumed zero static timing error. This is consistent with our previous as sumption that $\dot{\epsilon}(t) \cong 0$.

When dealing with equations of the above form, our aim is to find the steady-state probability density function (p.d.f.) of $\lambda, p(\lambda)$, from which the mean-squared timing error, $\sigma_{\lambda}^{2}$, can be calculated. If we assume that $n_{\lambda}(t)$ is approximately a Gaussian process, then the solution for $p(\lambda)$ can be found via the Fokker-Planck method. The validity of this assumption and the manner in which the method is applied are discussed in Refs. [7], [10]. For simplicity we will discuss only the first order case (i.e. $F(p)=K_{p}$ ) whereupon the solution is of the form 


$$
p(\lambda)=c_{1} \exp \left\{-\frac{2 R d^{\delta} d^{K} g}{\xi h(0)} \int_{0}^{\lambda} g_{n}(y) d y\right\} ; \quad|\lambda| \leq \frac{1}{2}
$$

where

$$
\begin{aligned}
& \delta_{\mathrm{d}}=\frac{2}{\mathrm{~W}_{\mathrm{L}}^{\mathrm{T}}} \\
& W_{L}=\frac{\sqrt{S} K_{v} K_{p} K_{g}}{2}
\end{aligned}
$$

with $W_{L}$ denoting the two-sided loop bandwidth and $K_{g}$ the slope of $g_{n}(\lambda)$ at $\lambda=0$; that is (see Appendix C)

$$
\begin{aligned}
K_{g}=\frac{3 K}{2\left(K^{2}-1\right)}\{ & \sum_{n=-K+3,-K+5, \ldots,-1} \frac{(n+K-1)^{2}}{K^{2}} \operatorname{erf}\left(n \sqrt{R_{d}^{\prime}}\right)+\sum_{n=1,3, \cdots, K-1} \operatorname{erf}\left(n \sqrt{R_{d}^{\prime}}\right) \\
& \left.+\sum_{n=K+1, K+3, \cdots, 2 K-3}\left[1-\frac{(n-K+1)^{2}}{K^{2}}\right] \operatorname{erf}\left(n \sqrt{R_{d}^{\prime}}\right)\right\} \\
& -\frac{\xi}{2} \sqrt{\frac{R_{d}^{\prime}}{\pi}}\left\{\sum_{n=-K+3,-K+5, \cdots,-1}\left[1+\frac{n-1}{\bar{K}}\right] \exp \left(-n^{2} R_{d}^{\prime}\right)\right. \\
& +\sum_{n=1,3, \cdots, K-1} \exp \left(-n^{2} R_{d}^{\prime}\right) \\
& \left.+\sum_{n=K+1, K+3, \cdots, 2 K-3}\left[1-\frac{n-(K-1)}{K}\right] \exp \left(-n^{2} R_{d}^{\prime}\right)\right\}
\end{aligned}
$$


Note that

$$
\lim _{d \rightarrow \infty} K_{g}=1
$$

Plotted in Figs. 8, 9, and 10 are $K_{g}$ as given by (27) vs. $R_{d}$ with $\xi$ as a parameter and $K=2,4,8$ respectively. The normalized mean-squared sync error $\sigma_{\lambda}^{2}$ is then given by

$$
\sigma_{\lambda}^{2}=\int_{-1 / 2}^{1 / 2} \lambda^{2} p(\lambda) d \lambda
$$

For large $\mathrm{R}_{\mathrm{d}}{ }^{\delta} \mathrm{d}$ (the case of practical interest), we can approximate $g_{n}(\lambda)$ by $K_{g} \lambda$ in Eq. (25). Making this substitution gives

$$
\mathrm{p}(\lambda) \cong \mathrm{C}_{1} \exp \left\{-\frac{2 \mathrm{R} \mathrm{d}^{\delta} \mathrm{d}^{2} \mathrm{~g}}{\xi \mathrm{h}(0)}\left(\frac{\lambda^{2}}{2}\right)\right\} \quad ; \quad|\lambda| \leq \frac{1}{2}
$$

Furthermore, since $p(\lambda)$ now has significant values only on a small interval of $\lambda$ relative to one-half, then the finite integral in (29) can be approximated by an integral from zero to infinity. Thus, by inspection of $(30)$ we have that for large $R_{d}^{\delta}$,

$$
\sigma_{\lambda}^{2} \cong \frac{\xi h(0)}{2 R_{d} \delta \mathrm{d}^{2}}
$$

If now in addition $R_{d}$ is large, then from (22), (28), and (31) we have asymptotically that

$$
\sigma_{\lambda}^{2} \cong \frac{\xi}{2 R_{d^{\delta} \mathrm{d}}}
$$


which is independent of $K$ and hence is the identical result as that obtained for the binary symbol sync loop [1], [7], [10]. Plotted in Figs. 11, 12, and 13 is the rms symbol sync jitter $\sigma_{\lambda}$ [as computed from (31)] vs $R_{d}$ with $\delta_{d}$ as a parameter, $\xi=1$, and $K=2,4,8$ respectively. We see from these results that the performance of the synchronizer (insofar as this measure is concerned) is virtually independent of the number of signal levels $\mathrm{K}$.

\section{E. ERROR PROBABILITY PERFORMANCE}

This section deals with the effect of symbol sync error on system error probability performance. Since the optimum detector for an MASK signal set is a matched filter followed by a $\mathrm{K}$-level quantizer, we are interested primarily in examining the degradation in error probability performance of this detector when the symbol synchronization reference is noisy. The first step in the presentation will be to arrive at the error probability of the MASK detector conditioned on a symbol sync error. This conditional error probability can then be averaged over the p.d.f. $p(\lambda)$ of (25) or (30) to yield the average error probability performance of the receiver. When compared with a source of perfect symbol sync, the degradation in performance due to symbol sync error can be assessed.

Consider the situation where the symbol sync is in error by $\epsilon=\lambda T$ sec. The matched filter statistic on which a decision is made for the nth transmitted symbol now depends on the received data in the time interval (n - 1) $\mathrm{T}+\hat{\epsilon} \leq t$ $\leq \mathrm{nT}+\hat{\epsilon}$ where $\hat{\epsilon}$ is the sync estimate obtained from the symbol synchronizer. Stated mathematically,

$$
\hat{a}_{n}=Q_{K}\left\{\frac{1}{T} \int_{(n-1) T+\hat{\epsilon}}^{n T+\hat{\epsilon}} y(t) p[t-(n-1) T-\hat{\epsilon}] d t\right\}
$$

where $Q_{K}(x)$ is the $K$-level quantizer characteristic defined in (7), and $y(t)=s(t, \epsilon)+n(t)$ is the matched filter input. Following an approach analogous to that given in [1] for binary data, we arrive at an expression for the conditional error probability $\mathrm{P}_{\mathrm{E}}(\lambda)$ of an MASK input (see Appendix D), that is 


$$
P_{E}(\lambda)=\frac{1}{K^{2}} \sum_{j= \pm 1, \pm 3, \cdots, \pm(K-1)} \sum_{\ell=0, \pm 2, \pm 4, \cdots, \pm(K-2)} \operatorname{erfc}\left\{\sqrt{R_{d}^{\prime}}[\ell-(\ell-1)(1-|\lambda|)-j|\lambda|]\right\}
$$

For $K=2$, (34) reduces to the well-known result for NRZ data

$$
\mathrm{P}_{E}(\lambda)=\frac{1}{4} \operatorname{erfc}\left(\sqrt{\mathrm{R}_{\mathrm{d}}}\right)+\frac{1}{4} \operatorname{erfc}\left[\sqrt{\mathrm{R}_{\mathrm{d}}}(1-4|\lambda|)\right]
$$

where

$$
\operatorname{erfc}(x) \triangleq 1-\operatorname{erf}(x)
$$

The average error probability $P_{E}$ is determined from

$$
P_{E}=\int_{-1 / 2}^{1 / 2} P_{E}(\lambda) p(\lambda) d \lambda
$$

Assuming large $R_{d} \delta_{d}$, then substituting (30) and (34) into (37) and performing numerical integration on a digital computer provides the graphical results of Figs. 14, 15. In these figures the average error probability is plotted versus $\mathrm{R}_{\mathrm{d}}$ with $\delta_{-\mathrm{d}}$ as a parameter for $\xi=1$ and $\mathrm{K}=2,4$ respectively. We see from these figures that $a \delta_{d}$ of 250 (for $K=2$ ) and $a \delta_{d}$ of 100 (for $K=4$ ) are sufficient to reduce the signal-to-noise ratio degradation (relative to ideal performance) to around $0.1 \mathrm{~dB}$. In a practical system, $\delta_{\mathrm{d}}$ would be more of the order of thousands.

F. MODIFICATION OF THE MASK SYMBOL SYNC LOOP FOR A QASK INPUT

In a QASK system, one has available two independent MASK signals as inputs to the symbol synchronizer. In particular, assuming perfect carrier synchronization, the output of the in-phase and quadrature carrier demodulators of the QASK receiver can be written, respectively, in the form 


$$
\begin{aligned}
y_{I}(t) & =s_{I}(t, \epsilon)+n_{I}(t) \\
y_{Q}(t) & =s_{Q}(t, \epsilon)+n_{Q}(t)
\end{aligned}
$$

where

$$
\begin{aligned}
s_{I}(t, \epsilon) & =\sum_{n} a_{n} p[t-n T-\epsilon] \\
s_{Q}(t, \epsilon) & =\sum_{n} b_{n} p[t-n T-\epsilon]
\end{aligned}
$$

Here $p(t)$ is again the unit rectangular pulse defined by $p(t)=1,0 \leq t \leq T$, $p(t)=0$; all other $T$, and the quadrature amplitudes $a_{i}$ and $b_{j}$ independently take on equally likely values $i \delta$ and $j \delta$ with $i, j= \pm 1, \pm 3, \cdots, \pm(K-1)$. Also $n_{I}(t)$ and ${ }^{n}{ }_{Q}(t)$ are uncorrelated (independent) white Gaussian noise processes with twosided spectral density $\mathrm{N}_{\mathrm{o}} / 2$. If the carrier synchronization is not perfect, then the carrier demodulator outputs are respectively given by [25, Eq. 7].

It is a simple matter then to modify the MASK symbol synchronizer of Fig. 1 to accommodate a QASK input. Fig. 16 illustrates such an implementation. Basically, each input of (38) is fed through identical in-phase and quadrature circuitry with the resultant error signals added to produce the required symbol sync estimate. Thus, it appears at first glance that for the same signal step size $\delta$, the QASK symbol sync loop has an S-curve whose values are twice that of the MASK loop. However, one must remember that the average transmitted power of a QASK signal set is $S=\left[2\left(K^{2}-1\right) / 3\right] \delta^{2}$, i. e., twice that of an MASK set. Thus, for equal average transmitted powers, the p.d.f. of $\lambda$ for the QASK $-K^{2}$ symbol sync loop of Fig. 16 is identical to that of the K-level MASK loop of Fig. 1. In the above, the notation QASK $-K^{2}$ denotes a QASK system with $\mathrm{K}^{2}(\mathrm{~K} \times \mathrm{K})$ signals. Finally, one can relate the conditional error probability of a QASK $-K^{2}$ system to that of a K-level MASK system by

$$
\left.P_{E}(\lambda)\right|_{\text {QASK }}=\left.2 P_{E}(\lambda)\right|_{\text {MASK }}\left[1-\left.\frac{1}{2} P_{E}(\lambda)\right|_{\text {MASK }}\right]
$$


where $\left.P_{E}(\lambda)\right|_{\text {MASK }}$ is given by Eq. (34) with $R_{d}^{1}$ replaced by $R_{d}^{\prime} / 2$. Figs. 17,18 illustrate the average error probability performance of a QASK system obtained by substituting (38) and (40) into (37) and performing numerical integration on a digital computer. As in the MASK case, the average error probability is plotted vs $R_{d}$ with $\delta_{d}$ as a parameter for $\xi=1$ and $\mathrm{K}^{2}=4,16$ respectively. The case $\mathrm{K}^{2}=4$ corresponds to quadriphase' signaling.

\section{G. CONCLUSIONS}

Summarizing, a symbol synchronizer for MASK and QASK signal sets has been proposed and analyzed insofar as its rms sync jitter performance and the effect of this jitter on system error probability performance. The sync jitter performance has been shown to be virtually independent of the number of signal levels, K. As in the binary case, the jitter performance improves as the window width $\xi T$ (the integration interval in the mid-phase arm) is reduced. The trade-off in reducing the size of the window is that the loop's cycle slip performance degrades. Thus, a compromise value of window width must be chosen. Typically, in the past (for binary systems), a quarter window $(\xi=1 / 4)$ synchronizer has been shown to yield adequate jitter and slip performance. The same conclusion would hold here in the multilevel case. From the results presented on error probability performance, we see that a symbol rate to loop bandwidth ratio $\delta_{\mathrm{d}}=250$ (for $\mathrm{K}=2$ and $\xi=1$ ) and $\mathrm{a} \delta_{\mathrm{d}}=100$ (for $\mathrm{K}=4$ and $\xi=1$ ) are sufficient to reduce the signal-to-noise ratio degradation (relative to the ideal performance) to around .I dB. In a practical system, $\delta_{d}$ would be on the order of thousands and $\xi$ would be less than unity; thus the practical performance of the symbol synchronizer would be virtually ideal.

Although the phase detector characteristics (loop S-curves) obtained for tracking purposes have a rather jagged appearance, it must be remembered that for the system parameters of interest, the loop tracking range would be restricted to a small portion of the $S$-curve in the neighborhood of zero sync error where the $S$-curve can be assumed linear with a slope depending upon signal-to-noise ratio. Also, since these S-curves were obtained by averaging over all possible sequences of three symbols, each symbol ranging over $\mathrm{K}$ levels (the case of interest when tracking the input data), the S-curves for acquisition would be somewhat more regular in shape since there a specific acquisition sequence is transmitted. 


\section{REFERENCES}

1. Lindsey, W. C., and Simon, M. K., Telecommunication Systems Engineering, Chapter 9, Prentice-Hall, Inc., Englewood Cliffs, N.J., 1973.

2. Stiffler, J. J., Theory of Synchronous Communications, Chapter 7, Prentice-Hall, Inc., Englewood Cliff́s, N. J., 1971.

3. Stiffler, J. J., "Maximum Likelihood Symbol Synchronization," Jet Propulsion Laboratory, Pasadena, Calif., SPS 37-35, Vol. IV (October, 1965) 349-357.

4. Sage, A. P., and McBride, A. L., "Optimum Estimation of Bit Synchronization," IEEE Transactions on Aerospace and Electronic Systems, Vol. AES-5, No. 3 (May, 1969) 525-536.

5. Wintz, P.A., and Luecke, E.J., "Performance of Optimum and Suboptimum Synchronizers, "IEEE Transactions on Communication Technology, Vol. COM-17, No. 3 (June, 1969) 380-389.

6. Mengali, U., "A Self Bit Synchronizer Matched to the Signal Shape," IEEE Transactions on Aerospace and Electronic Systems, Vol. AES-7, No. 4 (July, 1971) 686-693.

7. Lindsey, W. C., and Tausworthe, R. C., "Digital Data-Transition Tracking Loops, " Jet Propulsion Laboratory, Pasadena, Calif. SPS 37-50, Vol. III (April, 1968) 272-276.

8. , and Anderson, T. O., "Digital Data-Transition Tracking Loops," Proceedings of the International Telemetering Conference, Los Angeles, Calif. (Oct. 1968) 259-271.

9. Tausworthe, R. C., "Analysis and Design of the Symbol-Tracking Loop," Jet Propulsion Laboratory, Pasadena, Calif., SPS 37-51, Vol. II (May, 1968) $145-147$.

10. Simon, M. K., "An Analysis of the Steady-State Phase Noise Performance of a Digital Data-Transition Tracking Loop," Jet Propulsion Laboratory, Pasadena, Calif. SPS 37-55, Vol. III (February, 1969) 54-62.

11. "Optimization of the Performance of a Digital Data Transition Tracking Loop," IEEE Transactions on Communication Technology, Vol. COM-18, No. 3 (October, 1970) 686-690.

12. Hurd, W.J., and Anderson, T.O., "Digital Transition Tracking Symbol Synchronizer for low SNR Coded Systems," IEEE Transactions on Communication Technology, Vol. COM-18, No. 2 (April, 1970) 141-147.

13. Simon, M. K., "Nonlinear Analysis of an Absolute Value Type of EarlyLate-Gate Bit Synchronizer, "IEEE Transactions on Communication Technology, Vol. COM-18, No. 3 (October, 1970) 589-596. 
14. Layland, J. W., "Telemetry Bit Synchronization Loop," Jet Propulsion Laboratory, Pasadena, Calif. SPS 34-46, Vol. III (July, 1967) 204-215.

15. McRae, D. D. , and Smith, E.F., "Bit Synchronization," Proceedings of the International Telemetering Conference, Los Angeles, Calif. (October, 1972) 539-552.

16. J. Salz, J.R. Sheehan, and D. J. Paris, "Data Transmission by Combined AM and PM," Bell Syst. Tech. J., Vol. 50, pp. 2399-2419, Sept. 1971.

17. K. Kawai, S. Shintani, and H. Yanagidaira, "Optimum Combination of Amplitude and Phase Modulation Scheme and its Application to Data Transmission Modem," in Conf. Rec., 1972 IEEE Int. Conf. Communications, p. 29-6.

18. C. M. Thomas, "Amplitude-Phase-Keying with M-ary Alphabets: A Technique for Bandwidth Reductions, " in Proc. Int. Telemetering Conf., p. 289, October, 1972.

19. and S. H. Durrani, "Amplitude-Phase Keying: An Evaluation for Realistic Transponder Channels, " presented at the 2nd Conf. Digital Satellite Communication, Paris, France, Nov. 1972.

20. G. R. Welti, "Pulse Amplitude-and-Phase Modulation," presented at the 2nd Conf. Digital Satellite Communications, Paris, France, Nov. 1972.

21. J. G. Smith, "On the Feasibility of Efficient Multi-amplitude Communication," in Nat. Telecommunications Conf. Rec., p. 20E-1, 1972.

22. G. J. Foschini, R. D. Gitlin, and S. B. Weinstein, "On the Selection of a Two-dimensional Signal Constellation in the Presence of Jitter and Gaussian Noise," Bell Syst. Tech. J., Vol. 52, pp. 927-965, July-Aug. 1973.

23. M. K. Simon, and J. G. Smith, "Hexagonal Multiple-Phase-and-Amplitude Shift Keyed Signal Sets, "IEEE Trans. Commun., Vol. COM-21, pp. 1108-1115, October, 1973.

24. G.J. Foschini, R. D. Gitlin, S. B. Weinstein, "Optimization of TwoDimensional Signal Constellations in the Presence of Gaussian Noise," IEEE Transactions on Communications, Vol. COM-22, No. 1, pp. 28-38, January, 1974.

25. J.G. Smith, "A Review of Multiple Amplitude-Phase Digital Signals," IFT Journal, Vol. 1, No. 1, pp. 6-14, Jan/Feb, 1974.

26. M. K. Simon, and J. G. Smith, "Carrier Synchronization and Detection of QASK Signal Sets," IEEE Transactions on Communications, Vol. COM-22, No. 2, pp. 98-106, February, 1974.

27. C. M. Thomas, M. Y. Weidner, and S. H. Durrani, "Digital AmplitudePhase Keying with M-ary Alphabets," IEEE Transactions on Communications, Vol. COM-22, No. 2, 168-180, February, 1974. 


\section{EVALUATION OF THE LOOP NONLINEARITY $g(\lambda)$}

To evaluate $g(\lambda)$ defined in the main text, and considering first the average with respect to the noise, we have*

$$
\begin{aligned}
E_{n}\left\{e_{k}\right\}= & E_{n}\left\{\frac{b_{k}}{2}\left[Q_{K}\left(c_{k}+M_{k}+N_{k}\right)-Q_{K}\left(c_{k+1}+M_{k+1}+N_{k+1}\right)\right]\right\} \\
& +E_{n}\left\{\frac{\left[M_{k}^{\prime}+N_{k+1}^{\prime}\right]}{2}\left[Q_{K}\left(c_{k}+M_{k}+N_{k}\right)-Q_{K}\left(c_{k+1}+M_{k+1}+N_{k+1}\right]\right\}\right. \\
& -E_{n}\left\{\frac{\xi}{4}\left[Q_{K}^{2}\left(c_{k}+M_{k}+N_{k}\right)-Q_{K}^{2}\left(c_{k+1}+M_{k+1}+N_{k+1}\right)\right]\right\}
\end{aligned}
$$

But (see Appendix E)

$$
\begin{aligned}
\mathrm{E}_{\mathrm{n}}\left\{\mathrm{Q}_{\mathrm{K}}(\mathrm{C}+\mathrm{y})\right\} \triangleq & \operatorname{erf}_{\mathrm{K}}\left(\frac{\mathrm{C}}{\sqrt{2 \sigma}}\right) \\
& \triangleq \frac{\delta}{2}\left\{(\mathrm{~K}-1)\left[\operatorname{erf}\left(\frac{\mathrm{C}+(\mathrm{K}-2) \delta}{\sqrt{2} \sigma_{\mathrm{y}}}\right)+\operatorname{erf}\left(\frac{\mathrm{C}-(\mathrm{K}-2) \delta}{\sqrt{2} \sigma_{\mathrm{y}}}\right)\right]\right. \\
& \left.+\sum_{\ell= \pm 1, \pm 3, \cdots, \pm(\mathrm{K}-3)}\left[\operatorname{erf}\left(\frac{C-(\ell-1) \delta}{\sqrt{2} \sigma_{\mathrm{y}}}\right)-\operatorname{erf}\left(\frac{\mathrm{C}-(\ell+1) \delta}{\sqrt{2} \sigma_{\mathrm{y}}}\right)\right]\right\}
\end{aligned}
$$

where

$$
\operatorname{erf} z=\frac{2}{\sqrt{\pi}} \int_{0}^{z} e^{-t^{2}} d t
$$

"Herein we omit the conditioning on $\lambda$ in our notation, assuming it to be understood. 
Thus, the first term in $E_{n}\left\{e_{k}\right\}$ is evaluated as

$$
\frac{b_{k}}{2}\left\{\operatorname{erf}{ }_{K}\left(\frac{c_{k}}{\sqrt{2} \sigma_{\nu}}\right)-\operatorname{erf}{ }_{K}\left(\frac{c_{k+1}}{\sqrt{2} \sigma_{v}}\right)\right\}
$$

where

$$
\sigma_{v}^{2}=\frac{N_{o}}{2 T}=2 \sigma^{2}
$$

It follows from the independence properties of the noise random variables defined in the main text that

$$
\begin{aligned}
& E_{n}\left\{M_{k}^{\prime} Q_{K}\left[c_{k+1}+M_{k+1}+N_{k+1}\right]\right\}=0 \\
& E_{n}\left\{N_{k+1}^{\prime} Q_{K}\left[c_{k}+M_{k}+N_{k}\right]\right\}=0
\end{aligned}
$$

Thus, the second term of $E_{n}\left\{e_{k}\right\}$ reduces to

$$
\frac{1}{2} E_{n}\left\{M_{k}^{\prime} Q_{K}\left[c_{k}+M_{k}+N_{k}\right]-N_{k+1}^{\prime} Q_{K}\left[c_{k+1}+M_{k+1}+N_{k+1}\right]\right\}
$$

In view of $(A-1)$, this reduces to

$$
\begin{aligned}
& \frac{1}{2} E_{n}\left\{M_{k}^{\prime} \operatorname{erf}\left[\frac{c_{k}+M_{k}}{\sqrt{2} \sigma_{N_{k}}}\right]-N_{k+1}^{\prime} \operatorname{erf}\left[\frac{c_{k+1}+N_{k+1}}{\sqrt{2} \sigma_{M_{k+1}}}\right]\right\}= \\
& \frac{1}{2} E_{n}\left\{M_{k}^{\prime} \operatorname{erf}_{K}\left[\frac{c_{k}+M_{k}^{\prime}+M_{k}^{\prime \prime}}{\sqrt{2} \sigma}\right]-N_{k+1}^{\prime} \operatorname{erf}_{K}\left[\frac{c_{k+1}+N_{k+1}^{\prime}+N_{k+1}^{\prime \prime}}{\sqrt{2} \sigma}\right]\right\}
\end{aligned}
$$


where

$$
M_{k}^{\prime \prime}=\int_{(k-1 / 2) T+\hat{\epsilon}}^{(k-\xi / 2) T+\hat{\epsilon}} n(t) d t \quad ; \quad N_{k}^{\prime \prime}=\int_{(k-1+\xi / 2) T+\hat{\epsilon}}^{(k-1 / 2) T+\hat{\epsilon}} n(t) d t \quad(A-7)
$$

Note: $M_{k}^{\prime}, M_{k}^{\prime \prime}$ and $N_{k}^{\prime}, N_{k}^{\prime \prime}$ are mutually independent and

$$
\sigma_{M_{k}^{\prime \prime}}^{\prime 2}=\sigma_{N_{k}^{\prime}}^{\prime 2} \triangleq \sigma^{\prime \prime 2}=\frac{N_{o}}{4 T}(1-\xi)=\sigma^{2}(1-\xi)
$$

It can be shown that,

$$
E\{\operatorname{erf}(a+b x)\}=\operatorname{erf}\left(\frac{a}{\sqrt{1+2 b^{2} \sigma_{x}^{2}}}\right)
$$

Applying $(A-7)-(A-9)$ to $(A-6)$, we get

$$
\frac{1}{2} E\left\{M_{k}^{\prime} \operatorname{erf}\left[\frac{c_{k}+M_{k}^{\prime}}{\sigma \sqrt{2(2-\xi)}}\right]-N_{k+1}^{\prime} \operatorname{erf}\left[\frac{c_{k+1}+N_{k+1}^{\prime}}{\sigma \sqrt{2(2-\xi)}}\right]\right\}
$$

It can also be shown that,

$$
E\{x \operatorname{erf}(a+b x)\}=\frac{2}{\sqrt{\pi}} \frac{\sigma_{x}^{2} b}{\sqrt{1+2 b^{2} \sigma_{x}^{2}}} \exp \left\{\frac{-a^{2}}{1+2 \sigma_{x}^{2} b^{2}}\right\}
$$

Applying $(A-11)$ to $(A-10)$, finally gives

$$
\left(\frac{\sigma^{\prime}}{\sigma}\right) \frac{1}{2 \sqrt{\pi}}\left[\exp _{K}\left(\frac{-c_{k}^{2}}{4 \sigma^{2}}\right)-\exp _{K}\left(\frac{-c_{k+1}^{2}}{4 \sigma^{2}}\right)\right]
$$


or in terms of $\sigma_{v}$,

$$
\xi \frac{\sigma_{\nu}}{2 \sqrt{2 \pi}}\left[\exp \left(\frac{-c_{k}^{2}}{2 \sigma^{2}}\right)-\exp _{K}\left(\frac{-c_{k+1}^{2}}{2 \sigma^{2}}\right)\right]
$$

where (see Appendix E)

$$
\begin{aligned}
\exp _{\mathrm{K}}\left(-\frac{\mathrm{C}^{2}}{2 \sigma_{\mathrm{y}}^{2}}\right) \triangleq & \frac{\delta}{2}\left\{(\mathrm{~K}-1)\left[\exp \left\{-\frac{(\mathrm{C}+(\mathrm{K}-2) \delta)^{2}}{2 \sigma_{\mathrm{y}}^{2}}\right\}+\exp \left\{-\frac{(\mathrm{C}-(\mathrm{K}-2) \delta)^{2}}{2 \sigma_{\mathrm{y}}^{2}}\right\}\right]\right. \\
& +\sum_{\ell= \pm 1, \pm 3, \cdots, \pm(\mathrm{K}-3)}\left[\exp \left\{-\frac{(\mathrm{C}-(\ell-1) \delta)^{2}}{2 \sigma_{\mathrm{y}}^{2}}\right\}\right. \\
& \left.-\exp \left\{-\frac{\left.(\mathrm{C}-(\ell+1) \delta)^{2}\right)}{2 \sigma_{\mathrm{y}}^{2}}\right\}\right]
\end{aligned}
$$

Finally, to evaluate the third term in $E_{n}\left\{e_{k}\right\}$, we define (see Appendix $E$ )

$$
\begin{aligned}
E_{n}\left\{Q_{K}^{2}(C+y)\right\} \triangleq 1_{K}^{(2)}\left(\frac{C}{\sqrt{2} \sigma_{y}}\right) & \\
\triangleq & \frac{\delta^{2}}{2}\left\{(K-1)^{2}\left[2-\operatorname{erf}\left(\frac{C+(K-2) \delta}{\sqrt{2} \sigma_{y}}\right)+\operatorname{erf}\left(\frac{C-(K-2) \delta}{\sqrt{2} \sigma_{y}}\right)\right]\right. \\
& \left.+\sum_{\ell= \pm 1, \pm 3, \cdots, \pm(K-3)} \ell^{2}\left[\operatorname{erf}\left(\frac{C-(\ell-1) \delta}{\sqrt{2} \sigma}\right)-\operatorname{erf}\left(\frac{C-(\ell+1) \delta}{\sqrt{2} \sigma_{y}}\right)\right]\right\}
\end{aligned}
$$

Thus, the third term of $E_{n}\left\{e_{k}\right\}$ is evaluated as 


$$
-\frac{\xi}{4}\left[\mathrm{I}_{\mathrm{K}}^{(2)}\left(\frac{\mathrm{c}_{\mathrm{k}}}{\sqrt{2} \sigma_{\nu}}\right)-\mathrm{I}_{\mathrm{K}}^{(2)}\left(\frac{\mathrm{c}_{\mathrm{k}+1}}{\sqrt{2} \sigma_{\nu}}\right)\right]
$$

Adding (A-2), (A-13), and (A-16)

$$
\begin{aligned}
E_{n}\left\{e_{k}\right\}= & \frac{b_{k}}{2}\left\{\operatorname{erf}\left(\frac{c_{k}}{\sqrt{2} \sigma_{\nu}}\right)-\operatorname{erf}\left(\frac{c_{k+1}}{\sqrt{2} \sigma_{\nu}}\right)\right\} \\
& +\xi \frac{\sigma_{\nu}}{2 \sqrt{2 \pi}}\left[\exp _{K}\left(\frac{-c_{k}^{2}}{2 \sigma_{\nu}^{2}}\right)-\exp _{K}\left(\frac{-c_{k+1}^{2}}{2 \sigma_{\nu}^{2}}\right)\right]-\frac{\xi}{4}\left[1_{K}^{(2)}\left(\frac{c_{k}}{\sqrt{2} \sigma_{\nu}}\right)-1_{K}^{(2)}\left(\frac{c_{k+1}}{\sqrt{2} \sigma_{\nu}}\right)\right]
\end{aligned}
$$

It now remains to average $\mathrm{E}_{\mathrm{n}}\left\{\mathrm{e}_{\mathrm{k}}\right\}$ over the signal distribution. From Eq. (7) of the main text,

$$
\begin{array}{rlr}
\frac{c_{k}}{\delta} & =d_{k-2} \lambda+d_{k-1}(1-\lambda) & \\
\frac{c_{k+1}}{\delta} & =d_{k-1} \lambda+d_{k}(1-\lambda) & \text { for } \lambda \leq \frac{\xi}{2} \\
\frac{b_{k}}{\delta} & =d_{k-1}\left[\frac{\xi}{2}+\lambda\right]+d_{k}\left[\frac{\xi}{2}-\lambda\right]
\end{array}
$$

and

$$
\begin{aligned}
\frac{c_{k}}{\delta} & =d_{k-2} \lambda+d_{k-1}(1-\lambda) \\
\frac{c_{k+1}}{\delta} & =d_{k-1} \lambda+d_{k}(1-\lambda) \quad \text { for } \frac{\xi}{2} \leq \lambda \leq \frac{1}{2} \\
\frac{b_{k}}{\delta} & =d_{k-1} \xi
\end{aligned}
$$


where $d_{k} \triangleq a_{k} / \delta$ takes on values $\pm 1, \pm 3, \cdots, \pm(K-1)$ with equal probability $1 / K$. Substituting $(A-18)$ and $(A-19)$ in $(A-17)$ and averaging overall possible equally likely sequences formed from the symbols $d_{k-2}, d_{k-1}$ and $d_{k}$.

$$
\begin{aligned}
& \int \frac{3 s}{4\left(K^{2}-1\right) K^{3}} \sum_{m, n- \pm 1, \pm 3} \sum_{\ldots, \pm(K-1)}\left[m\left(\frac{\xi}{2}+\lambda\right)+n\left(\frac{\xi}{2}-\lambda\right)\right] \\
& \sum_{j= \pm 1, \pm 3, \ldots \pm \pm(K-1)}\{(K-1)[\operatorname{erf}(j, m, K-2)+\operatorname{erf}(j, m,-(K-2)) \\
& -\operatorname{erf}(m, n, K-2)-\operatorname{erf}(m, n,-(K-2))] \\
& +\sum_{\ell= \pm 1, \pm 3, \cdots, \pm(K-1)} \ell[\operatorname{erf}(j, m,-(\ell-1))-\operatorname{erf}(j, m,-(\ell+1)) \\
& -\operatorname{erf}(\mathrm{m}, \mathrm{n},-(\ell-1))+\operatorname{erf}(\mathrm{m}, \mathrm{n},-(\ell+1))]\} \\
& 0 \leq \lambda \leq \frac{\xi}{2} \\
& g(\lambda)= \\
& \frac{3 S}{4\left(K^{2}-1\right) K^{3}} \sum_{m= \pm 1, \pm 3, \cdots, \pm(K-1)} m \xi \\
& \times \sum_{n, j= \pm 1, \pm 3, \cdots, \pm(K-1)}\{(K-1)[\operatorname{erf}(j, m, K-2)+\operatorname{erf}(j, m,-(K-2)) \\
& -\operatorname{erf}(m, n, K-2)-\operatorname{erf}(m, n,-(K-2))] \\
& +\sum_{\ell= \pm 1, \pm 3, \cdots, \pm(K-1)} \ell[\operatorname{erf}(j, m,-(\ell-1))-\operatorname{erf}(j, m,-(\ell+1)) \\
& -\operatorname{erf}(m, n,-(\ell-1))+\operatorname{erf}\{m, n,-(\ell+1))]\} \\
& \frac{\xi}{2} \leq \lambda \leq \frac{1}{2}
\end{aligned}
$$


where we have introduced the shorthand notation

$$
\operatorname{erf}(j, m, \ell) \triangleq \operatorname{erf}\left[\sqrt{R_{d}^{\prime}}(j \lambda+m(1-\lambda)+\ell)\right]
$$

and

$$
\begin{aligned}
& \mathrm{R}_{\mathrm{d}} \triangleq \frac{\mathrm{ST}}{\mathrm{N}_{0}}=\left(\frac{\mathrm{K}^{2}-1}{3}\right) \frac{\delta^{2}}{2 \sigma_{v}^{2}} \\
& \mathrm{R}_{\mathrm{d}}^{\prime} \triangleq \frac{\mathrm{R}_{\mathrm{d}}}{\left(\frac{\mathrm{K}^{2}-1}{3}\right)}
\end{aligned}
$$

After considerable algebraic manipulation (A-20) may be simplified to

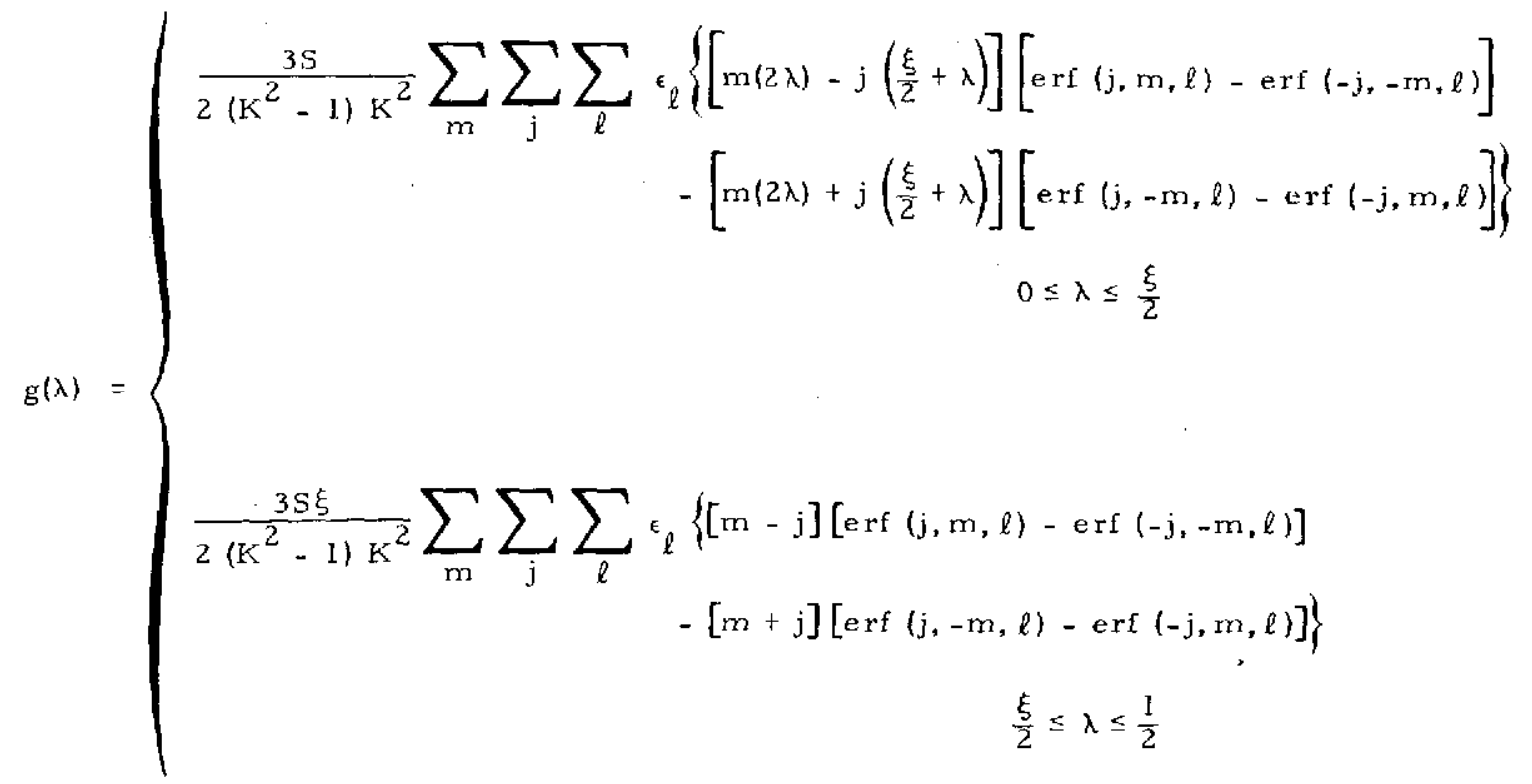

where $\epsilon_{\ell}$ is the Neumann number, i. e., ${ }^{\epsilon_{0}}=1 ;{ }^{{ }}{ }_{\ell}=2, \ell \geq 1$, and the sums on $m$ and $j$ range over the values $1,3, \cdots,(K-1)$ while the sum on $\ell$ ranges over the values $0,2,4, \cdots,(\mathrm{K}-2)$. 


\section{APPENDIX B}

EVALUATION OF THE LOOP SPECTRUM, $S(0,0)$

We begin by considering ${ }^{*}$

$$
\begin{aligned}
E_{n}\left\{e_{k} e_{k+m}\right\}= & E_{n}\left\{\left[b_{k}+M_{k}+N_{k+1}^{\prime}-\xi\left\{\frac{Q_{K}\left(c_{k}+M_{k}+N_{k}\right)+Q_{K}\left(c_{k+1}+M_{k+1}+N_{k+1}\right)}{2}\right\}\right]\right. \\
& \times\left[\frac{Q_{K}\left(c_{k+1}+N_{k+1}+M_{k+1}\right)-Q_{K}\left(c_{k}+N_{k}+M_{k}\right)}{2}\right] \\
& \times\left[b_{k+m}+M_{k+m}^{\prime}+N_{k+m+1}^{\prime}-\xi\left\{\frac{Q_{K}\left(c_{k+m}+M_{k+m}+N_{k+m}\right)+Q_{K}\left(c_{k+1+m}+M_{k+1+m}+N_{k+1+m}\right)}{2}\right\}\right] \\
& \left.\times\left[\frac{Q_{K}\left(c_{k+m+1}+N_{k+m+1}+M_{k+m+1}\right)-Q_{K}\left(c_{k+m}+N_{k+m}+M_{k+m}\right)}{2}\right]\right\}
\end{aligned}
$$

term by term.

TERM NO. 1

$$
\begin{aligned}
\frac{1}{4} E_{n}\left\{b_{k} b_{k+m}\left[Q_{K}\left(c_{k+1}+N_{k+1}+M_{k+1}\right)-Q_{K}\left(c_{k}+N_{k}+M_{k}\right)\right]\right. \\
\left.\times\left[Q_{K}\left(c_{k+m+1}+N_{k+m+1}+M_{k+m+1}\right)-Q_{K}\left(c_{k+m}+N_{k+m}+M_{k+m}\right)\right]\right\} \\
\quad \frac{1}{4} b_{k} b_{k+m} E_{n}\left\{Q_{K}\left(c_{k+1}+N_{k+1}+M_{k+1}\right) Q_{K}\left(c_{k+m+1}+N_{k+m+1}+M_{k+m+1}\right)\right\} \\
+\frac{1}{4} b_{k} b_{k+m} E_{n}\left\{Q_{K}\left(c_{k}+N_{k}+M_{k}\right) Q_{K}\left(c_{k+m}+N_{k+m}+M_{k+m}\right)\right\}
\end{aligned}
$$

W We again omit the conditioning on $\lambda$ in our notation, assuming it to be understood. 


$$
\begin{aligned}
& -\frac{1}{4} b_{k} b_{k+m} E_{n}\left\{Q_{K}\left(c_{k}+N_{k}+M_{k}\right) Q_{K}\left(c_{k+m+1}+N_{k+m+1}+M_{k+m+1}\right)\right\} \\
& -\frac{1}{4} b_{k} b_{k+m} E_{n}\left\{Q_{K}\left(c_{k+1}+N_{k+1}+M_{k+1}\right) Q_{K}\left(c_{k+m}+N_{k+m}+M_{k+m}\right)\right\}
\end{aligned}
$$

But,

$$
\begin{gathered}
E\left\{Q_{K}(c+x) Q_{K}(d+y)\right\}=\operatorname{erf}_{K}\left(\frac{c}{\sigma_{x} \sqrt{2}}\right) \operatorname{erf}_{K}\left(\frac{d}{\sigma_{y} \sqrt{2}}\right) \\
E\left\{Q_{K}^{2}(c+x)\right\}=1{ }_{K}^{(2)}\left(\frac{c}{\sigma_{x} \sqrt{2}}\right)
\end{gathered}
$$

Thus,

$\underline{F o r} \mathrm{~m}=0$

$$
\text { Term No. } 1=\frac{b_{k}^{2}}{4}\left[1_{K}^{(2)}\left(\frac{c_{k}}{\sigma_{\nu} \sqrt{2}}\right)+1_{K}^{(2)}\left(\frac{c_{k+1}}{\sigma_{\nu} \sqrt{2}}\right)-2 \operatorname{erf}{ }_{K}\left(\frac{c_{k}}{\sigma_{\nu} \sqrt{2}}\right) \operatorname{erf}{ }_{K}\left(\frac{c_{k+1}}{\sigma_{\nu} \sqrt{2}}\right)\right]
$$

For $m=1$

$$
\begin{aligned}
\text { Term No. } 1= & \frac{1}{4} b_{k} b_{k+1}\left\{\operatorname{erf}_{K}\left(\frac{c_{k+1}}{\sigma_{v} \sqrt{2}}\right) \operatorname{erf}\left(\frac{c_{k}+2}{\sigma_{v} \sqrt{2}}\right)\right\} \\
& +\frac{1}{4} b_{k} b_{k+1}\left\{\operatorname{erf}_{K}\left(\frac{c_{k}}{\sigma_{v} \sqrt{2}}\right) \operatorname{erf}\left(\frac{c_{k+1}}{\sigma_{v} \sqrt{2}}\right)\right\} \\
& -\frac{1}{4} b_{k} b_{k+1}\left\{\operatorname{erf}_{K}\left(\frac{c_{k}}{\sigma_{v} \sqrt{2}}\right) \operatorname{erf}\left(\frac{c_{k+2}}{\sigma_{v} \sqrt{2}}\right)\right\}-\frac{1}{4} b_{k} b_{k+1} l_{K}^{(2)}\left(\frac{c_{k+1}}{\sigma_{v} \sqrt{2}}\right)
\end{aligned}
$$

B -2

JPL Technical Memorandum 33-720 
TERM NO. 2

$$
\begin{aligned}
\frac{1}{4} b_{k} E_{n} & \left\{M_{k+m}^{\prime}\left[Q_{K}\left(c_{k+1}+N_{k+1}+M_{k+1}\right)-Q_{K}\left(c_{k}+N_{k}+M_{k}\right)\right]\right. \\
& \left.x\left[Q_{K}\left(c_{k+m+1}+N_{k+m+1}+M_{k+m+1}\right)-Q_{K}\left(c_{k+m}+N_{k+m}+M_{k+m}\right)\right]\right\}
\end{aligned}
$$

Defining

$$
\begin{aligned}
& E_{n}\left\{M_{k}^{\prime} Q_{K}\left(c_{k}+M_{k}+N_{k}\right)\right\}=\xi \frac{\sigma_{v}}{\sqrt{2 \pi}} \exp _{K}\left(\frac{-c_{k}^{2}}{2 \sigma_{v}^{2}}\right) \\
& E_{n}\left\{M_{k}^{\prime} Q_{K}^{2}\left(c_{k}+M_{k}+N_{k}\right)\right\}=\xi \frac{\sigma_{v}}{\sqrt{2 \pi}} O_{K}^{(2)}\left(\frac{-c_{k}^{2}}{2 \sigma_{v}^{2}}\right)
\end{aligned}
$$

where $\exp _{\mathrm{K}}\left(-\mathrm{C}^{2} / 2 \sigma_{\mathrm{y}}^{2}\right)$ and $0_{\mathrm{K}}^{(2)}\left(-\mathrm{C}^{2} / 2 \sigma_{\mathrm{y}}^{2}\right)$ are given by $(E-11)$ and $(E-18)$ respectively, then,

For $m=0$

$$
\operatorname{Term} \text { No. } 2=\frac{b_{k}}{4} \xi \frac{\sigma_{v}}{\sqrt{2 \pi}}\left[0 \frac{(2)}{K}\left(\frac{c_{k}^{2}}{2 \sigma_{v}^{2}}\right)-2 \operatorname{erf}_{K}\left(\frac{c_{k+1}}{\sigma_{v} \sqrt{2}}\right) \exp _{K}\left(\frac{-c_{k}^{2}}{2 \sigma_{v}^{2}}\right)\right]
$$

For $\mathrm{m}=1$

Term No. $2=\frac{1}{4} b_{k} \xi \frac{\sigma_{v}}{\sqrt{2 \pi}}\left[\operatorname{erf}_{K}\left(\frac{c_{k+2}}{\sigma_{v} \sqrt{2}}\right)+\operatorname{erf}_{K}\left(\frac{c_{k}}{\sigma_{v} \sqrt{2}}\right)\right] \exp _{K}\left(\frac{-c_{k+1}^{2}}{2 \sigma_{v}^{2-}}\right)$ 
TERM NO. 3

$$
\begin{aligned}
\frac{1}{4} b_{k} E_{n} & \left\{N_{k+m+1}^{\prime}\left[Q_{K}\left(c_{k+1}+N_{k+1}+M_{k+1}\right)-Q_{K}\left(c_{k}+N_{k}+M_{k}\right)\right]\right. \\
\times & {\left.\left[Q_{K}\left(c_{k+m+1}+N_{k+m+1}+M_{k+m+1}\right)-Q_{K}\left(c_{k+m}+N_{k+m}+M_{k+m}\right)\right]\right\} }
\end{aligned}
$$

By comparison with Term No. 2, we write:

For $m=0$

$$
\left.\operatorname{Term} \text { No. } 3=\frac{b_{k}}{4} \xi \frac{\sigma_{v}}{\sqrt{2 \pi}}\left[0 \frac{0_{K}^{(2)}}{c_{k+1}^{2}} \frac{c_{k}^{2}}{2 \sigma_{v}^{2}}\right)-2 \operatorname{erf}_{K}\left(\frac{c_{k}}{\sigma_{v} \sqrt{2}}\right) \exp _{K}\left(\frac{-c_{k+1}^{2}}{2 \sigma_{v}^{2}}\right)\right]
$$

For $m=1$

$$
\operatorname{Term} \text { No. } 3=\frac{1}{4} b_{k} \xi \frac{\sigma_{v}}{\sqrt{2 \pi}}\left[\operatorname{erf}\left(\frac{c_{k+1}}{\sigma_{v} \sqrt{2}}\right)-\operatorname{erf}_{K}\left(\frac{c_{k}}{\sigma_{v} \sqrt{2}}\right)\right] \exp P_{K}\left(\frac{-c_{k+2}^{2}}{2 \sigma_{v}^{2}}\right)
$$

TERM NO, 4

$$
\begin{aligned}
\frac{1}{4} b_{k+m} E_{n} & \left\{M_{k}^{\prime}\left[Q_{K}\left(c_{k+1}+N_{k+1}+M_{k+1}\right)-Q_{K}\left(c_{k}+N_{k}+M_{k}\right)\right]\right. \\
& \left.\times\left[Q_{K}\left(c_{k+m+1}+N_{k+m+1}+M_{k+m+1}\right)-Q_{K}\left(c_{k+m}+N_{k+m}+M_{k+m}\right)\right]\right\}
\end{aligned}
$$

For $m=0$

Same as Term No. 2 


\section{For $m=1$}

Term No. $4=\frac{1}{4} b_{k+1} \xi \frac{\sigma_{v}}{\sqrt{2 \pi}}\left[\operatorname{erf}_{K}\left(\frac{c_{k+1}}{\sigma_{\nu} \sqrt{2}}\right)-\operatorname{erf}_{K}\left(\frac{c_{k+2}}{\sigma_{v} \sqrt{2}}\right)\right] \exp _{K}\left(\frac{-c_{k}^{2}}{2 \sigma_{v}^{2}}\right)$

TERM NO. 5

$$
\begin{aligned}
\frac{1}{4} b_{k+m} E_{n} & \left\{N_{k+1}^{\prime}\left[Q_{K}\left(c_{k+1}+N_{k+1}+M_{k+1}\right)-Q_{K}\left(c_{k}+N_{k}+M_{k}\right)\right]\right. \\
x & {\left.\left[Q_{K}\left(c_{k+m+1}+N_{k+m+1}+M_{k+m+1}\right)-Q_{K}\left(c_{k+m}+N_{k+m}+M_{k+m}\right)\right]\right\} }
\end{aligned}
$$

For $m=0$

$$
\text { Same as Term No. } 3
$$

For $m=1$

$$
\frac{1}{4} b_{k+1} \xi \frac{\sigma_{v}}{\sqrt{2 \pi}}\left[\operatorname{erf}_{K}\left(\frac{c_{k+2}}{\sigma_{v} \sqrt{2}}\right)+\operatorname{erf}_{K}\left(\frac{c_{k}}{\sigma_{v} \sqrt{2}}\right)\right] \exp _{K}\left(\frac{-c_{k+1}^{2}}{2 \sigma_{\nu}^{2}}\right)
$$

TERM NO. 6

$$
\begin{aligned}
\frac{1}{4} E_{n} & \left\{M_{k}^{\prime} M_{k+m}^{\prime}\left[Q_{K}\left(c_{k+1}+N_{k+1}+M_{k+1}\right)-Q_{K}\left(c_{k}+N_{k}+M_{k}\right)\right]\right. \\
x & {\left.\left[Q_{K}\left(c_{k+m+1}+N_{k+m+1}+M_{k+m+1}\right)-Q_{K}\left(c_{k+m}+N_{k+m}+M_{k+m}\right)\right]\right\} }
\end{aligned}
$$

\section{Defining}

$$
E\left\{M_{k}^{2} Q_{K}^{2}\left(c_{k}+M_{k \cdot}+N_{k}\right)\right\}=\sigma_{K}^{\prime 2}\left(\frac{c_{k}}{\sqrt{2} \sigma_{v}}\right)
$$


where from $(E-27)$ and $(E-28)$

$$
\sigma_{K}^{\prime 2}\left(\frac{c_{k}}{\sqrt{2} \sigma_{\nu}}\right)=\sigma^{\prime 2}\left[{ }_{K}^{(2)}\left(\frac{c_{k}}{\sqrt{2} \sigma_{\nu}}\right)-\frac{\xi}{\sqrt{\pi}}\left(\frac{c_{k}}{\sqrt{2} \sigma_{\nu}}\right)_{K} 0_{K}^{(2)}\left(\frac{-c_{k}^{2}}{2 \sigma_{\nu}^{2}}\right)\right]
$$

with $\sigma^{2}$ given in (14) of the main text, then

For $\mathrm{m}=0$

Term No. $6=\frac{1}{4} E_{n}\left\{M_{K}^{2}\left[Q_{K}^{2}\left(c_{k+1}+N_{k+1}+M_{k+1}\right)+Q_{K}^{2}\left(c_{k}+N_{k}+M_{k}\right)\right.\right.$

$$
\left.\left.-2 Q_{K}\left(c_{k}+N_{k}+M_{k}\right) Q_{K}\left(c_{k+1}+N_{k+1}+M_{k+1}\right)\right]\right\}
$$

Using $(B-20)$, we get

$$
\text { Term No. } \begin{aligned}
\sigma= & \frac{\sigma^{2}}{4}\left[\frac{\sigma_{K}^{\prime 2}\left(\frac{c_{k}}{\sqrt{2} \sigma_{\nu}}\right)}{\sigma^{t^{2}}}+1_{K}^{(2)}\left(\frac{c_{k+1}}{\sqrt{2} \sigma_{\nu}}\right)-2 \operatorname{erf} K\left(\frac{c_{k+1}}{\sigma_{\nu} \sqrt{2}}\right)\right. \\
& \left.x\left\{\operatorname{erf}_{K}\left(\frac{c_{k}}{\sigma_{\nu} \sqrt{2}}\right)-\frac{1}{\sqrt{\pi}} \xi\left(\frac{c_{k}}{\sigma_{\nu} \sqrt{2}}\right)_{K} \exp _{K}\left(\frac{-c_{k}^{2}}{2 \sigma_{\nu}^{2}}\right)\right\}\right]
\end{aligned}
$$

For $m=1$

$$
\text { Term No. } 6=\frac{1}{4}\left(\xi \frac{\sigma_{\nu}}{\sqrt{2 \pi}}\right)^{2} \exp _{K}\left(\frac{-c_{k}^{2}}{2 \sigma_{\nu}^{2}}\right) \exp _{K}\left(\frac{-c_{k+1}^{2}}{2 \sigma_{\nu}^{2}}\right)
$$


TERM NO. 7

$$
\begin{aligned}
\frac{1}{4} E_{n} & \left\{N_{k+1}^{\prime} N_{k+m+1}^{\prime}\left[Q_{K}\left(c_{k+1}+N_{k+1}+M_{k+1}\right)-Q_{K}\left(c_{k}+N_{k}+M_{k}\right)\right]\right. \\
x & {\left.\left[Q_{K}\left(c_{k+m+1}+N_{k+m+1}+M_{k+m+1}\right)-Q_{K}\left(c_{k+m}+N_{k+m}+M_{k+m}\right)\right]\right\} }
\end{aligned}
$$

By comparison with Term No. 6,

For $m=0$

$$
\begin{aligned}
& \text { Term No. } 7=\frac{\sigma^{\prime}}{4}\left[\frac{{ }_{\mathrm{K}}^{\prime 2}\left(\frac{{ }^{c} k+1}{\sqrt{2} \sigma_{v}}\right)}{\sigma^{\prime 2}}+1_{K}^{(2)}\left(\frac{c_{k}}{\sqrt{2} \sigma_{v}}\right)-2 \operatorname{erf}\left(\frac{c_{k}}{\sigma_{v} \sqrt{2}}\right)\right. \\
& x\left\{\operatorname{erf} K\left(\frac{c_{k+1}}{\sigma_{\nu} \sqrt{2}}\right)-\frac{1}{\sqrt{\pi}} \xi\left(\frac{c_{k+1}}{\sigma_{\nu} \sqrt{2}}\right)_{K} \exp _{K}\left(\frac{-c_{k+1}^{2}}{2 \sigma_{\nu}^{2}}\right)\right\}(B-26)
\end{aligned}
$$

For $m=1$

$$
\text { Term No. } 7=\frac{1}{4}\left(\xi \frac{\sigma_{v}}{\sqrt{2 \pi}}\right)^{2} \exp _{\mathrm{K}}\left(\frac{-\mathrm{c}_{\mathrm{k}+1}^{2}}{2 \sigma_{i}^{2}}\right) \exp _{\mathrm{K}}\left(\frac{-\mathrm{c}_{\mathrm{k}+2}^{2}}{2 \sigma_{v}^{2}}\right)
$$

TERM NO. 8

$$
\begin{aligned}
\frac{1}{4} E_{n} & \left\{N_{k+1}^{\prime} M_{k+m}^{\prime}\left[Q_{K}\left(c_{k+1}+N_{k+1}+M_{k+1}\right)-Q_{K}\left(c_{k}+N_{k}+M_{k}\right)\right]\right. \\
& \left.\times\left[Q_{K} \cdot\left(c_{k+m+1}+N_{k+m+1}+M_{k+m+1}\right)-Q_{K}\left(c_{k+m}+N_{k+m}+M_{k+m}\right)\right]\right\}
\end{aligned}
$$


For $m=0$

$$
\text { Term No. } 8=-\frac{1}{2}\left(\xi \frac{\sigma_{\nu}}{\sqrt{2 \pi}}\right)^{2} \exp _{K}\left(\frac{-c_{k+1}^{2}}{2 \sigma_{v}^{2}}\right) \exp _{K}\left(\frac{-c_{k}^{2}}{2 \sigma_{v}^{2}}\right)
$$

For $m=1$

Term No. $8=-\frac{\sigma^{\prime}}{4 \sqrt{\pi}}\left\{\left[\operatorname{erf}_{K}\left(\frac{c_{k}}{\sqrt{2} \sigma_{\nu}}\right)+\operatorname{erf}_{K}\left(\frac{c_{k+2}}{\sqrt{2} \sigma_{\nu}}\right)\right]\left(\frac{c_{k+1}}{\sqrt{2} \sigma_{\nu}}\right)_{K} \exp _{K}\left(-\frac{c_{k+1}^{2}}{2 \sigma_{\nu}^{2}}\right)\right.$

$$
\left.-\left(\frac{c_{k+1}}{\sqrt{2} \sigma_{\nu}}\right)_{K} 0_{K}^{(2)}\left(-\frac{c_{k+1}^{2}}{2 \sigma_{\nu}^{2}}\right)\right\}
$$

TERM NO. 9

$$
\begin{aligned}
\frac{1}{4} E_{n} & \left\{M_{k}^{\prime} N_{k+m+1}^{\prime}\left[Q_{K}\left(c_{k+1}+N_{k+1}+M_{k+1}\right)-Q_{K}\left(c_{k}+N_{k}+M_{k}\right)\right]\right. \\
& \left.x\left[Q_{K}\left(c_{k+m+1}+N_{k+m+1}+M_{k+m+1}\right)-Q_{K}\left(c_{k+m}+N_{k+m}+M_{k+m}\right)\right]\right\}
\end{aligned}
$$

For $m=0$

$$
\text { Same as Term No. } 8
$$

For $m=1$

$$
\text { Term No. } 9=-\frac{1}{4}\left(\xi \frac{\sigma_{v}}{\sqrt{2 \pi}}\right)^{2} \exp _{K}\left(\frac{-c_{k}^{2}}{2 \sigma_{v}^{2}}\right) \exp _{K}\left(\frac{-c_{k+2}^{2}}{2 \sigma_{v}^{2}}\right)
$$


TERM NO. 10

$$
\begin{aligned}
\frac{\xi^{2}}{16} E_{n}\{ & {\left[Q_{K}^{2}\left(c_{k}+M_{k}+N_{k}\right)-Q_{K}^{2}\left(c_{k+1}+M_{k+1}+N_{k+1}\right)\right] } \\
& \left.x\left[Q_{K}^{2}\left(c_{k+m}+M_{k+m}+N_{k+m}\right)-Q_{K}^{2}\left(c_{k+m+1}+M_{k+m+1}+N_{k+m+1}\right)\right]\right\}
\end{aligned}
$$

For $\mathrm{m}=0$

$\operatorname{Term~No.~} 10=\frac{\xi^{2}}{16}\left[1_{\mathrm{K}}^{(4)}\left(\frac{c_{k}}{\sqrt{2} \sigma_{v}}\right)+1_{\mathrm{K}}^{(4)}\left(\frac{c_{k+1}}{\sqrt{2} \sigma_{v}}\right)-21_{\mathrm{K}}^{(2)}\left(\frac{c_{k}}{\sqrt{2} \sigma_{v}}\right) 1_{K}^{(2)}\left(\frac{c_{k+1}}{\sqrt{2} \sigma_{v}}\right)\right]$

For $\mathrm{m}=1$

$$
\begin{aligned}
\text { Term No. } 10= & \frac{\xi^{2}}{16}\left[1_{\mathrm{K}}^{(2)}\left(\frac{\mathrm{c}_{\mathrm{k}}}{\sqrt{2} \sigma_{\nu}}\right) 1_{\mathrm{K}}^{(2)}\left(\frac{\mathrm{c}_{\mathrm{k}+1}}{\sqrt{2} \sigma_{\nu}}\right)-1_{\mathrm{K}}^{(2)}\left(\frac{\mathrm{c}_{\mathrm{k}}}{\sqrt{2} \sigma_{v}}\right) 1_{\mathrm{K}}^{(2)}\left(\frac{\mathrm{c}_{\mathrm{k}+2}}{\sqrt{2} \sigma_{v}}\right)\right. \\
& \left.+1_{\mathrm{K}}^{(2)}\left(\frac{\mathrm{c}_{\mathrm{k}+1}}{\sqrt{2} \sigma_{v}}\right) 1_{\mathrm{K}}^{(2)}\left(\frac{\mathrm{c}_{\mathrm{k}+2}}{\sqrt{2} \sigma_{v}}\right)-1_{\mathrm{K}}^{(4)}\left(\frac{\mathrm{c}_{\mathrm{k}+1}}{\sqrt{2} \sigma_{v}}\right)\right]
\end{aligned}
$$

TERM NO. 11

$$
\begin{aligned}
-\frac{\xi b_{k+m}}{8} E_{n}\left\{\left[Q_{K}^{2}\left(c_{k}+M_{k}+N_{k}\right)-Q_{K}^{2}\left(c_{k+1}+M_{k+1}+N_{k+1}\right)\right]\right. \\
\left.\times\left[Q_{K}\left(c_{k+m}+M_{k+m}+N_{k+m}\right)-Q_{K}\left(c_{k+m+1}+M_{k+m+1}+N_{k+m+1}\right)\right]\right\}
\end{aligned}
$$


For $\mathrm{m}=0$

$$
\begin{aligned}
\operatorname{Term} \text { No. } 11= & -\frac{\xi_{\mathrm{k}}}{8}\left[1_{\mathrm{K}}^{(3)}\left(\frac{\mathrm{c}_{\mathrm{k}}}{\sqrt{2} \sigma_{\nu}}\right)+1_{\mathrm{K}}^{(3)}\left(\frac{\mathrm{c}_{\mathrm{k}+1}}{\sqrt{2} \sigma_{\nu}}\right)-1_{\mathrm{K}}^{(2)}\left(\frac{\mathrm{c}_{\mathrm{k}}}{\sqrt{2} \sigma_{\nu}}\right) \operatorname{erf}_{\mathrm{K}}\left(\frac{\mathrm{c}_{\mathrm{k}+1}}{\sqrt{2} \sigma_{\nu}}\right)\right. \\
& \left.-1_{\mathrm{K}}^{(2)}\left(\frac{\mathrm{c}_{\mathrm{k}+1}}{\sqrt{2} \sigma_{\nu}}\right) \operatorname{erf}_{\mathrm{K}}\left(\frac{\mathrm{c}_{\mathrm{k}}}{\sqrt{2} \sigma_{\nu}}\right)\right]
\end{aligned}
$$

For $\mathrm{m}=1$

$$
\begin{aligned}
\text { Term No. II }= & -\frac{\xi b_{k+1}}{8}\left\{1_{K}^{(2)}\left(\frac{c_{k}}{\sqrt{2} \sigma_{v}}\right)\left[\operatorname{erf}\left(\frac{c_{k+1}}{\sqrt{2} \sigma_{v}}\right)-\operatorname{erf}\left(\frac{c_{k+2}}{\sqrt{2} \sigma_{v}}\right)\right]\right. \\
& \left.-1_{K}^{(3)}\left(\frac{c_{k+1}}{\sqrt{2} \sigma_{v}}\right)+1_{K}^{(2)}\left(\frac{c_{k+1}}{\sqrt{2} \sigma_{v}}\right) \operatorname{erf}\left(\frac{c_{k+2}}{\sqrt{2} \sigma_{v}}\right)\right\}
\end{aligned}
$$

TERM NO. 12

$$
\begin{aligned}
-\frac{\xi}{8} E_{n} & \left\{M_{k+m}^{\prime}\left[Q_{K}^{2}\left(c_{k}+M_{k}+N_{k}\right)-Q_{K}^{2}\left(c_{k+1}+M_{k+1}+N_{k+1}\right)\right]\right. \\
& \left.x\left[Q_{K}\left(c_{k+m}+M_{k+m}+N_{k+m}\right)-Q_{K}\left(c_{k+m+1}+M_{k+m+1}+N_{k+m+1}\right)\right]\right\}
\end{aligned}
$$

For $\mathrm{m}=0$

$$
\begin{aligned}
\text { Term No. } 12= & -\frac{\xi^{2} \sigma_{v}}{8 \sqrt{2 \pi}}\left[\exp _{\mathrm{K}}^{(3)}\left(-\frac{\mathrm{c}_{\mathrm{k}}^{2}}{2 \sigma_{v}^{2}}\right)-\operatorname{erf}_{\mathrm{K}}\left(\frac{\mathrm{c}_{\mathrm{k}+1}}{\sqrt{2} \sigma_{v}}\right) 0_{\mathrm{K}}^{(2)}\left(-\frac{\mathrm{c}_{\mathrm{k}}^{2}}{2 \sigma_{v}^{2}}\right)\right. \\
& \left.-1_{\mathrm{K}}^{(2)}\left(\frac{\mathrm{c}_{\mathrm{k}+1}}{\sqrt{2} \sigma_{v}}\right) \exp _{\mathrm{K}}\left(-\frac{\mathrm{c}_{\mathrm{k}}^{2}}{2 \sigma_{v}^{2}}\right)\right]
\end{aligned}
$$


For $m=1$

$$
\text { Term No. } \begin{aligned}
12= & \frac{\xi^{2} \sigma_{v}}{8 \sqrt{2 \pi}}\left[\exp _{\mathrm{K}}^{(3)}\left(-\frac{c_{\mathrm{k}+1}^{2}}{2 \sigma_{v}^{2}}\right)-\operatorname{erf}_{\mathrm{K}}\left(\frac{\mathrm{c}_{\mathrm{k}+2}}{\sqrt{2} \sigma_{v}}\right) 0_{\mathrm{K}}^{(2)}\left(-\frac{\mathrm{c}_{\mathrm{k}+1}^{2}}{2 \sigma_{v}^{2}}\right)\right. \\
& \left.-1_{\mathrm{K}}^{(2)}\left(\frac{c_{k}}{\sqrt{2} \sigma_{v}}\right) \exp _{\mathrm{K}}\left(-\frac{\mathrm{c}_{\mathrm{k}+1}^{2}}{2 \sigma_{v}^{2}}\right)\right]
\end{aligned}
$$

TERM NO. 13

$$
\begin{aligned}
-\frac{\xi}{8} E_{n}\left\{N_{k+m+1}^{1}\left[Q_{K}^{2}\left(c_{k}+M_{k}+N_{k}\right)-Q_{K}^{2}\left(c_{k+1}+M_{k+1}+N_{k+1}\right)\right]\right. \\
\left.\times\left[Q_{K}\left(c_{k+m}+M_{k+m}+N_{k+m}\right)-Q_{K}\left(c_{k+m+1}+M_{k+m+1}+N_{k+m+1}\right)\right]\right\}
\end{aligned}
$$

For $\mathrm{m}=0$

$$
\begin{aligned}
\text { Term No. } 13= & -\frac{\xi^{2} \sigma_{\nu}}{8 \sqrt{2 \pi}}\left[\exp _{\mathrm{K}}^{(3)}\left(-\frac{\mathrm{c}_{\mathrm{k}+1}^{2}}{2 \sigma_{\nu}^{2}}\right)-\operatorname{erf}_{\mathrm{K}}\left(\frac{\mathrm{c}_{\mathrm{k}}}{\sqrt{2 \sigma_{\nu}}}\right) 0_{\mathrm{K}}^{(2)}\left(-\frac{\mathrm{c}_{\mathrm{k}+1}^{2}}{2 \sigma_{\nu}^{2}}\right)\right. \\
& \left.-\mathbf{1}_{\mathrm{K}}^{(2)}\left(\frac{\mathrm{c}_{\mathrm{k}}}{\sqrt{2} \sigma_{v}}\right) \exp _{\mathrm{K}}\left(-\frac{\mathrm{c}_{\mathrm{k}+1}^{2}}{2 \sigma_{\nu}^{2}}\right)\right]
\end{aligned}
$$

For $\mathrm{m}=1$

$$
\text { Term No. } 13=-\frac{\xi^{2} \sigma_{\nu}}{8 \sqrt{2 \pi}} \exp _{\mathrm{K}}\left(-\frac{\mathrm{c}_{\mathrm{k}+2}^{2}}{2 \sigma_{\nu}^{2}}\right)\left[1_{\mathrm{K}}^{(2)}\left(\frac{\mathrm{c}_{\mathrm{k}}}{\sqrt{2} \sigma_{\nu}}\right)-\dot{1}_{\mathrm{K}}^{(2)}\left(\frac{\mathrm{c}_{\mathrm{k}+1}}{\sqrt{2} \sigma_{\nu}}\right)\right]
$$


TERM NO. 14

$$
\begin{gathered}
-\frac{\xi b_{k}}{8} E_{n}\left\{\left[Q_{K}^{2}\left(c_{k+m}+M_{k+m}+N_{k+m}\right)-Q_{K}^{2}\left(c_{k+m+1}+M_{k+m+1}+N_{k+m+1}\right)\right]\right. \\
\left.x\left[Q_{K}\left(c_{k}+M_{k}+N_{k}\right)-Q_{K}\left(c_{k+1}+M_{k+1}+N_{k+1}\right)\right]\right\}
\end{gathered}
$$

For $\mathrm{m}=0$

For $\mathrm{m}=1$

Term No. $14=-\frac{\xi b_{k}}{8}\left\{\left[1_{\mathrm{K}}^{(2)}\left(\frac{c_{k+1}}{\sqrt{2} \sigma_{\nu}}\right)-1_{\mathrm{K}}^{(2)}\left(\frac{\mathrm{c}_{\mathrm{k}+2}}{\sqrt{2} \sigma_{\nu}}\right)\right] \operatorname{erf}{ }_{\mathrm{K}}\left(\frac{\mathrm{c}_{\mathrm{k}}}{\sqrt{2 \sigma_{\nu}}}\right)-1_{\mathrm{K}}^{(3)}\left(\frac{\mathrm{c}_{\mathrm{k}+1}}{\sqrt{2} \sigma_{\nu}}\right)\right.$

$$
\left.+1_{\mathrm{K}}^{(2)}\left(\frac{\mathrm{c}_{\mathrm{k}+2}}{\sqrt{2} \sigma_{\nu}}\right) \operatorname{erf} \mathrm{K}_{\mathrm{K}}\left(\frac{\mathrm{c}_{\mathrm{k}+1}}{\sqrt{2} \sigma_{\nu}}\right)\right\}
$$

TERM NO. 15

$$
\begin{aligned}
-\frac{\xi}{8} E_{n} & \left\{M_{k}^{1}\left[Q_{K}^{2}\left(c_{k+m}+M_{k+m}+N_{k+m}\right)-Q_{K}^{2}\left(c_{k+m+1}+M_{k+m+1}+N_{k+m+1}\right)\right]\right. \\
& \left.\times\left[Q_{K}\left(c_{k}+M_{k}+N_{k}\right)-Q_{K}\left(c_{k+1}+M_{k+1}+N_{k+1}\right)\right]\right\}
\end{aligned}
$$

For $\mathrm{m}=0$

For $\mathrm{m}=1$

$$
\text { Term No. } 15=-\frac{\xi^{2} \sigma_{v}}{8 \sqrt{2 \pi}} \exp _{\mathrm{K}}\left(-\frac{\mathrm{c}_{\mathrm{k}}^{2}}{2 \sigma_{\nu}^{2}}\right)\left[1_{\mathrm{K}}^{(2)}\left(\frac{\mathrm{c}_{\mathrm{k}+1}}{\sqrt{2} \sigma_{\nu}}\right)-1_{\mathrm{K}}^{(2)}\left(\frac{\mathrm{c}_{\mathrm{k}+2}}{\sqrt{2} \sigma_{v}}\right)\right]
$$


TERM NO. 16

$$
\begin{aligned}
-\frac{\xi}{8} E_{n} & \left\{N_{k+1}^{\prime}\left[Q_{K}^{2}\left(c_{k+m}+M_{k+m}+N_{k+m}\right)-Q_{K}^{2}\left(c_{k+m+1}+M_{k+m+1}+N_{k+m+1}\right)\right]\right. \\
& \left.\times\left[Q_{K}\left(c_{k}+M_{k}+N_{k}\right)-Q_{K}\left(c_{k+1}+M_{k+1}+N_{k+1}\right)\right]\right\}
\end{aligned}
$$

For $\mathrm{m}=0$

$$
\text { Same as Term No. } 13
$$

For $\mathrm{m}=1$

$$
\begin{aligned}
\text { Term No. } 16= & \frac{\xi_{\sigma_{v}}^{2}}{8 \sqrt{2} \pi}\left[\exp _{\mathrm{K}}^{(3)}\left(-\frac{\mathrm{c}_{\mathrm{k}+1}^{2}}{2 \sigma_{v}^{2}}\right)-\operatorname{erf}\left(\frac{\mathrm{c}_{\mathrm{k}}}{\sqrt{2} \sigma_{\nu}}\right) 0_{\mathrm{K}}^{(2)}\left(\frac{\mathrm{c}_{\mathrm{k}+1}}{\sqrt{2} \sigma_{\nu}}\right)\right. \\
& \left.-1 \frac{(2)}{\mathrm{K}}\left(\frac{\mathrm{c}_{\mathrm{k}+2}}{\sqrt{2} \sigma_{\nu}}\right) \exp _{K}\left(-\frac{\mathrm{c}_{\mathrm{k}+1}^{2}}{2 \sigma^{2}}\right)\right]
\end{aligned}
$$

We must now average each of the terms $(B-2)$ through $(B-54)$, over the signal distribution. The procedure will be to collect all terms corresponding to a given value of $\mathrm{m}$, and then average over all possible sequences where $c_{k}$, $c_{k+1}$, and $b_{k}$ are defined in $(A-18),(A-19)$ and in addition

$$
\begin{aligned}
& \frac{c_{k+m}}{\delta}=d_{k+m-2} \lambda+d_{k+m-1}(1-\lambda) \\
& \frac{c_{k+m+1}}{\delta}=d_{k+m-1} \lambda+d_{k+m}(1-\lambda) \quad \text { for } \lambda \leq \frac{\xi}{2} \\
& \frac{b_{k+m}}{\delta}=d_{k+m-1}\left[\frac{\xi}{2}+\lambda\right]+d_{k+m}\left[\frac{\xi}{2}-\lambda\right]
\end{aligned}
$$




$$
\begin{aligned}
& \frac{c_{k+m}}{\delta}=d_{k+m-2} \lambda+d_{k+m-1}(1-\lambda) \\
& \frac{c_{k+m+1}}{\delta}=d_{k+m-1} \lambda+d_{k+m}(1-\lambda) \quad \text { for } \frac{\xi}{2} \leq \lambda \leq \frac{1}{2} \\
& \frac{b_{k+m}}{\delta}=d_{k+m-1} \xi
\end{aligned}
$$

Summing first over the first nine terms, i.e., (B-2) through (B-33), and evaluating the results at $\lambda=0$ gives ${ }^{*}$

For $m=0$

$$
\begin{aligned}
& \frac{E_{n, s}\left\{e_{k}^{2}\right\}}{\sigma_{v}^{4} \xi R_{d}}=\frac{3(K-1)^{2}}{K^{2}-1}+(K-1)^{2} \xi R_{d}^{\prime}-\frac{12 \xi}{K^{2}\left(K^{2}-1\right)} \\
& \left.x\left[\sqrt{R_{d}^{\prime}} \sum_{m} m \sum_{\ell} \operatorname{erf}\left[(m-\ell) \sqrt{R_{d}^{\prime}}\right] \frac{\sum_{m} \sum_{\ell} \exp \left[-(m-\ell)^{2} R_{d}^{\prime}\right.}{\sqrt{\pi}}\right]\right]^{2} \\
& +\frac{3}{K^{2}-1} \sum_{m}\left[\frac{2}{K} \xi R_{d}^{\prime}\left(m^{2}+\frac{K^{2}-1}{3}+\frac{4}{K}\right)\right] \sum_{\ell} \ell \operatorname{erf}\left[(m-\ell) \sqrt{R_{d}^{1}}\right] \\
& +\frac{12}{K\left(K^{2}-1\right)} \xi \sqrt{\frac{R_{d}^{\prime}}{\pi}} \sum_{m} m \sum_{\ell} \ell \exp \left[-(m-\ell)^{2} R_{d}^{\prime}\right] \\
& -\frac{3}{K\left(K^{2}-1\right)} \xi \sqrt{\frac{R_{d}^{\prime}}{\pi}} \sum_{m} \sum_{\ell} \ell(m-\ell) \exp \left[-(m-\ell)^{2} R_{d}^{\prime}\right]
\end{aligned}
$$

The sum over $m$ is for values $m=1,3,5, \ldots,(K-1)$ while the sum over $\ell$ is for values $\ell=0, \pm 2, \pm 4, \cdots, \pm(\mathrm{K}-2)$. 
For $m=1$

$$
\begin{aligned}
& \frac{E_{n, s}\left\{e_{k} e_{k+1}\right\}}{\sigma_{v}^{4} \xi R_{d}}=\frac{3 \xi R_{d}^{\prime}}{K^{2}\left(K^{2}-1\right)}\left\{\mid\left[\sum_{m} m \sum_{\ell} \operatorname{erf}\left[(m-\ell) \sqrt{R_{d}^{\prime}}\right]\right]^{2}-\frac{K^{2}(K-1)^{2}\left(K^{2}-1\right)}{12}\right\} \\
& +\frac{6}{K^{2}\left(K^{2}-1\right)} \xi \sqrt{\frac{R_{d}^{\prime}}{\pi}} \sum_{m} m \sum_{\ell} \operatorname{erf}\left[(m-\ell) \sqrt{R_{d}^{\prime}}\right] \sum_{m^{\prime}} m^{\prime} \sum_{\ell^{\prime}} \exp \left[-\left(m^{\prime}-\ell^{\prime}\right)^{2} R_{d}^{\prime}\right] \\
& +\frac{3 \xi}{K^{2}\left(K^{2}-1\right) \pi}\left\{\sum_{m} \sum_{\ell} \exp \left[-(m-\ell)^{2} R_{d}^{1}\right]\right\}^{2} \\
& -\frac{3 \xi R_{d}^{\prime}}{K\left(K^{2}-1\right)} \sum_{m} m^{2} \sum_{\ell} \ell \operatorname{erf}\left[(m-\ell) \sqrt{R_{d}^{\prime}}\right] \\
& -\frac{6 \xi}{\mathrm{K}\left(\mathrm{K}^{2}-1\right)} \sqrt{\frac{\mathrm{R}_{\mathrm{d}}^{1}}{\pi}} \sum_{\mathrm{m}} \mathrm{m} \sum_{\ell} \ell \exp \left[-(\mathrm{m}-\ell)^{2} \mathrm{R}_{\mathrm{d}}^{1}\right] \\
& +\frac{3 \xi}{\mathrm{K}\left(\mathrm{K}^{2}-1\right)} \sqrt{\frac{\mathrm{R}_{\mathrm{d}}^{\prime}}{\pi}} \sum_{\mathrm{m}} \sum_{\ell} \ell(\mathrm{m}-\ell) \exp \left[-(\mathrm{m}-\ell)^{2} \mathrm{R}_{\mathrm{d}}^{\mathrm{r}}\right]
\end{aligned}
$$

Thus, for the first nine terms

$$
\begin{aligned}
& \frac{E_{n, s}\left\{e_{k}^{2}\right\}+2 E_{n, s}\left\{c_{k} c_{k+1}\right\}}{{ }^{4}{ }_{v} \xi R_{d}}=\frac{3(K-1)^{2}}{K^{2}-1}+\frac{(K-1)^{2}}{2} \xi R_{d}^{\prime} \\
& -\frac{6}{K^{2}\left(K^{2}-1\right)} \xi\left[\sqrt{R_{d}^{i}} \sum_{m} m \sum_{\ell} \operatorname{erf}\left[(m-\ell) \sqrt{R_{d}^{\prime}}\right]+\frac{\sum_{m} \sum_{\ell} \exp \left[-(m-\ell)^{2} R_{d}^{\prime}\right]}{\sqrt{\pi}}\right]^{2} \\
& +\frac{6}{K\left(K^{2}-1\right)}\left[2+\xi R_{d}^{\prime}\left(\frac{K^{2}-1}{3}\right)\right] \sum_{m} \sum_{\ell} \ell \operatorname{erf}\left[(m-\ell) \sqrt{R_{d}^{\prime}}\right]
\end{aligned}
$$


Summing up the contributions of terms ten through sixteen, i.e., (B-34) through $(B-45)$ and evaluating at $\lambda=0$ we find that

$$
\frac{E_{n, s}\left\{e_{k}^{2}\right\}+2 E_{n, s}\left\{e_{k} e_{k+1}\right\}}{\sigma_{v}^{4} \xi R_{d}}=0
$$

Combining (B-59) and (B-60) and recalling from (12) and (14) of the main text that $\sigma_{v}^{2}=\mathrm{N}_{\mathrm{o}} / 2 \mathrm{~T}$, then

$$
\begin{aligned}
& h(0) \triangleq \frac{S(0,0)}{\left(\frac{N_{0}}{2 T}\right)^{2} \xi R_{d}}=\frac{R(0,0)+2 R(1,0)}{\left(\frac{N o}{2 T}\right)^{2} \xi R_{d}}=\frac{3(K-1)^{2}}{K^{2}-1}+\frac{(K-1)^{2}}{2} \xi R_{d}^{\prime} \\
& \left.-\frac{6}{K^{2}\left(K^{2}-1\right)} \xi\left[\sqrt{R_{d}^{l}} \sum_{m} m \sum_{\ell} \operatorname{erf}\left|(m-\ell) \sqrt{R_{d}^{\prime}}\right|+\frac{\sum_{m} \sum_{\ell} \exp \left[-(m-\ell)^{2} R_{d}^{l}\right]}{\sqrt{\pi}}\right]\right]^{2} \\
& +\frac{6}{K\left(K^{2}-1\right)}\left[2+\xi R_{d}^{\prime}\left(\frac{K^{2}-1}{3}\right)\right] \sum_{m} \sum_{\ell} \ell \operatorname{erf}\left[(m-\ell) \sqrt{R_{d}^{1}}\right]
\end{aligned}
$$

where from (19) of the main text

$$
\begin{aligned}
& R(0,0) \triangleq E_{n, s}\left\{\left.e_{k}^{2}\right|_{\lambda=0}\right\} \\
& R(1,0) \triangleq E_{n, s}\left\{\left.e_{k} e_{k+1}\right|_{\lambda=0}\right\}
\end{aligned}
$$




\section{APPENDIX C}

SLOPE OF THE LOOP S-CURVE AT THE ORIGIN

The purpose of this appendix is to evaluate

$$
\left.K_{g} \triangleq \frac{\operatorname{dg}_{n}(\lambda)}{d \lambda}\right|_{\lambda=0}
$$

where $g_{n}(\lambda) \triangleq g(\lambda) / S$ with $g(\lambda)$ given by (11) of the main text. Differentiating (11) with respect to $\lambda$ and evaluating the result at $\lambda=0$ gives

$$
\begin{aligned}
K_{g}= & \frac{3}{2\left(K^{2}-1\right) K^{2}} \sum_{m, j, l} \epsilon_{\ell}\left\{( - j \frac { \xi } { 2 } ) 2 \sqrt { \frac { R _ { d } ^ { \prime } } { \pi } } \left[(j-m) \exp \left[-R_{d}^{\prime}(m+\ell)^{2}\right]\right.\right. \\
& -(-j+m) \exp \left[-R_{d}^{\prime}(-m+\ell)^{2}\right]+(j+m) \exp \left[-R_{d}^{\prime}(-m+\ell)^{2}\right] \\
& -(-j-m) \exp \left[-R_{d}^{\prime}(m+\ell)^{2}\right]+(2 m-j)\left[\operatorname { e r f } \left[\sqrt{\left.R_{d}^{\prime}(m+l)\right]}\right.\right. \\
& \left.-\operatorname{erf}\left[\sqrt{R_{d}^{\prime}(-m+l)}\right]\right]-(2 m+j)\left[\operatorname{erf}\left[\sqrt{R_{d}^{\prime}(-m+l)}\right]\right. \\
& -\operatorname{erf}\left[\sqrt{R_{d}^{\prime}(m+l)}\right]
\end{aligned}
$$


or

$$
\begin{aligned}
K_{g}= & \frac{3}{2\left(K^{2}-1\right) K^{2}} \sum_{m, j, \ell} \epsilon_{\ell}\left\{-j \xi \sqrt{\frac{R_{d}^{\prime}}{\pi}}\left[2 \exp \left[-R_{d}^{\prime}(m+\ell)^{2}\right]\right.\right. \\
& +2 \exp \left[-R_{d}^{\prime}(-m+\ell)^{2}\right]+4 m\left[\operatorname{erf}\left[\sqrt{R_{d}^{\prime}}(m+\ell)\right]\right. \\
& \left.-\operatorname{erf}\left[\sqrt{R_{d}^{\prime}}(-m+\ell)\right]\right]
\end{aligned}
$$

After considerable algebraic manipulation, we arrive at the simplified result

$$
\begin{aligned}
K_{g}= & \frac{3 K}{2\left(K^{2}-1\right)}\left\{_ { n = - K + 3 , - K + 5 , \cdots , - 1 } \sum _ { n = 1 , 3 , \cdots , K - 1 } \operatorname { e r f } \left(n \sqrt{\left.R_{d}^{\prime}\right)}\right.\right. \\
& +\sum_{n=K+1, K+3, \cdots, 2 K-3}\left[1-\frac{(n-K+1)^{2}}{K^{2}}\right] \operatorname{erf}\left(n \sqrt{R_{d}^{\prime}}\right) \\
& -\frac{\xi}{2} \sqrt{\frac{R_{d}^{\prime}}{\pi}}\left\{\sum_{n=-K+3,-K+5}, \cdots,-1\left[\sqrt{R_{d}^{\prime}}\right)\right\}
\end{aligned}
$$




$$
\begin{aligned}
& +\sum_{n=1,3, \cdots, K-1} \exp \left(-n^{2} R_{d}^{\prime}\right) \\
& +\sum_{n=K+1, K+3, \cdots 2 K-3}\left[1-\frac{n-(K-1)}{K}\right] \exp \left(-n^{2} R_{d}^{\prime}\right)
\end{aligned}
$$




\section{APPENDIX D}

\section{DERIVATION OF THE CONDITIONAL ERROR PROBABILITY \\ $P_{E}(\lambda)$ FOR A MASK SYSTEM WITH A FIXED \\ SYMBOL SYNC ERROR}

Consider the situation where the symbol sync is in error by $\epsilon=\lambda T$ sec. The cross-correlator statistic on which a decision is made for the nth transmitted symbol now depends on the received data in the time interval $(n-1) T+\hat{\epsilon} \leq t \leq n T+\hat{\epsilon}$ where $\hat{\epsilon}$ is the sync estimate obtained from the symbol synchronizer. Stated mathematically,

$$
\hat{a}_{n}=Q_{K}\left\{\frac{1}{T} \int_{(n-1) T+\hat{\epsilon}}^{n T+\hat{\epsilon}} y(t) p[t-(n-1) T-\hat{\epsilon}] d t\right\}
$$

where $\hat{a}_{n}$ is the $\underline{n}$ th detected symbol and $y(t) \triangleq s(t, \epsilon)+n(t)$ is the correlation detector input with $s(t, \epsilon)$ defined in (1) of the main text. Making the change of variables $t^{\prime}=t-(n-1) T-\hat{\epsilon}$ in $(D-1)$ and separating the signal and noise correlation components, we get

$$
\hat{a}_{n}=Q_{K}\left\{Q_{s}+Q_{n}\right\}
$$

where

$$
\begin{aligned}
& Q_{s} \triangleq a_{n-1} r_{s}[-T(1-\lambda)]+a_{n} r_{s}(\lambda T)+a_{n+1} r_{s}[T(1+\lambda)] \\
& Q_{n} \triangleq \frac{1}{T} \int_{0}^{T} p_{s}\left(t^{\prime}\right) n\left[t^{\prime}+(n-1) T+\hat{\epsilon}\right] d t^{\prime}
\end{aligned}
$$

and $r_{s}(T)$ is the normalized signal pulse autocorrelation function defined by

$$
r_{S}(\tau)=\frac{1}{T} \int_{T}^{T} p_{S}\left(t^{\prime}\right) p_{S}\left(t^{\prime}-\tau\right) d t^{\prime}=\frac{1}{T} \int_{0}^{T-T} p_{S}\left(t^{\prime}+\tau\right) p_{S}\left(t^{\prime}\right) d t^{\prime}
$$


Actually, if $\lambda$ is known to be positive (or negative); then only two symbols are involved, namely, the nth and one of its adjacent symbols.

The average error probability conditioned on knowing $\lambda$ (assumed positive) is given by

$$
P_{E}(\lambda)=\sum_{j, m} \operatorname{Prob}\left\{\text { error }\left.\right|_{n-1}=j \delta, a_{n}=m \delta\right\} \operatorname{Prob}\left\{a_{n-1}=j \delta, a_{n}=m \delta\right\}
$$

Since the input symbols occur with equal probability and are independent,

$$
\begin{aligned}
P_{E}(\lambda)= & \frac{1}{K^{2}} \sum_{j, m} \operatorname{Prob}\left\{\hat{a}_{n} \neq\left. m \delta\right|_{n}=m \delta, a_{n-1}=j \delta\right\} \\
= & \frac{1}{K^{2}} \sum_{j} \sum_{m= \pm 1, \pm 3, \cdots, \pm(K-3)}\left[\operatorname { P r o b } \left\{Q_{n}+j \delta R_{s}[-T(1-\lambda)\}\right.\right. \\
& \left.+m \delta R_{s}(\lambda T)>(m+1) \delta\right\}+\operatorname{Prob}\left\{Q_{n}+j \delta R_{s}[-T(1-\lambda)]\right. \\
& \left.\left.+m \delta R_{s}(\lambda T)<(m-1) \delta\right\}\right] \\
& +\frac{1}{K^{2}} \sum_{j}\left[\operatorname{Prob}_{j}\left\{Q_{n}+j \delta R_{s}[-T(1-\lambda)]+(K-1) \delta R_{s}(\lambda T)<(K-2) \delta\right\}\right. \\
& \left.+\operatorname{Prob}\left\{Q_{n}+j \delta R_{s}[-T(1-\lambda)]-(K-1) \delta R_{s}(\lambda T)>-(K-2) \delta\right\}\right]
\end{aligned}
$$

Since the random variable $Q_{n}$ is Gaussian with zero mean and variance

$$
\sigma_{\mathrm{Q}}^{2}=\mathrm{E}\left\{\mathrm{Q}_{\mathrm{n}}^{2}\right\}=\frac{\mathrm{N}_{0} \mathrm{~S}}{2 \mathrm{~T}}
$$

\footnotetext{
*Unless otherwise noted, the sums on $m$ and $j$ range over the values $\pm 1, \pm 3$, $\cdots, \pm(K-1)$.
} 
then

$$
\begin{aligned}
& P_{E}(\lambda)=\frac{1}{K^{2}} \sum_{j} \sum_{m= \pm 1, \pm 3, \cdots, \pm(K-3)}\left[\frac { 1 } { 2 } \operatorname { e r f c } \left\{\sqrt { R _ { d } ^ { \prime } } \left[(m+1)-m r_{s}(\lambda T)\right.\right.\right. \\
& \left.\left.-j r_{s}[-T(1-\lambda)]\right]\right\}+\frac{1}{2} \operatorname{erfc}\left\{\sqrt { R _ { d } ^ { 1 } } \left[-(m-1)+m_{s}(\lambda T)\right.\right. \\
& \left.\left.\left.+{ }^{j} r_{s}[-T(1-\lambda)]\right]\right\}\right]+\frac{1}{K^{2}} \sum_{j}\left[\frac { 1 } { 2 } \operatorname { e r f c } \left\{\sqrt { R _ { d } ^ { \prime } } \left[-(K-2)+(K-1) r_{s}(\lambda T)\right.\right.\right. \\
& \left.\left.+\operatorname{jr}_{\mathrm{s}}[-\mathrm{T}(1-\lambda)]\right\}\right\}+\frac{1}{2} \operatorname{erfc}\left\{\sqrt { \mathrm { R } _ { \mathrm { d } } ^ { \prime } } \left[-(\mathrm{K}-2)+(\mathrm{K}-1) \mathrm{r}_{\mathrm{s}}(\lambda \mathrm{T})\right.\right. \\
& \left.\left.\left.-j r_{s}[-T(1-\lambda)]\right]\right\}\right] \\
& =\frac{1}{K^{2}} \sum_{j} \sum_{m= \pm 1, \pm 3, \cdots, \pm(K-3)} \operatorname{erfc}\left\{\sqrt { R _ { d } ^ { 1 } } \left[(m+1)-m_{s}(\lambda T)\right.\right. \\
& \left.\left.-\mathrm{jr}_{\mathrm{s}}[-\mathrm{T}(1-\lambda)]\right]\right\}+\frac{1}{\mathrm{~K}^{2}} \sum_{\mathrm{j}} \operatorname{erfc}\left\{\sqrt{\mathrm{R}_{\mathrm{d}}^{\prime}}[-(\mathrm{K}-2)\right. \\
& \left.\left.+(K-1) r_{s}(\lambda T)-j r_{s}[-T(1-\lambda)]\right]\right\}
\end{aligned}
$$

where $R_{d}^{\prime}$ is defined in (13) of the main text. Combining the two terms of $(D-7)$ into one and considering now the answer for positive and negative $\lambda$, we get

$$
P_{E}(\lambda)=\frac{1}{K^{2}} \sum_{j, l} \operatorname{erfc}\left\{\sqrt{R_{d}^{\prime}}\left[\ell-(\ell-1) r_{s}(|\lambda| T)-j r_{s}(T-|\lambda| T)\right]\right\}
$$

\footnotetext{
"The summation on $\ell$ ranges over the set of values $0, \pm 2, \pm 4, \cdots, \pm(\mathrm{K}-2)$.
} 
For rectangular pulses, i. e., $\mathrm{p}(\mathrm{t})=1,0 \leq \mathrm{t} \leq \mathrm{T}, \mathrm{p}(\mathrm{t})=0$; otherwise,

$$
\mathbf{r}_{S}(T)= \begin{cases}1-\frac{|T|}{T} & |T| \leq T \\ 0 & |T|>T\end{cases}
$$

Thus, for this case $(D-8)$ reduces to

$$
P_{E}(\lambda)=\frac{1}{K^{2}} \sum_{j, \ell} \operatorname{erfc}\left\{\sqrt{R_{d}^{i}}[\ell-(\ell-1)(1-|\lambda|)-j|\lambda|]\right\}
$$




\section{APPENDIX E}

\section{EVALUATION OF THE MOMENTS OF VARIOUS FUNCTIONS \\ ARISING FROM THE PASSAGE OF SIGNAL PLUS \\ NOISE THROUGH A K-LEVEL \\ QUANTIZER}

I. $\mathrm{E}_{\mathrm{n}}\left\{\mathrm{Q}_{\mathrm{K}}(\mathrm{C}+\mathrm{y})\right\}$

Consider the mean of the output of a K-level quantizer with transfer characteristic $Q_{K}(x)$ defined in (7) of the main text, when the input is signal plus noise, $C+y$. Letting y be a Gaussian r.v. with zero mean and standard deviation $\sigma_{y}$, then

$$
E_{n}\left\{Q_{K}(C+y)\right\} \triangleq \operatorname{erf}_{K}\left(\frac{C}{\sqrt{2} \sigma}\right)=\frac{1}{\sqrt{2 \pi} \sigma_{y}} \int_{-\infty}^{\infty} \exp \left(-\frac{y^{2}}{2 \sigma_{y}^{2}}\right) Q_{K}(C+y) d y
$$

where the notation $\operatorname{erf}_{\mathrm{K}}(\mathrm{x})$ is intended to represent a generalization of the standard error function erf $\mathrm{x}$, defined in (18) of the main text to the K-level situation under investigation. When $K=2$ and $\delta=1$, the generalized error function reduces to the standard error function, i.e., erf $f_{2}(x)=$ erf $x$. Substituting (7) of the main text into $(E-1)$ gives

$$
\begin{aligned}
\operatorname{erf}_{\mathrm{K}}\left(\frac{\mathrm{C}}{\sqrt{2} \sigma_{\mathrm{y}}}\right)= & \sum_{\ell= \pm 1, \pm 3, \cdots, \pm(\mathrm{K}-3)} \frac{1}{\sqrt{2 \pi} \sigma_{\mathrm{y}}} \int_{(\ell-1) \delta-\mathrm{C}}^{(\ell+1) \delta-\mathrm{C}} \exp \left(-\frac{\mathrm{y}^{2}}{2 \sigma_{\mathrm{y}}^{2}}\right) \ell \delta \mathrm{dy} \\
& +\frac{1}{\sqrt{2 \pi} \sigma_{\mathrm{y}}} \int_{(\mathrm{K}-2) \delta-\mathrm{C}}^{\infty} \exp \left(-\frac{\mathrm{y}^{2}}{2 \sigma_{\mathrm{y}}^{2}}\right)(\mathrm{K}-1) \delta \mathrm{dy} \\
& +\frac{1}{\sqrt{2 \pi} \sigma} \int_{-\infty}^{-(\mathrm{K}-2) \delta-\mathrm{C}} \exp \left(-\frac{\mathrm{y}^{2}}{2 \sigma_{\mathrm{y}}^{2}}\right)[-(\mathrm{K}-1) \delta] \mathrm{dy}
\end{aligned}
$$


or

$$
\begin{aligned}
\operatorname{erf}_{\mathrm{K}}\left(\frac{\mathrm{C}}{\sqrt{2} \sigma_{\mathrm{y}}}\right)= & \sum_{\ell= \pm 1, \pm 3, \cdots, \pm(\mathrm{K}-3)} \frac{1}{2} \ell \delta\left\{\operatorname{erf}\left[\frac{(\ell+1) \delta-\mathrm{C}}{\sqrt{2} \sigma_{\mathrm{y}}}\right]\right. \\
& +\operatorname{erf}\left[\frac{-(\ell-1) \delta+\mathrm{C}}{\sqrt{2} \sigma_{\mathrm{y}}}\right]-\frac{1}{2}(\mathrm{~K}-1) \delta \operatorname{erf}\left[\frac{(\mathrm{K}-2) \delta-\mathrm{C}}{\sqrt{2} \sigma_{\mathrm{y}}}\right] \\
& \left.+\frac{1}{2}(\mathrm{~K}-1) \delta \operatorname{erf}\left[\frac{(\mathrm{K}-2) \delta+\mathrm{C}}{\sqrt{2} \sigma_{\mathrm{y}}}\right]\right\}
\end{aligned}
$$

Finally, factoring out $\delta / 2$ gives

$$
\begin{aligned}
\operatorname{erf}_{\mathrm{K}}\left(\frac{\mathrm{C}}{\sqrt{2} \sigma_{\mathrm{y}}}\right)= & \frac{\delta}{2}\left\{(\mathrm{~K}-1)\left[\operatorname{erf}\left(\frac{\mathrm{C}+(\mathrm{K}-2) \delta}{\sqrt{2 \sigma_{\mathrm{y}}}}\right)+\operatorname{erf}\left(\frac{\mathrm{C}-(\mathrm{K}-2) \delta}{\sqrt{2 \sigma_{\mathrm{y}}}}\right)\right]\right. \\
& +\sum_{\ell= \pm 1, \pm 3, \cdots, \pm(\mathrm{K}-3)}\left[\operatorname{erf}\left(\frac{\mathrm{C}-(\ell-1) \delta}{\sqrt{2} \sigma_{\mathrm{y}}}\right)\right. \\
& \left.\left.-\operatorname{erf}\left(\frac{\mathrm{C}-(\ell+1) \delta}{\sqrt{2} \sigma_{\mathrm{y}}}\right)\right]\right\}
\end{aligned}
$$

II. $\quad E_{n}\left\{Q_{K}^{2}(C+y)\right\}$

We now consider the mean of the $K$-level quantizer output squared. If $\mathrm{K}=2$ and $\delta=1$, then the quantizer becomes a signum device whose output when squared has value unity regardless of the value of the input. Thus, the generalization of this result for the $K$-level quantizer is 


$$
E_{n}\left\{Q_{K}^{2}(C+y)\right\} \triangleq 1_{K}^{(2)}\left(\frac{C}{\sqrt{2} \sigma_{y}}\right)=\frac{1}{\sqrt{2 \pi} \sigma_{y}} \int_{-\infty}^{\infty} \exp \left(-\frac{y^{2}}{2 \sigma_{y}^{2}}\right) Q_{K}^{2}(\dot{C}+y) d y
$$

The superscript notation on the function $1_{K}^{(2)}(x)$ refers to the fact that we are considering the mean of the square of $Q_{K}(x)$. Also, from the above, for $K=2$ and $\delta=1$, we have that $1_{\mathrm{K}}^{(2)}(x)=1$. Squaring (7) of the main text and substituting the result in (E-4) gives

$$
\begin{aligned}
& 1_{\mathrm{K}}^{(2)}\left(\frac{\mathrm{C}}{\sqrt{2} \sigma_{\mathrm{y}}}\right)=\sum_{\ell= \pm 1, \pm 3, \cdots, \pm(\mathrm{K}-3)} \frac{1}{\sqrt{2 \pi} \sigma_{\mathrm{y}}} \int_{(\ell-1) \delta-C}^{(\ell+1) \delta-C} \exp \left(-\frac{\mathrm{y}^{2}}{2 \sigma_{\mathrm{y}}^{2}}\right)(\ell \delta)^{2} \mathrm{dy} \\
& +\frac{1}{\sqrt{2 \pi} \sigma} \int_{(K-2) \delta-C}^{\infty} \exp \left(-\frac{y^{2}}{2 \sigma_{y}^{2}}\right)[(K-1) \delta]^{2} d y \\
& +\frac{1}{\sqrt{2 \pi} \sigma} \int_{-\infty}^{-(K-2) \delta-C} \exp \left(-\frac{y^{2}}{2 \sigma_{y}^{2}}\right)[-(K-1) \delta]^{2} d y
\end{aligned}
$$

or by inspection,

$$
\begin{aligned}
1_{\mathrm{K}}^{(2)}\left(\frac{\mathrm{C}}{\sqrt{2} \sigma_{\mathrm{y}}}\right)= & \frac{\delta^{2}}{2}\left\{(\mathrm{~K}-1)^{2}\left[2-\operatorname{erf}\left(\frac{\mathrm{C}+(\mathrm{K}-2) \delta}{\sqrt{2} \sigma_{\mathrm{y}}}\right)+\operatorname{erf}\left(\frac{\mathrm{C}-(\mathrm{K}-2) \delta}{\sqrt{2} \sigma_{\mathrm{y}}}\right)\right]\right. \\
& +\sum_{\ell= \pm 1, \pm 3, \cdots \pm(\mathrm{K}-3)} \ell^{2}\left[\operatorname{erf}\left(\frac{\mathrm{C}-(\ell-1) \delta}{\sqrt{2} \sigma_{\mathrm{y}}}\right)\right. \\
& \left.\left.-\operatorname{erf}\left(\frac{\mathrm{C}-(\ell+1) \delta}{\sqrt{2} \sigma_{\mathrm{y}}}\right)\right]\right\}
\end{aligned}
$$


It is a simple matter now to generalize the above to the case where the $\mathrm{K}-l e v e l$ quantizer output is raised to an arbitrary power. Thus,

$$
E_{n}\left\{Q_{K}^{m}(C+y)\right\} \triangleq \begin{cases}l_{K}^{(m)}\left(\frac{C}{\sqrt{2} \sigma_{y}}\right) & m \text { even } \\ e r f_{K}^{(m)}\left(\frac{C}{\sqrt{2} \sigma_{y}}\right) & \text { m odd }\end{cases}
$$

where

$$
\begin{aligned}
& \mathbf{I}_{\mathrm{K}}^{(\mathrm{m})}\left(\frac{\mathrm{C}}{\sqrt{2} \sigma_{\mathrm{y}}}\right)=\frac{\delta^{\mathrm{m}}}{2}\left\{(\mathrm{~K}-1)^{\mathrm{m}}\left[2-\operatorname{erf}\left(\frac{C+(\mathrm{K}-2) \delta}{\sqrt{2} \sigma_{\mathrm{y}}}\right)+\operatorname{erf}\left(\frac{C-(\mathrm{K}-2) \delta}{\sqrt{2} \sigma_{\mathrm{y}}}\right)\right]\right. \\
& +\sum_{\ell= \pm 1, \pm 3, \cdots, \pm(\mathrm{K}-3)} \ell^{m}\left[\operatorname{erf}\left(\frac{\mathrm{C}-(\ell-1)}{\sqrt{2 \sigma_{\mathrm{y}}}}\right)\right. \\
& \left.\left.-\operatorname{erf}\left(\frac{C-(\ell+1) \delta}{\sqrt{2 \sigma_{y}}}\right)\right]\right\} \\
& \operatorname{erf}_{K}^{(m)}\left(\frac{C}{\sqrt{2} \sigma_{y}}\right)=\frac{\delta^{m}}{2}\left\{(K-1)^{m}\left[\operatorname{erf}\left(\frac{C+(K-2) \delta}{\sqrt{2} \sigma_{y}}\right)+\operatorname{erf}\left(\frac{C-(K-2) \delta}{\sqrt{2} \sigma_{y}}\right)\right]\right. \\
& +\sum_{\ell= \pm 1, \pm 3, \cdots, \pm(K-3)} \ell^{m}\left[\operatorname{erf}\left(\frac{C-(\ell-1) \delta}{\sqrt{2} \sigma_{y}}\right)\right. \\
& \left.\left.-\operatorname{erf}\left(\frac{C-(\ell+1) \delta}{\sqrt{2} \sigma_{y}}\right)\right]\right\}
\end{aligned}
$$


and $\operatorname{erf}{ }_{K}^{(1)}(x) \triangleq \operatorname{erf}_{K}(x)$. Note that for $K=2, \delta=1$, and arbitrary $m$,

$$
\begin{aligned}
& \operatorname{erf}_{K}^{(m)}(x)=\operatorname{erf} x \\
& \left(1_{\mathrm{K}}^{(\mathrm{m})}(\mathrm{x})=1\right. \\
& \text { III. } E_{n}\left\{z \operatorname{erf}_{K}\left(\frac{C+z}{\sqrt{2} \sqrt{\sigma_{y}^{2}-\sigma_{z}^{2}}}\right)\right\}
\end{aligned}
$$

$$
\begin{aligned}
& E_{n}\left\{z \operatorname{erf}_{K}\left(\frac{C+z}{\sqrt{2} \sqrt{\sigma_{y}^{2}-\sigma_{z}^{2}}}\right)\right\}=\frac{\delta}{2}\left\{( K - 1 ) \left[E\left\{z \operatorname{erf}\left(\frac{C+z+(K-2) \delta}{\sqrt{2} \sqrt{\sigma_{y}^{2}-\sigma_{z}^{2}}}\right)\right\}\right.\right. \\
& +E\left\{z \operatorname{erf}\left(\frac{C+z-(K-2) \delta}{\sqrt{2} \sqrt{\sigma_{y}^{2}-\sigma_{z}^{2}}}\right)\right\} \\
& +\sum_{\ell= \pm 1, \pm 3, \cdots, \pm(K-3)} \ell\left[\mathrm{E}\left\{\mathrm{erf}\left(\frac{\mathrm{C}+\mathrm{z}-(\ell-1) \delta}{\sqrt{2} \sqrt{\sigma_{y}^{2}-\sigma_{z}^{2}}}\right)\right\}\right. \\
& \left.\left.-E\left\{z \operatorname{erf}\left(\frac{C+z-(\ell+1) \delta}{\sqrt{2} \sqrt{\sigma_{y}^{2}-\sigma_{z}^{2}}}\right)\right\}\right]\right\}
\end{aligned}
$$

$$
\begin{array}{ll}
\vdots & \\
\vdots & \\
\ddots &
\end{array}
$$

Applying $(A-11)$ to $(A-9)$ results in

$$
E_{n}\left\{z \operatorname{erf}_{K}\left(\frac{C+z}{\sqrt{2 \sqrt{\sigma_{y}^{2}-\sigma_{z}^{2}}}}\right)\right\}=\sqrt{\frac{2}{\pi}} \frac{\sigma^{2}}{\sigma_{y}} \exp \left(-\frac{C^{2}}{2 \sigma_{y}^{2}}\right)
$$


where

$$
\begin{aligned}
\exp _{\mathrm{K}}\left(-\frac{\mathrm{C}^{2}}{2 \sigma_{\mathrm{y}}^{2}}\right) \triangleq & \frac{\delta}{2}\left\{(\mathrm{~K}-1)\left[\exp \left\{-\frac{(\mathrm{C}+(\mathrm{K}-2) \delta)^{2}}{2 \sigma_{\mathrm{y}}^{2}}\right\}+\exp \left\{-\frac{(\mathrm{C}-(\mathrm{K}-2) \delta)^{2}}{2 \sigma_{\mathrm{y}}^{2}}\right\}\right]\right\} \\
+ & \sum_{\ell= \pm 1, \pm 3, \cdots, \pm(\mathrm{K}-3)}\left(\exp \left\{-\frac{(\mathrm{C}-(\ell-1) \delta)^{2}}{2 \sigma_{\mathrm{y}}^{2}}\right\}\right. \\
& \left.\left.-\exp \left\{-\frac{(\mathrm{C}-(\ell+1) \delta)^{2}}{2 \sigma_{\mathrm{y}}^{2}}\right\}\right]\right\}
\end{aligned}
$$

Again, for $K=2$ and $\delta=1$,

$$
\exp _{K}\left(-\frac{C^{2}}{2 \sigma_{y}^{2}}\right)=\exp \left(-\frac{C^{2}}{2 \sigma_{y}^{2}}\right)
$$

More generally, for m odd,

$$
E_{n}\left\{z \operatorname{erf}_{K}^{(m)}\left(\frac{C+z}{\sqrt{2} \sqrt{\sigma_{y}^{2}-\sigma_{z}^{2}}}\right)=\sqrt{\frac{2}{\pi}} \frac{\sigma_{z}^{2}}{\sigma_{y}} \exp _{K}^{(m)}\left(-\frac{C^{2}}{2 \sigma_{y}^{2}}\right)\right.
$$


where

$$
\begin{aligned}
\exp _{\mathrm{K}}^{(\mathrm{m})}\left(-\frac{\mathrm{C}^{2}}{2 \sigma_{\mathrm{y}}^{2}}\right) \triangleq & \frac{\delta^{\mathrm{m}}}{2}\left\{(\mathrm{~K}-1)^{\mathrm{m}}\left[\exp \left\{-\frac{(\mathrm{C}+(\mathrm{K}-2) \delta)^{2}}{2 \sigma_{\mathrm{y}}^{2}}\right\}+\exp \left\{-\frac{(\mathrm{C}-(\mathrm{K}-2) \delta)^{2}}{2 \sigma_{\mathrm{y}}^{2}}\right\}\right]\right. \\
& +\sum_{\ell= \pm 1, \pm 3, \cdots, \pm(\mathrm{K}-3)} \ell^{\mathrm{m}\left[\exp \left\{-\frac{(\mathrm{C}-(\ell-1) \delta)^{2}}{2 \sigma_{\mathrm{y}}^{2}}\right\}\right.} \\
& \left.\left.-\exp \left\{-\frac{(\mathrm{C}-(\ell+1) \delta)^{2}}{2 \sigma_{\mathrm{y}}^{2}}\right\}\right]\right\}
\end{aligned}
$$

and

$$
\exp _{K}^{(1)}\left(-\frac{C^{2}}{2 \sigma_{y}^{2}}\right) \triangleq \exp _{K}\left(-\frac{C^{2}}{2 \sigma_{y}^{2}}\right)
$$

Also, for $K=2, \delta=1$, and arbitrary $m$,

$$
\exp _{K}^{(m)}\left(-\frac{C^{2}}{2 \sigma_{y}^{2}}\right)=\exp \left(-\frac{C^{2}}{2 \sigma_{y}^{2}}\right)
$$

IV. $E_{n}\left\{z 1_{K}^{(2)}\left(\frac{C+z}{\sqrt{2} \sqrt{\sigma_{y}^{2}-\sigma_{z}^{2}}}\right)\right\}$

From (E-6) and the steps leading up to (E-10) we can immediately write $\therefore$ that

$$
\mathrm{E}_{\mathrm{n}}\left\{\mathrm{z} 1_{\mathrm{K}}^{(2)}\left(\frac{\mathrm{C}+\mathrm{z}}{\sqrt{2} \sqrt{\sigma_{\mathrm{y}}^{2}-\sigma_{\mathrm{z}}^{2}}}\right) \Leftrightarrow \sqrt{\frac{2}{\pi}} \frac{\sigma^{2}}{\sigma_{\mathrm{y}}} 0_{\mathrm{K}}^{(2)}\left(-\frac{\mathrm{C}^{2}}{2 \sigma_{\mathrm{y}}^{2}}\right)\right.
$$


where

$$
\begin{aligned}
0_{\mathrm{K}}^{(2)}\left(-\frac{\mathrm{C}^{2}}{2 \sigma_{\mathrm{y}}^{2}}\right) \triangleq & \frac{\delta^{2}}{2}\left\{(\mathrm{~K}-1)^{2}\left[-\exp \left\{-\frac{(\mathrm{C}+(\mathrm{K}-2) \delta)^{2}}{2 \sigma_{\mathrm{y}}^{2}}\right\}+\exp \left\{-\frac{\left.(\mathrm{C}-(\mathrm{K}-2) \delta)^{2}\right)}{2 \sigma_{\mathrm{y}}^{2}}\right\}\right]\right. \\
& +\sum_{\ell= \pm 1, \pm 3, \cdots, \pm(\mathrm{K}-3)} \ell^{2}\left[\exp \left\{-\frac{(\mathrm{C}-(\ell-1) \delta)^{2}}{2 \sigma_{\mathrm{y}}^{2}}\right\}\right. \\
& \left.\left.-\exp \left\{-\frac{(\mathrm{C}-(\ell+1) \delta)^{2}}{2 \sigma_{\mathrm{y}}^{2}}\right\}\right]\right\}
\end{aligned}
$$

For $\mathrm{K}=2$ and $\delta=1$,

$$
0_{K}^{(2)}\left(-\frac{C^{2}}{2 \sigma_{y}^{2}}\right)=0
$$

More generally, for $m$ even,

$$
E_{n}\left\{z 1_{K}^{(m)}\left(\frac{C+z}{\sqrt{2} \sqrt{\sigma_{y}^{2}-\sigma_{z}^{2}}}\right)\right\}=\sqrt{\frac{2}{\pi} \frac{\sigma_{z}^{2}}{\sigma_{y}}} 0_{K}^{(m)}\left(-\frac{C^{2}}{2 \sigma_{y}^{2}}\right)
$$

where

$$
\begin{aligned}
0_{\mathrm{K}}^{(\mathrm{m})}\left(-\frac{\mathrm{C}^{2}}{2 \sigma_{\mathrm{y}}^{2}}\right)= & \frac{\delta^{\mathrm{m}}}{2}\left\{(\mathrm{~K}-1)^{\mathrm{m}}\left[-\exp \left\{-\frac{(\mathrm{C}+(\mathrm{K}-2) \delta)^{2}}{2 \sigma_{\mathrm{y}}^{2}}\right\}+\exp \left\{-\frac{(\mathrm{C}-(\mathrm{K}-2) \delta)^{2}}{2 \sigma_{\mathrm{y}}^{2}}\right\}\right]\right. \\
& +\sum_{\ell= \pm 1, \pm 3, \cdots, \pm(\mathrm{K}-3)} \ell^{\mathrm{m}}\left[\exp \left\{-\frac{(\mathrm{C}-(\ell-1) \delta)^{2}}{2 \sigma_{\mathrm{y}}^{2}}\right\}\right.
\end{aligned}
$$




$$
\left.\left.-\exp \left\{-\frac{(C-(\ell+1) \delta)^{2}}{2 \sigma_{y}^{2}}\right\}\right]\right\}
$$

Also, for $\mathrm{K}=2, \delta=1$ and arbitrary $\mathrm{m}$

$$
\begin{array}{r}
0_{K}^{(m)}\left(-\frac{C^{2}}{2 \sigma_{y}^{2}}\right)=0 \\
\text { V. } E_{n}\left\{z^{2} \operatorname{erf}_{K}\left(\frac{C+z}{\sqrt{2 \sqrt{\sigma_{y}^{2}-\sigma_{z}^{2}}}}\right)\right\}
\end{array}
$$

From $(E-3)$ and the relation

$E\left\{x^{2} \operatorname{erf}(a+b x)\right\}=\sigma_{x}^{2}\left[\operatorname{erf}\left(\frac{a}{\sqrt{1+2 b^{2} \sigma_{x}^{2}}}\right)-\frac{4 a\left(b \sigma_{x}\right)^{2}}{\sqrt{\pi}\left(1+2 b^{2} \sigma_{x}^{2}\right)^{3 / 2}} \exp \left(\frac{-a^{2}}{1+2 b^{2} \sigma_{x}^{2}}\right)\right.$

we see that

$$
\begin{aligned}
E_{n}\left\{z^{2} \operatorname{erf}_{K}\left(\frac{C+z}{\sqrt{2} \sqrt{\sigma_{y}^{2}-\sigma_{z}^{2}}}\right)\right\}= & \frac{\delta}{2} \sigma_{z}^{2}\left\{( K - 1 ) \left[\operatorname{erf}\left(\frac{C+(K-2) \delta}{\sqrt{2} \sigma_{y}}\right)+\operatorname{erf}\left(\frac{C-(K-2) \delta}{\sqrt{2} \sigma_{y}}\right)\right.\right. \\
& -\frac{\xi}{\sqrt{\pi}}\left(\frac{C+(K-2) \delta}{\sqrt{2} \sigma}\right) \exp \left\{-\frac{(C+(K-2) \delta)^{2}}{2 \sigma_{y}^{2}}\right\} \\
& \left.-\frac{\xi}{\sqrt{\pi}}\left(\frac{C-(K-2) \delta}{\sqrt{2} \sigma_{y}}\right) \exp \left\{-\frac{(C-(K-2) \delta)^{2}}{2 \sigma_{y}^{2}}\right\}\right]
\end{aligned}
$$




$$
\begin{aligned}
& +\sum_{\ell= \pm 1, \pm 3, \cdots, \pm(\mathrm{K}-3)} \ell\left[\operatorname{erf}\left(\frac{\mathrm{C}-(\ell-1) \delta}{\sqrt{2} \sigma_{\mathrm{y}}}\right)\right. \\
& -\operatorname{erf}\left(\frac{\mathrm{C}-(\ell+1) \delta}{\sqrt{2} \sigma_{\mathrm{y}}}\right) \\
& -\frac{\xi}{\sqrt{\pi}}\left(\frac{\mathrm{C}-(\ell-1) \delta}{\sqrt{2} \sigma_{\mathrm{y}}}\right) \exp \left\{-\frac{(\mathrm{C}-(\ell-1) \delta)^{2}}{2 \sigma_{\mathrm{y}}^{2}}\right\} \\
& \left.\left.+\frac{\xi}{\sqrt{\pi}}\left(\frac{\mathrm{C}-(\ell+1) \delta}{\sqrt{2} \sigma_{\mathrm{y}}}\right) \exp \left\{-\frac{(\mathrm{C}-(\ell+1) \delta)^{2}}{2 \sigma_{\mathrm{y}}^{2}}\right)\right]\right\}
\end{aligned}
$$

$(\mathrm{E}-23)$

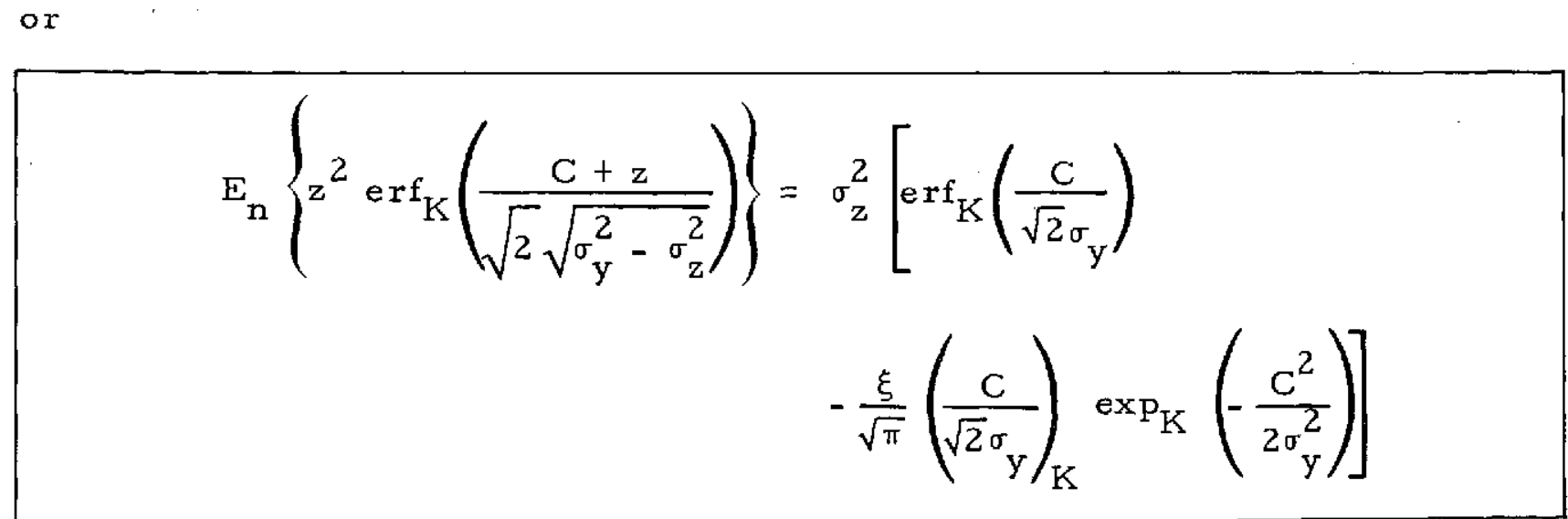

$(E-24)$

where $\operatorname{erf}_{K}(x)$ is defined in (E-3) and 


$$
\begin{aligned}
& \left(\frac{C}{\sqrt{2} \sigma_{y}}\right)_{K} \exp _{K}\left(-\frac{C^{2}}{2 \sigma_{y}^{2}}\right) \triangleq \frac{\delta}{2}\left\{(K-1)\left(\frac{C+(K-2) \delta}{\sqrt{2} \sigma_{y}}\right) \exp \left\{-\frac{(C+(K-2) \delta)^{2}}{2 \sigma_{y}^{2}}\right\}\right. \\
& +(K-1)\left(\frac{C-(K-2) \delta}{\sqrt{2} \sigma_{y}}\right) \exp \left\{-\frac{(C-(K-2) \delta)^{2}}{2 \sigma_{y}^{2}}\right\}
\end{aligned}
$$

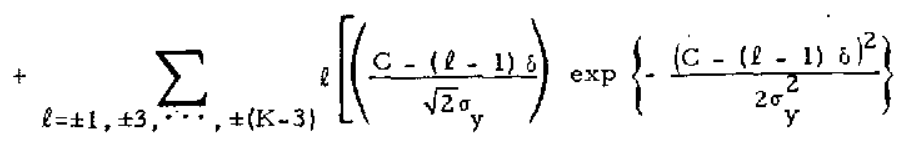

$$
\begin{aligned}
& \left.\left.-\left(\frac{C-(\ell+1) \delta}{\sqrt{2} \sigma_{y}}\right) \exp \left\{-\frac{(C-(\ell+1) \delta)^{2}}{2 \sigma_{y}^{2}}\right\}\right]\right\}
\end{aligned}
$$

For $\mathrm{K}=2$ and $\delta=1$,

$$
\left(\frac{\mathrm{C}}{\sqrt{2} \sigma_{\mathrm{y}}}\right)_{\mathrm{K}} \exp _{\mathrm{K}}\left(-\frac{\mathrm{C}^{2}}{2 \sigma_{\mathrm{y}}^{2}}\right)=\frac{\mathrm{C}}{\sqrt{2} \sigma_{\mathrm{y}}} \exp \left(-\frac{\mathrm{C}^{2}}{2 \sigma_{\mathrm{y}}^{2}}\right)
$$

VI. $E_{n}\left\{z^{2} 1_{K}^{(2)}\left(\frac{c+z}{\sqrt{2} \sqrt{\sigma_{y}^{2}-\sigma_{z}^{2}}}\right)\right\}$

From (E-6) and the steps leading up to (E-24) we can immediately write that

$$
\begin{aligned}
& E_{n}\left\{z^{2} 1_{K}^{(2)}\left(\frac{C+z}{\sqrt{2 \sqrt{\sigma_{y}^{2}-\sigma_{z}^{2}}}}\right)\right\} \triangleq \sigma_{z_{K}}^{2}\left(\frac{C}{\sqrt{2} \sigma_{y}}\right) \\
& =\sigma_{z}^{2}\left[1_{K}^{(2)}\left(\frac{C}{\sqrt{2} \sigma_{y}}\right)-\frac{\xi}{\sqrt{\pi}}\left(\frac{C}{\sqrt{2} \sigma_{y}}\right)_{K} 0_{K}^{(2)}\left(-\frac{C^{2}}{2 \sigma_{y}^{2}}\right)\right]
\end{aligned}
$$


where $1_{K}^{(2)}(x)$ is defined in $(E-6)$ and

$$
\begin{aligned}
\left(\frac{\mathrm{C}}{\sqrt{2} \sigma_{\mathrm{y}}}\right)_{\mathrm{K}} 0_{\mathrm{K}}^{(2)}\left(-\frac{\mathrm{C}^{2}}{2 \sigma_{\mathrm{y}}^{2}}\right) \triangleq & \frac{\delta^{2}}{2}\left\{-(\mathrm{K}-1)^{2}\left(\frac{\mathrm{C}+(\mathrm{K}-2) \delta}{\sqrt{2} \sigma_{\mathrm{y}}}\right) \exp \left\{-\frac{\left.\mid \mathrm{C}+(\mathrm{K}-2) \delta)^{2}\right\}}{2 \sigma_{\mathrm{y}}^{2}}\right\}\right. \\
& +(\mathrm{K}-1)^{2}\left(\frac{\mathrm{C}-(\mathrm{K}-2) \delta}{\sqrt{2} \sigma_{\mathrm{y}}}\right) \exp \left\{-\frac{\left.(\mathrm{C}-(\mathrm{K}-2) \delta)^{2}\right)}{2 \sigma_{\mathrm{y}}^{2}} \mid\right. \\
& +(\ell= \pm 1, \pm 3, \cdots, \pm(\mathrm{K}-3) \\
& \left.\left.-\left(\frac{\mathrm{C}-(\ell+1) \delta}{\sqrt{2} \sigma_{\mathrm{y}}}\right) \exp \left(-\frac{\left.\left.(\mathrm{C}-(\ell+1) \delta)^{2}\right)\right]}{2 \sigma_{\mathrm{y}}^{2}}\right)\right]\right\}
\end{aligned}
$$

(E-28) 


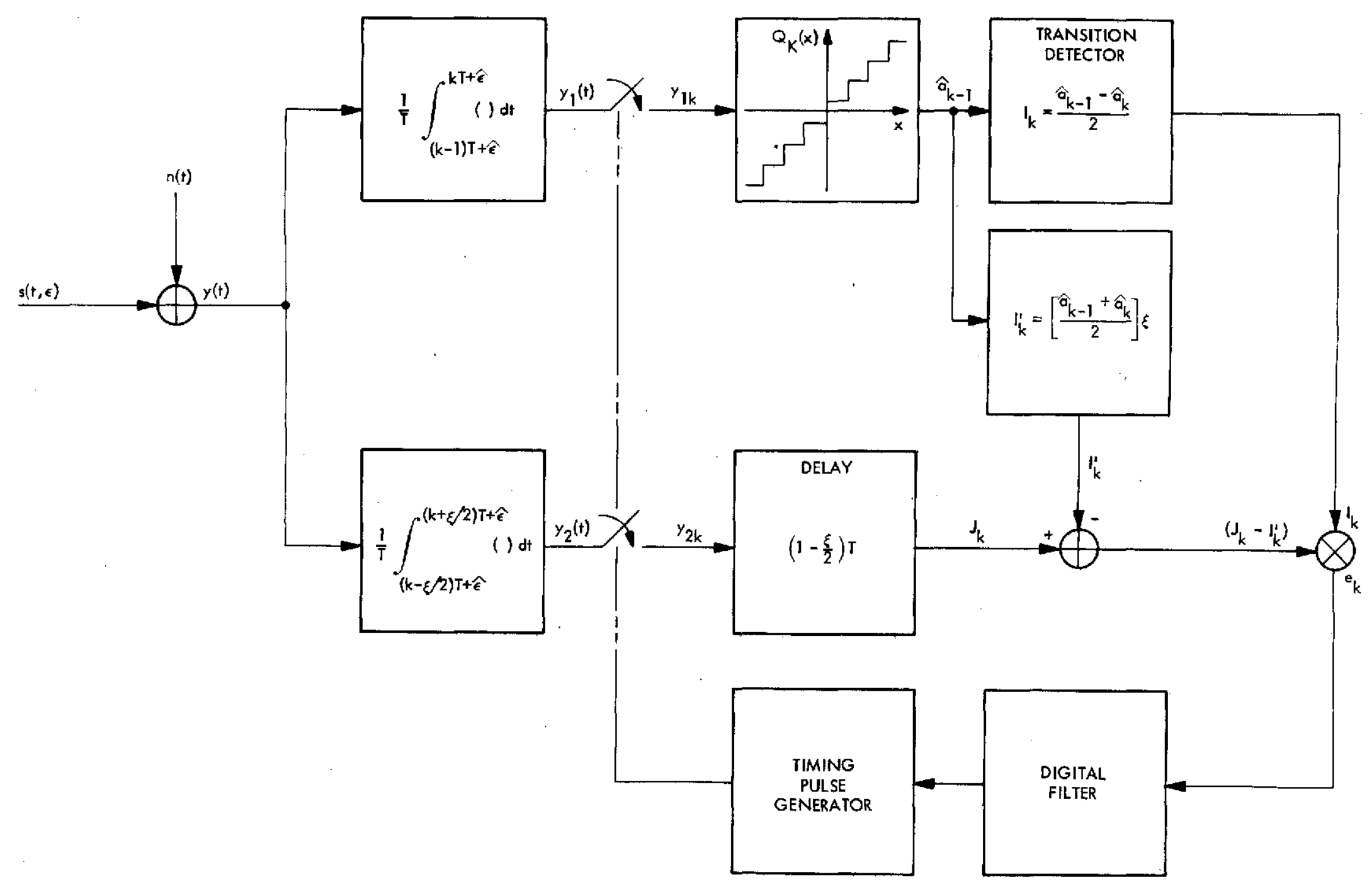




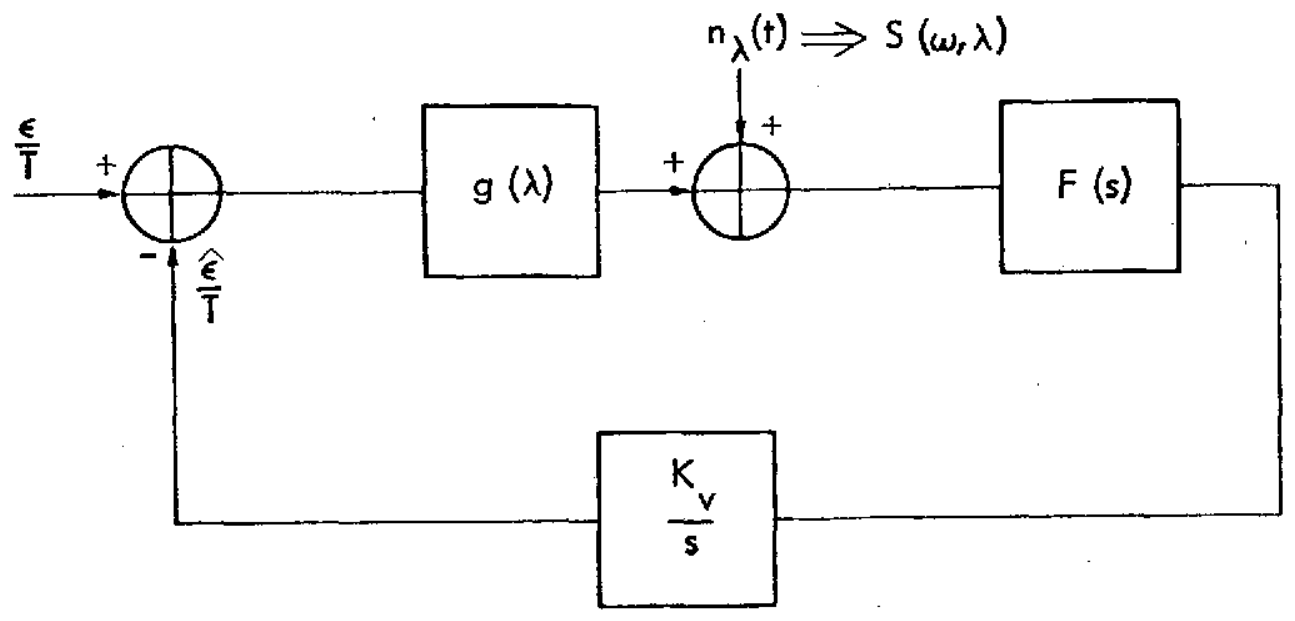

Fig. 2. Equivalent Data-Transition Tracking Loop Model 


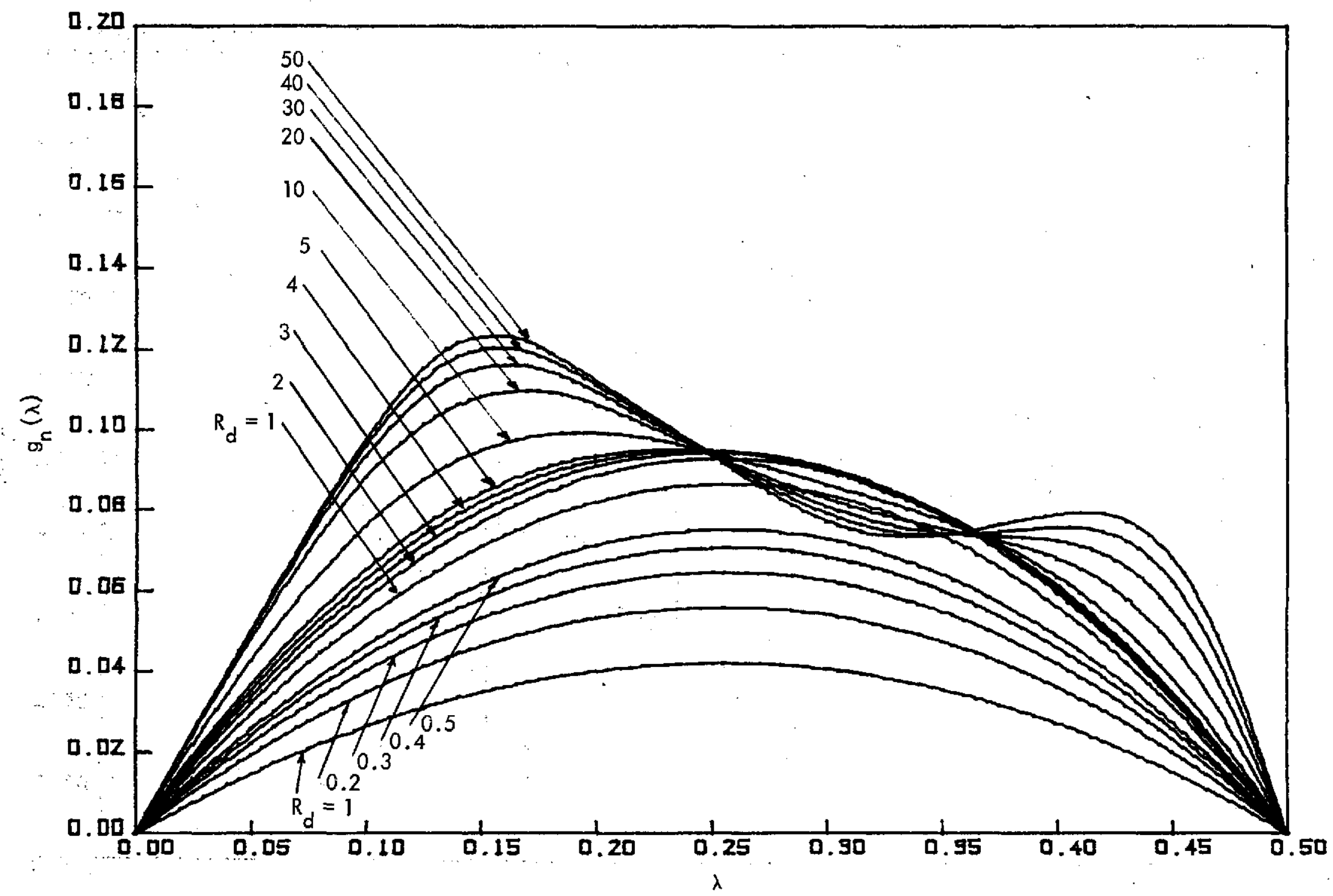

Fig. 3a. Normalized Loop Nonlinearity vs Normalized Symbol Sync Error; $\xi=1$ 


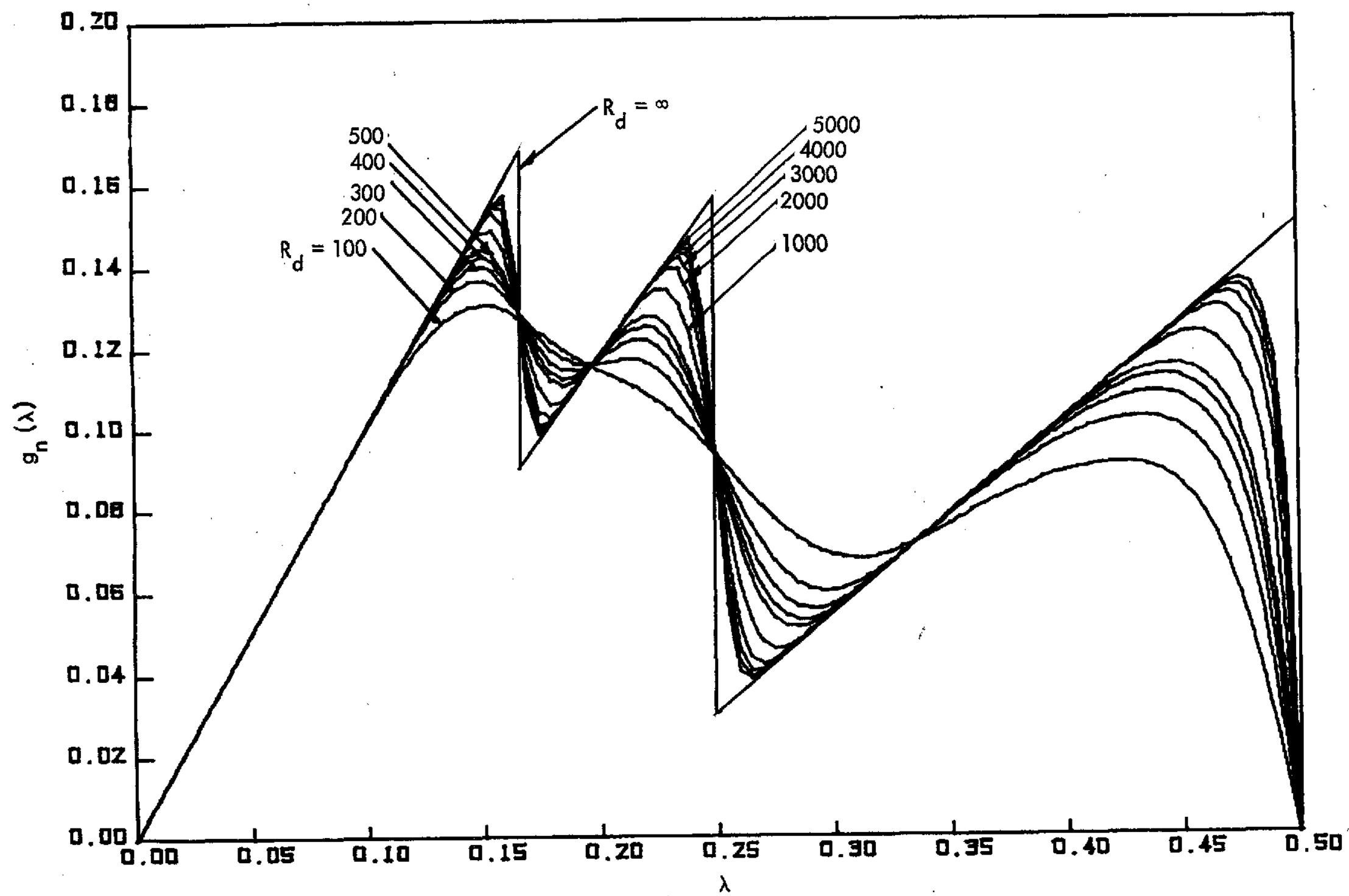

Fig. 3b. Normalized Loop Nonlinearity vs Normalized Symbol Sync Error; $\xi=1$ 


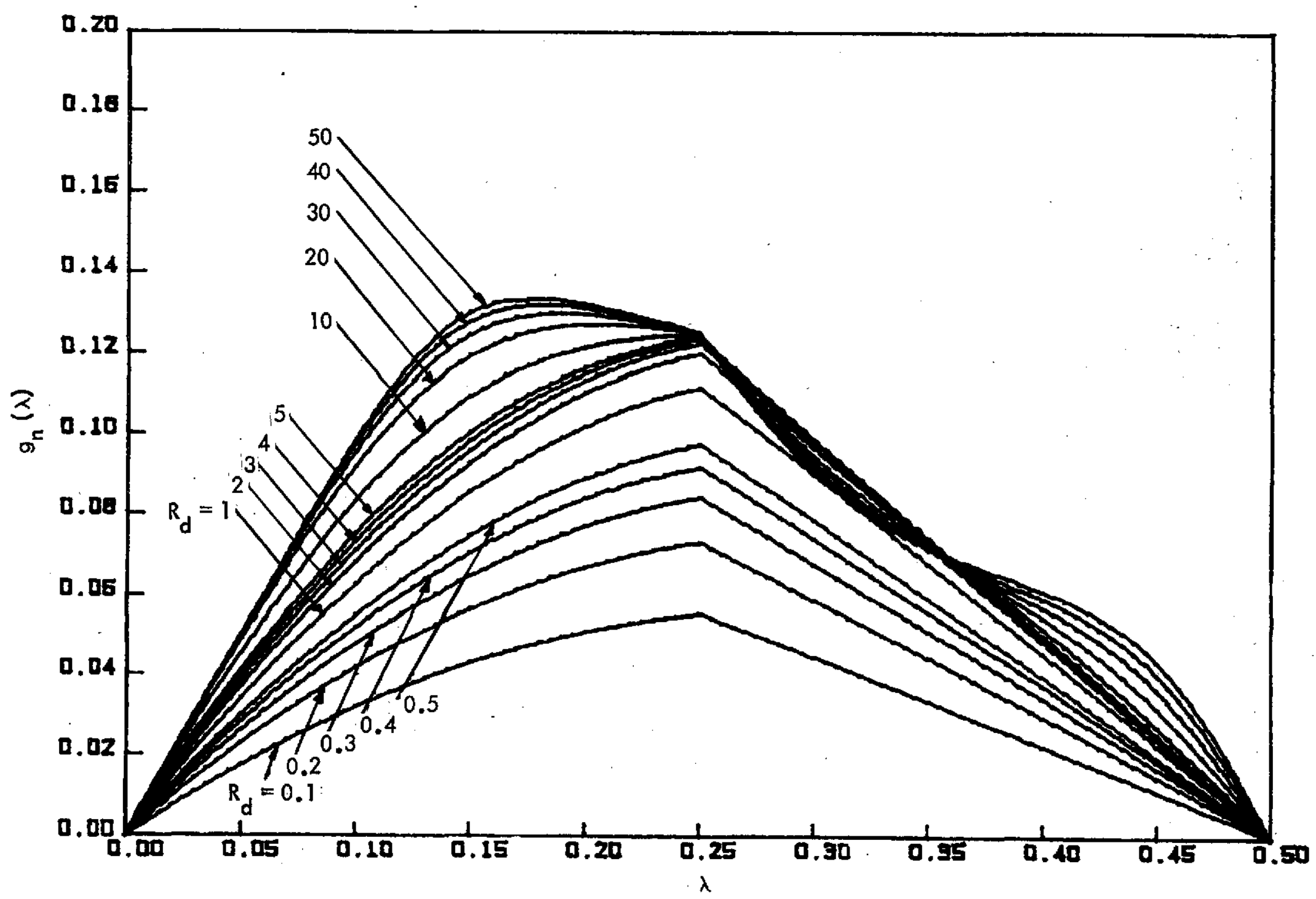




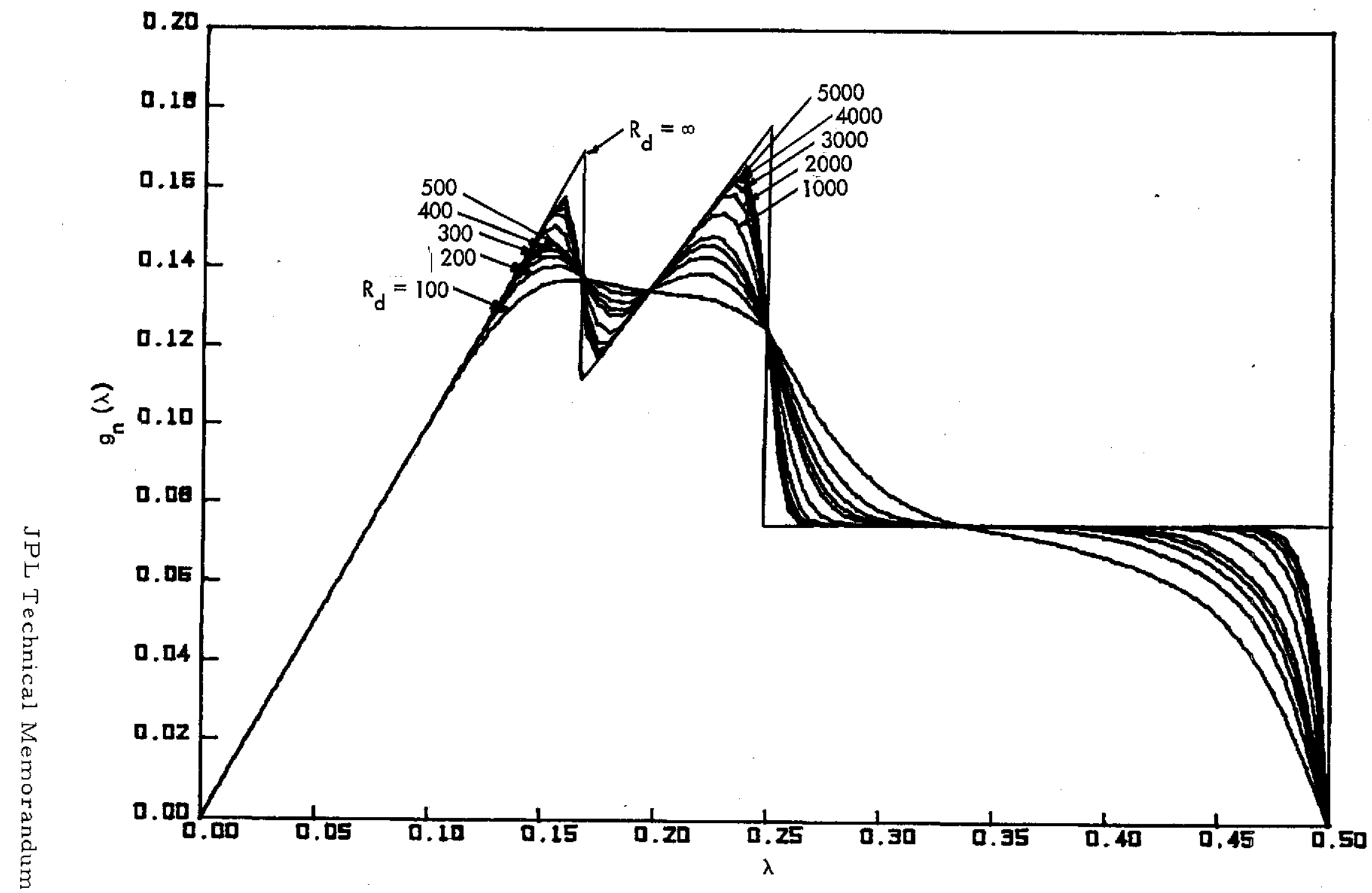

Fig. 4b. Normalized Loop Nonlinearity vs Normalized Symbol Sync Error; $\xi=0.5$ 


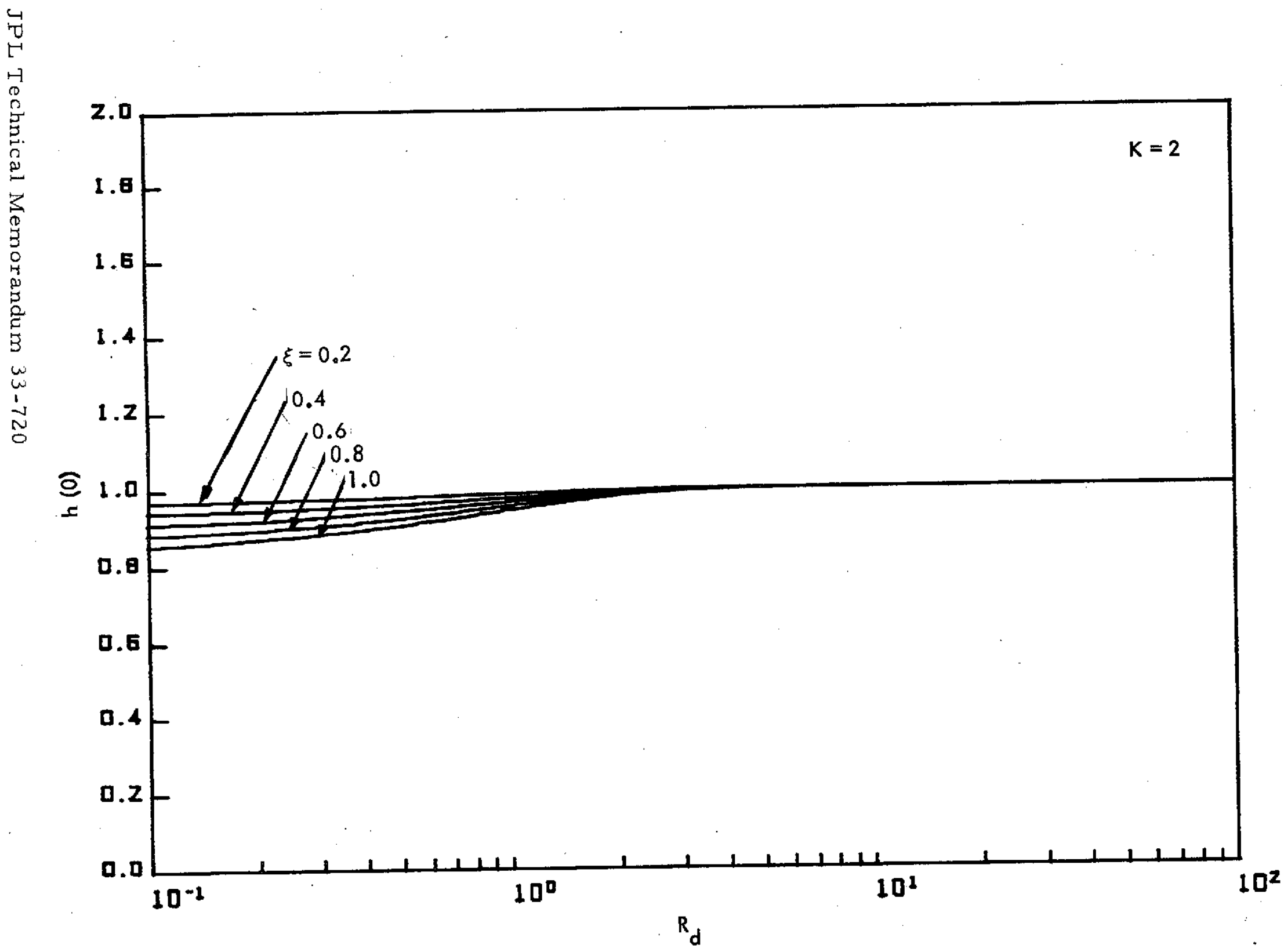




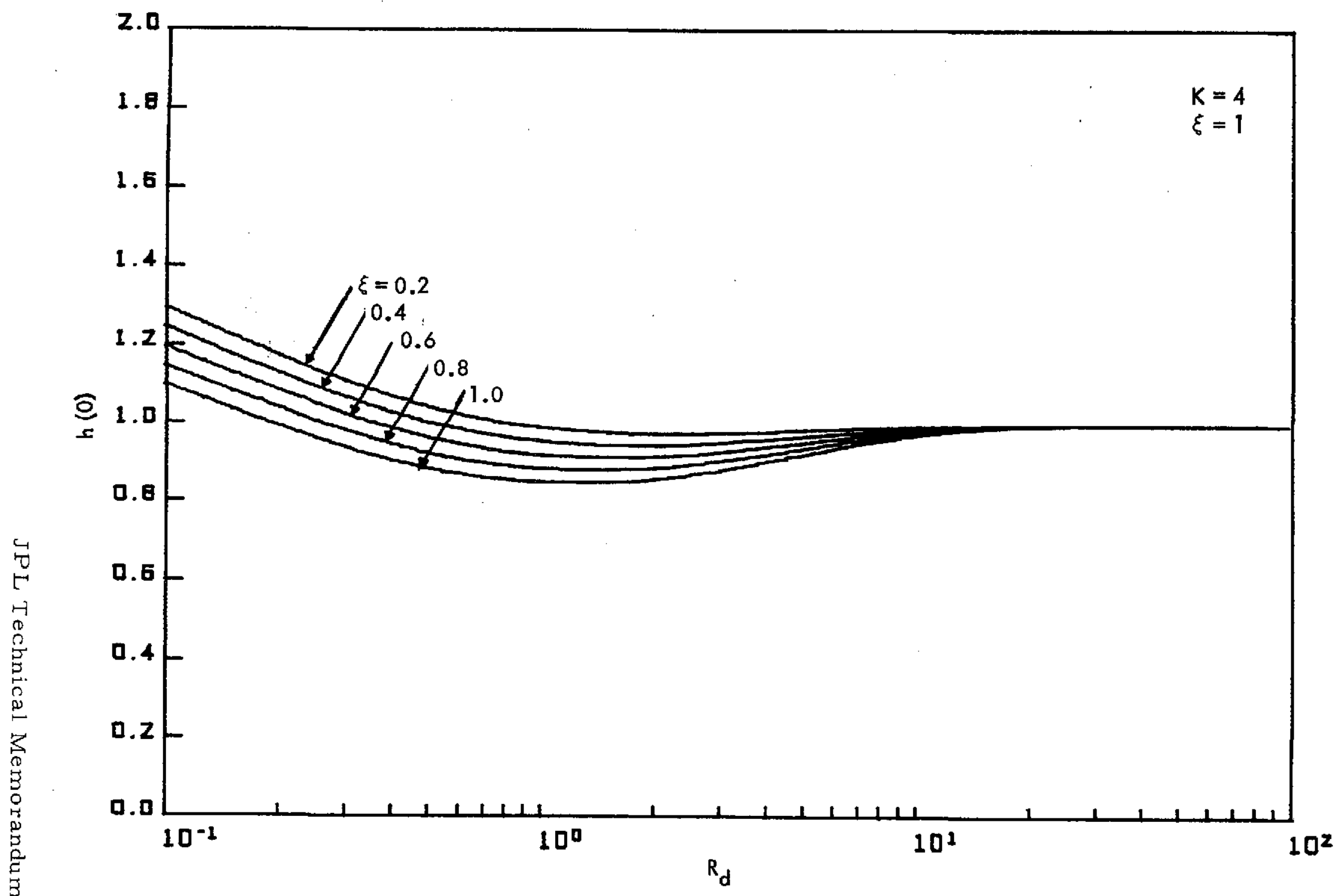

Fig. 6. Normalized Noise Spectral Density vs Signal-to-Noise Ratio; K=4 


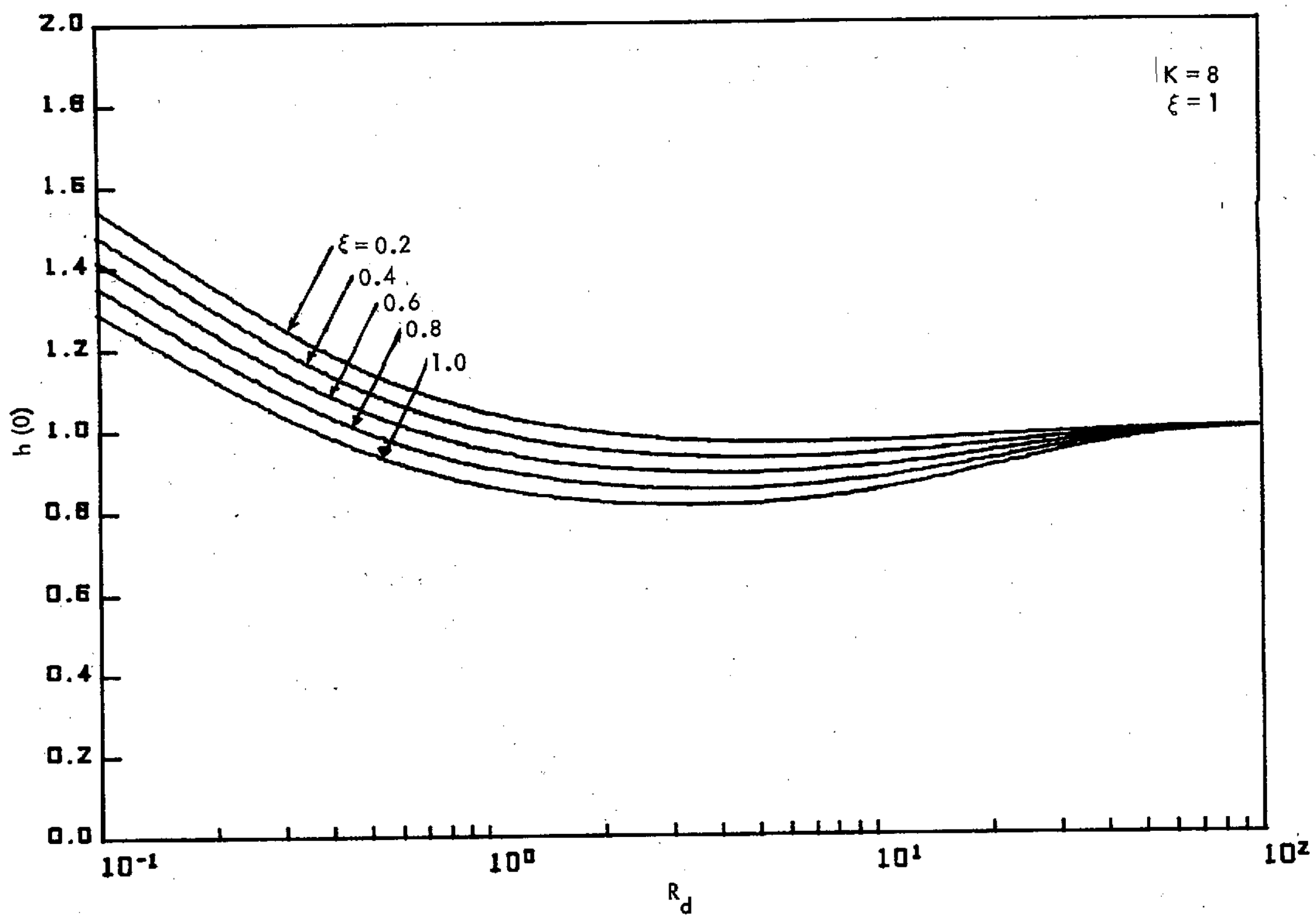




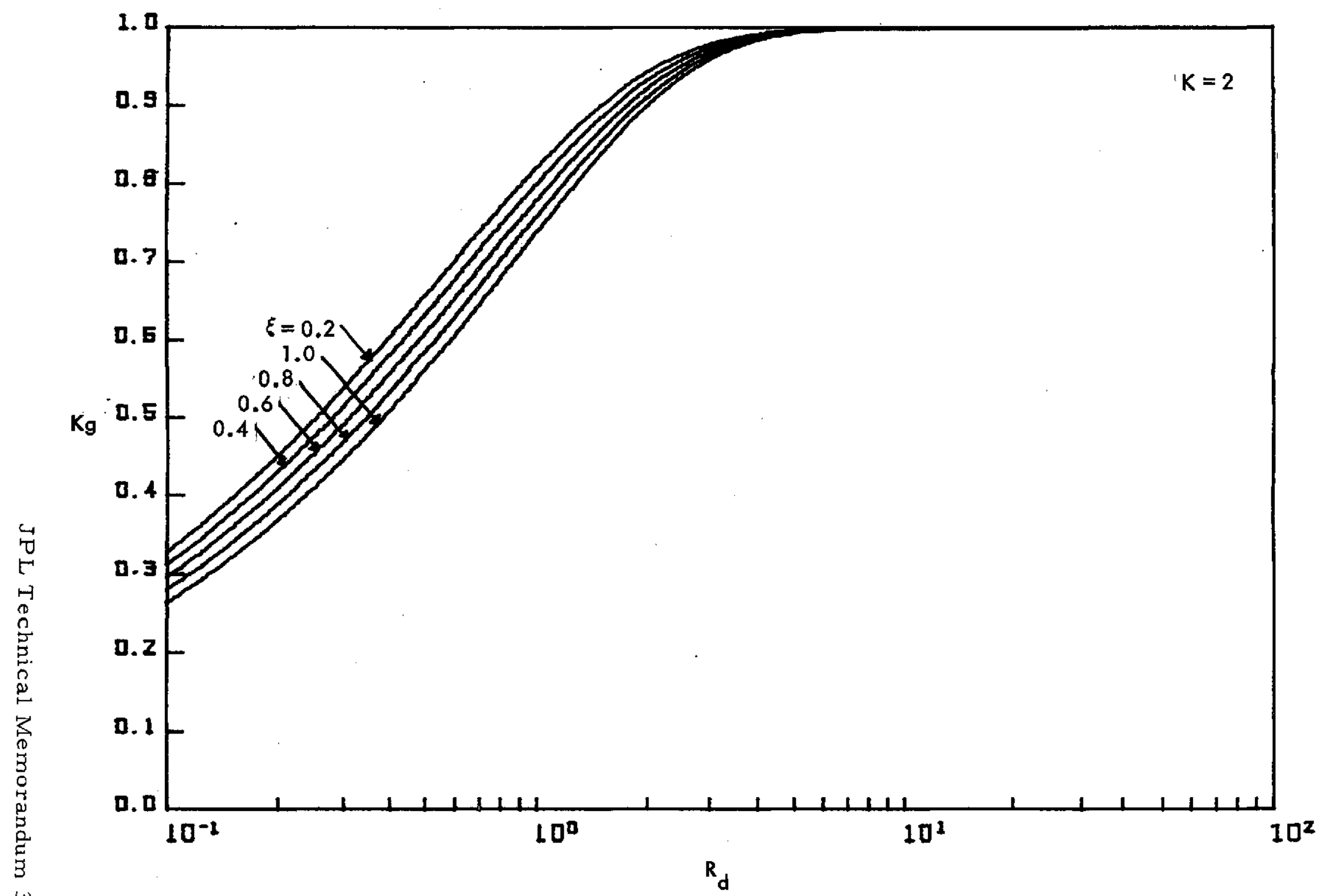

Fig. 8. Slope of S-Curve at Origin vs Signal-to-Noise Ratio; $K=2$ 


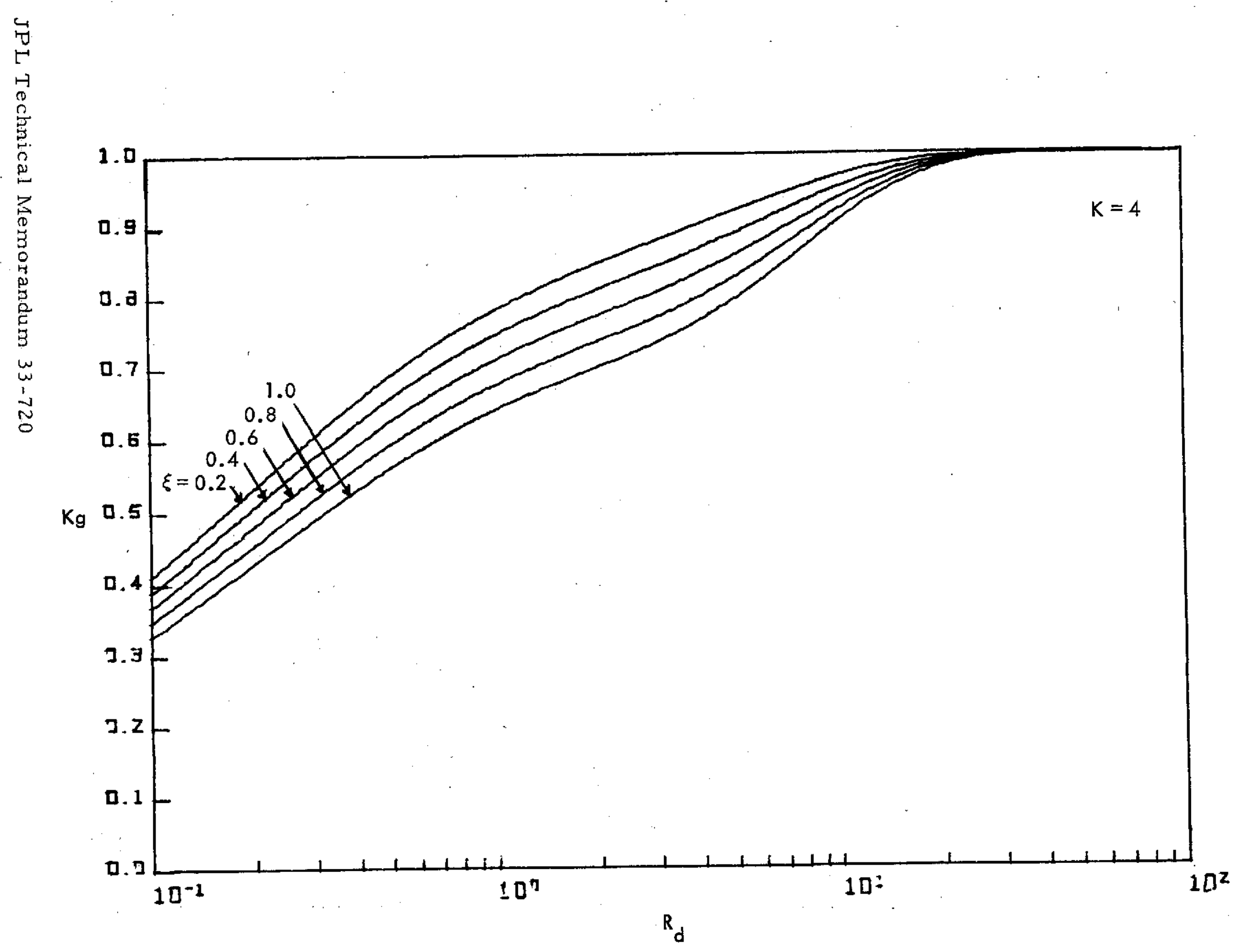

Fig. 9. Slope of S-Curve at Origin vs Signal-to-Noise Ratio; $K=4$ 


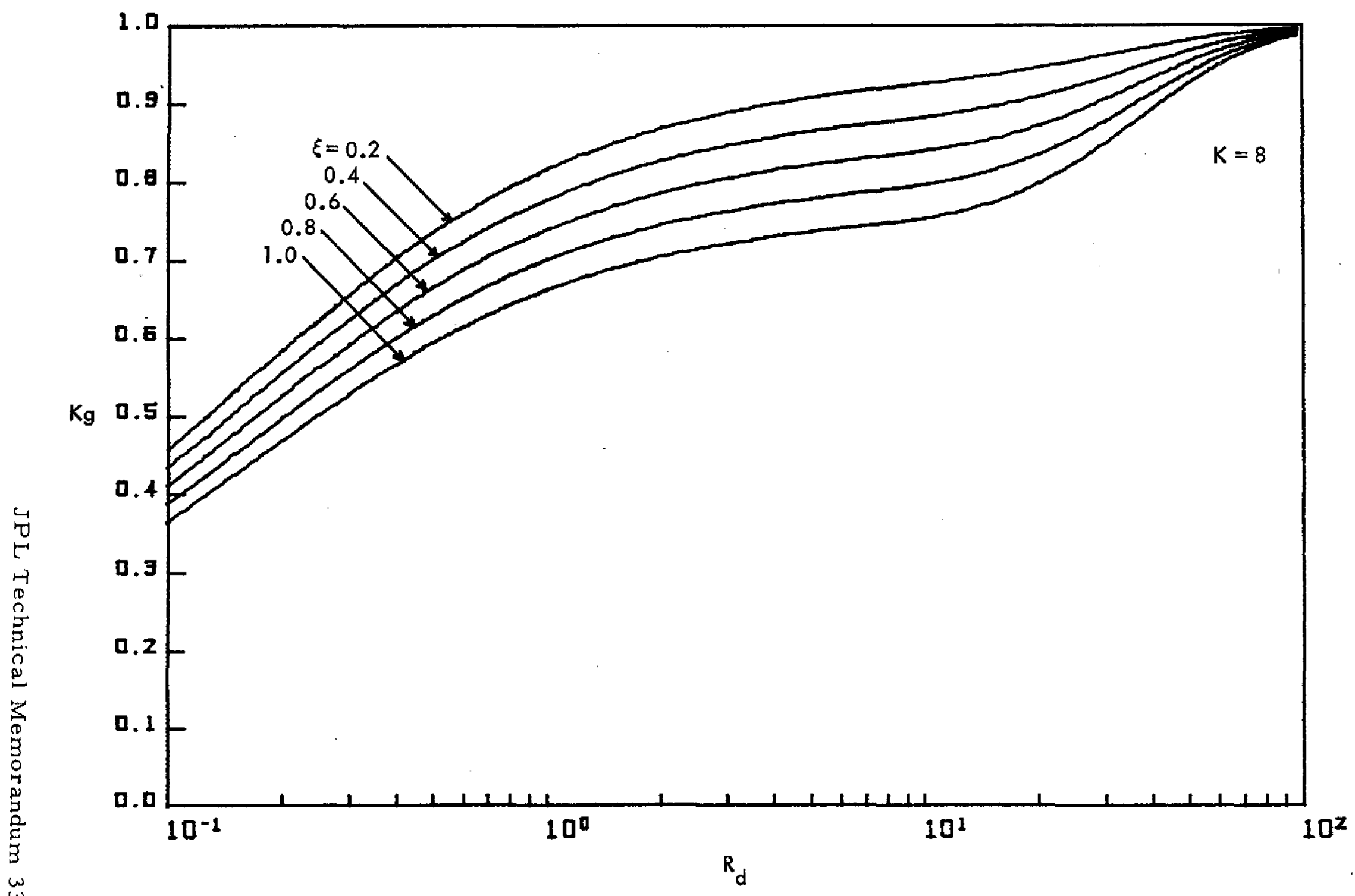

Fig. 10. Slope of S-Curve at Origin vs Signal-to-Noise Ratio; K=8 


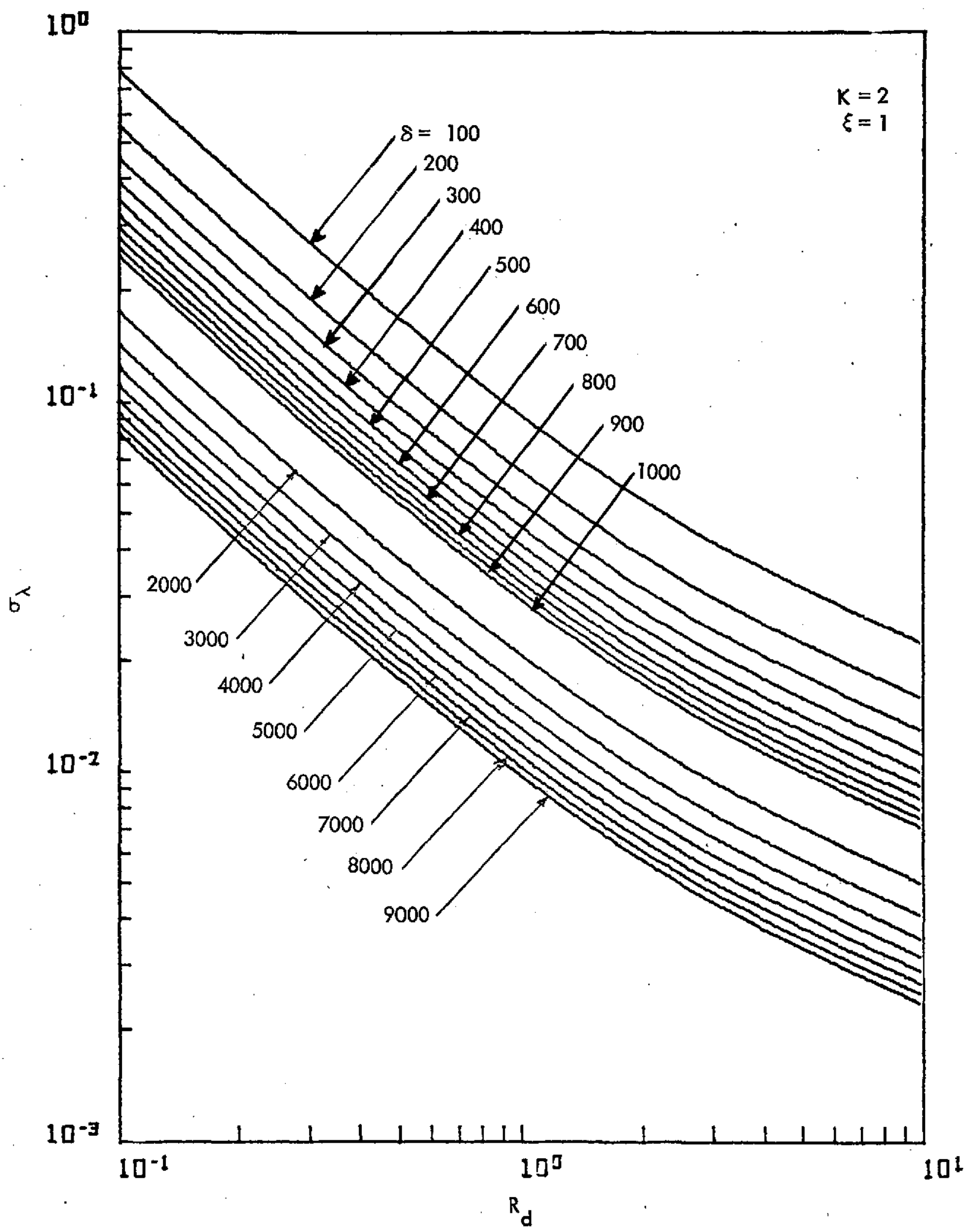

Fig. 11. RMS Symbol Sync Jitter vs Signal-to-Noise Ratio; K=2 


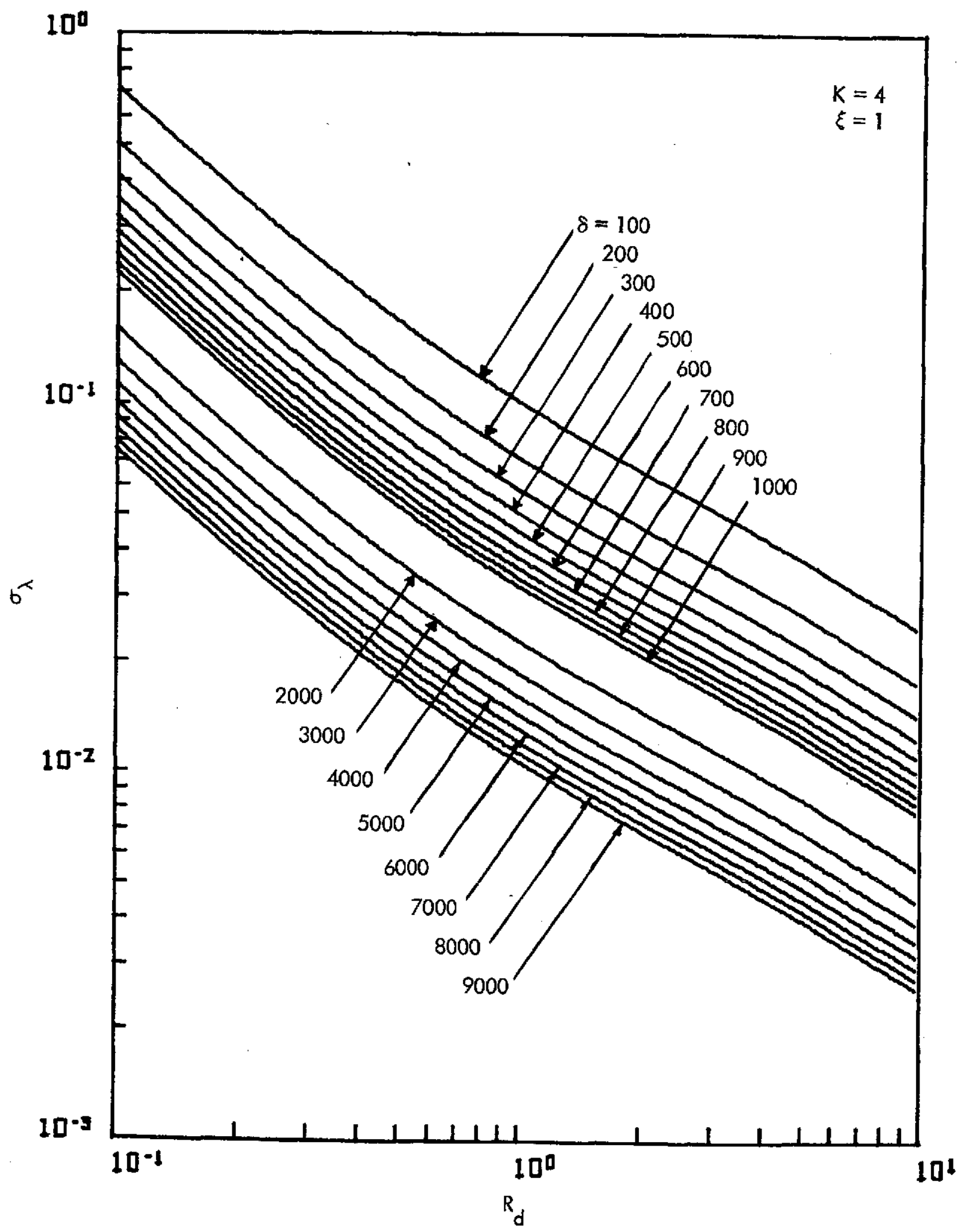

Fig. 12. RMS Symbol Sync Jitter vs Signal-to-Noise Ratio; K=4 


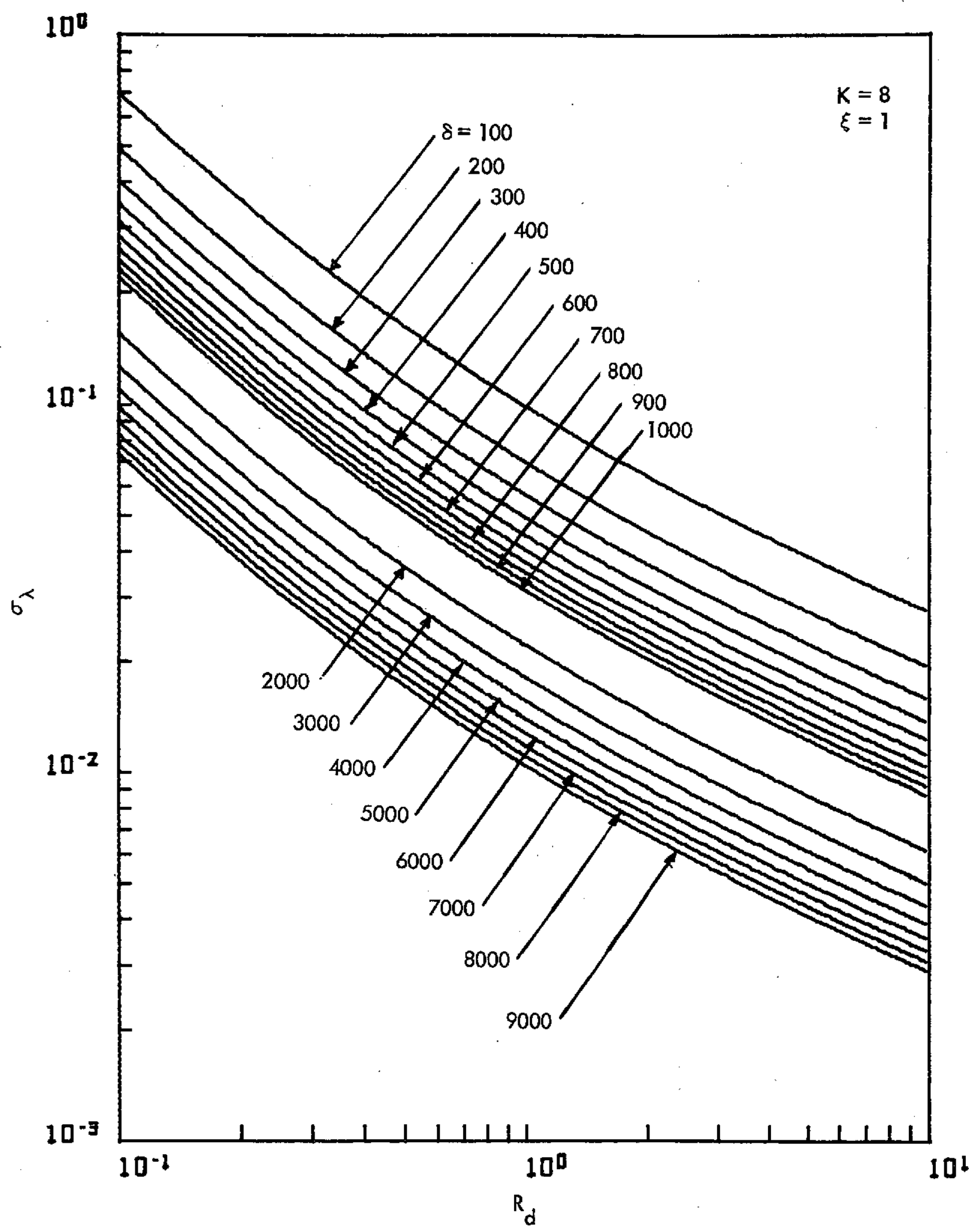

Fig. 13. RMS Symbol Sync Jitter vs Signal-to-Noise Ratio; K=8 


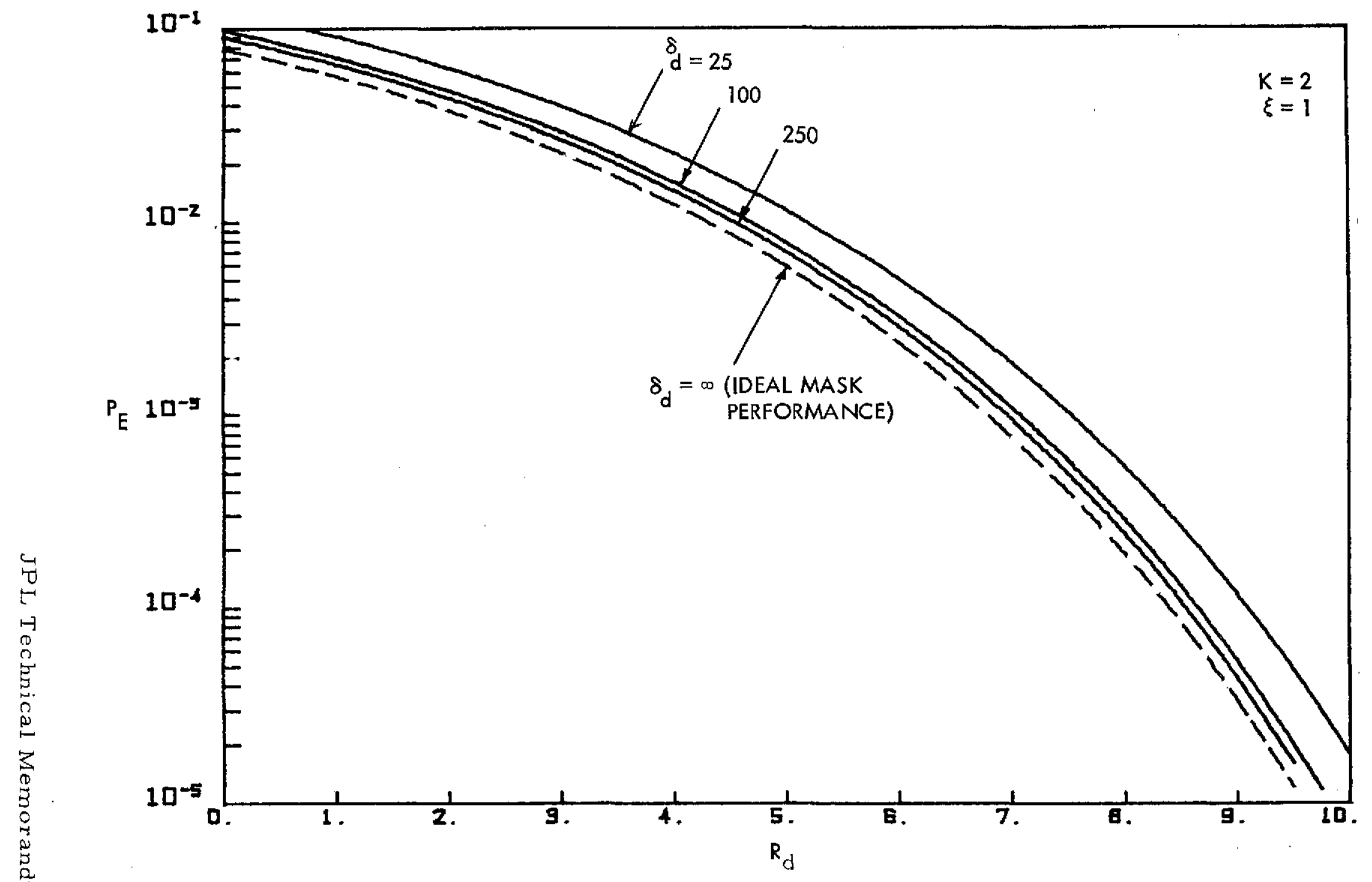

Fig. 14. Error Probability Performance of MASK System with Noisy Symbol Sync Reference; $K=2$ 


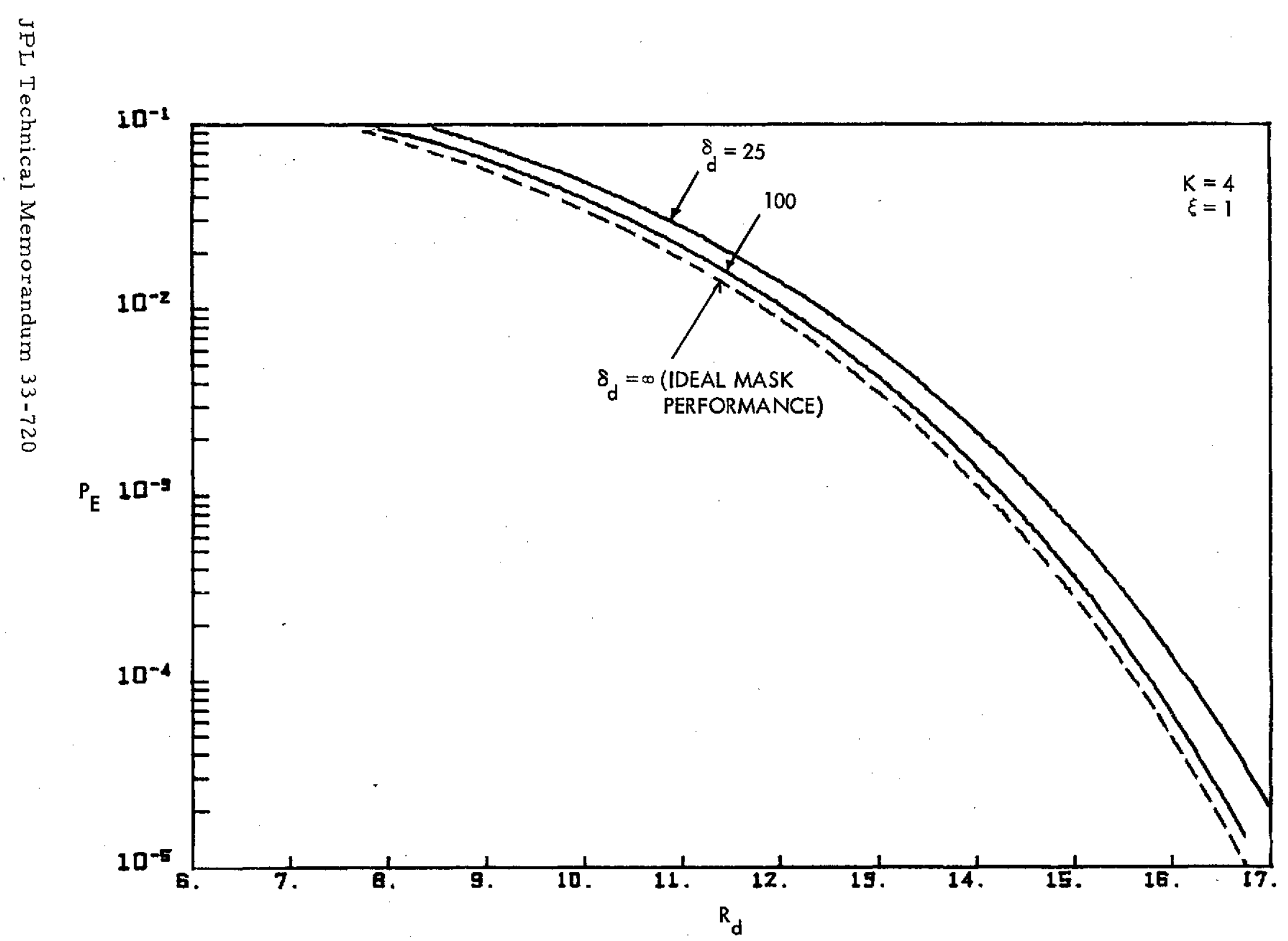

Fig. 15. Error Probability Performance of MASK System with Noisy 


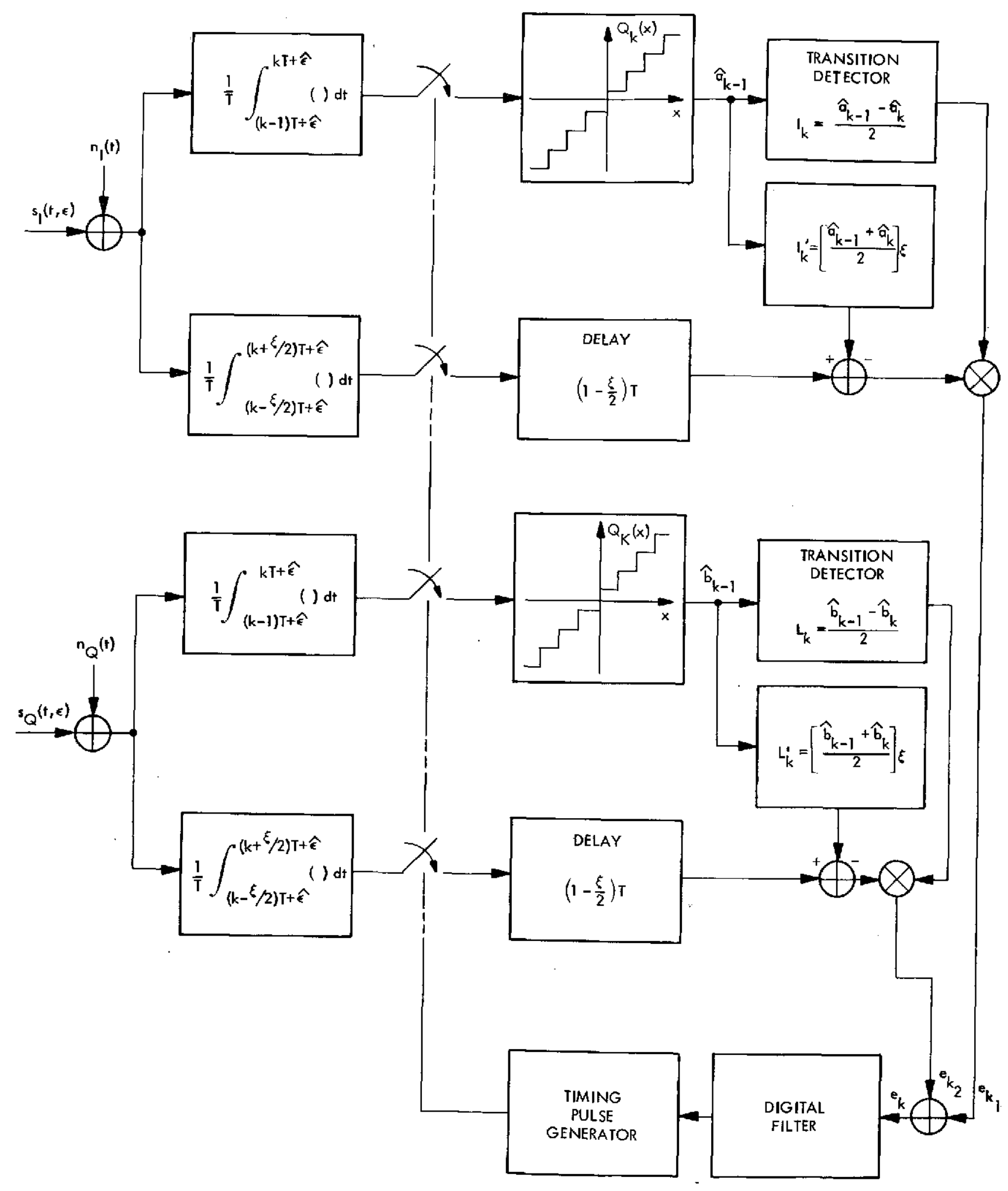

Fig. 16. Data-Transition Type of Symbol Synchronizer for QASK Signals 


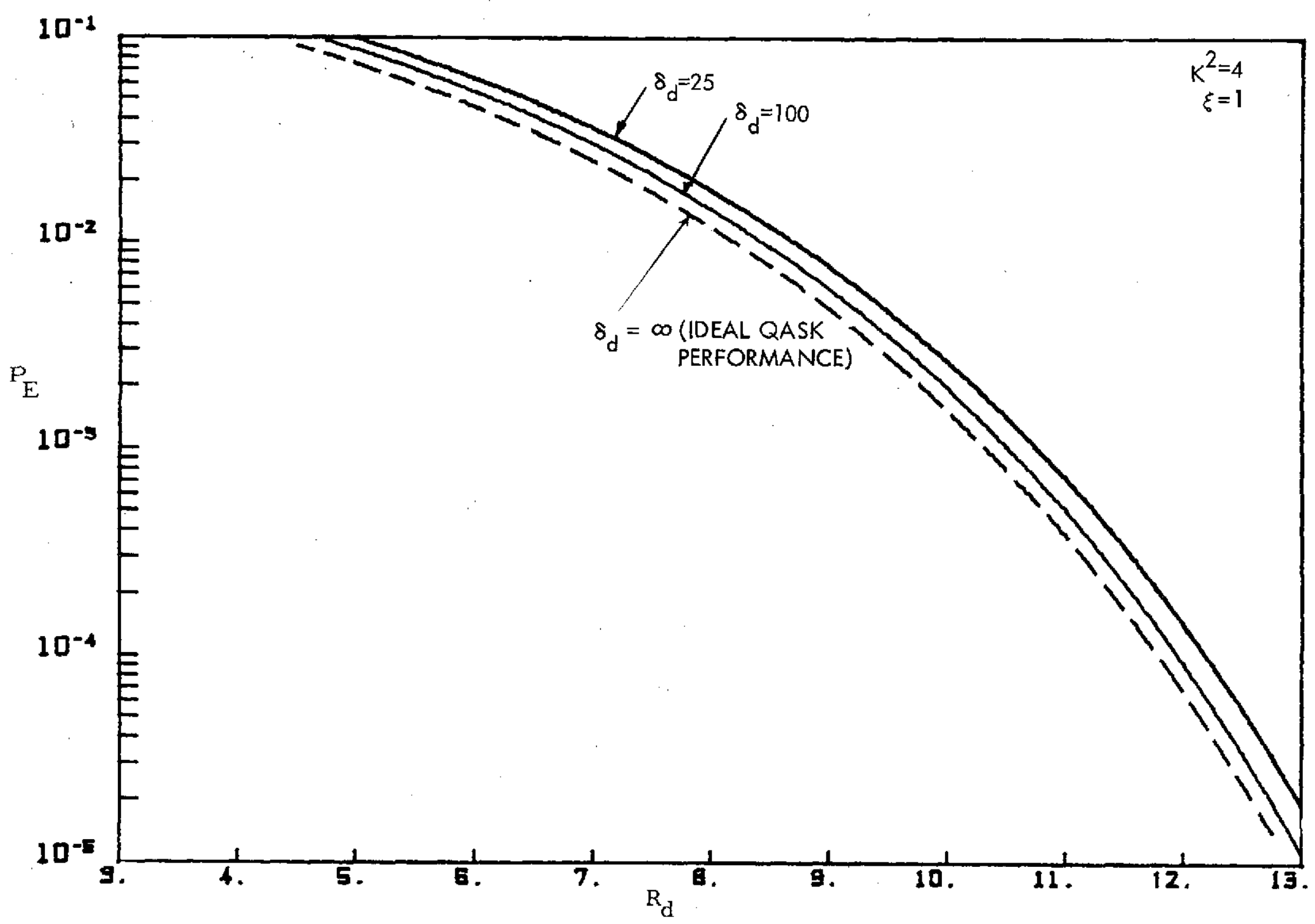

Fig. 17. Error Probability Performance of QASK System with Noisy Symbol Sync Reference; $\mathrm{K}^{2}=4$ (Quadriphase Signaling) 


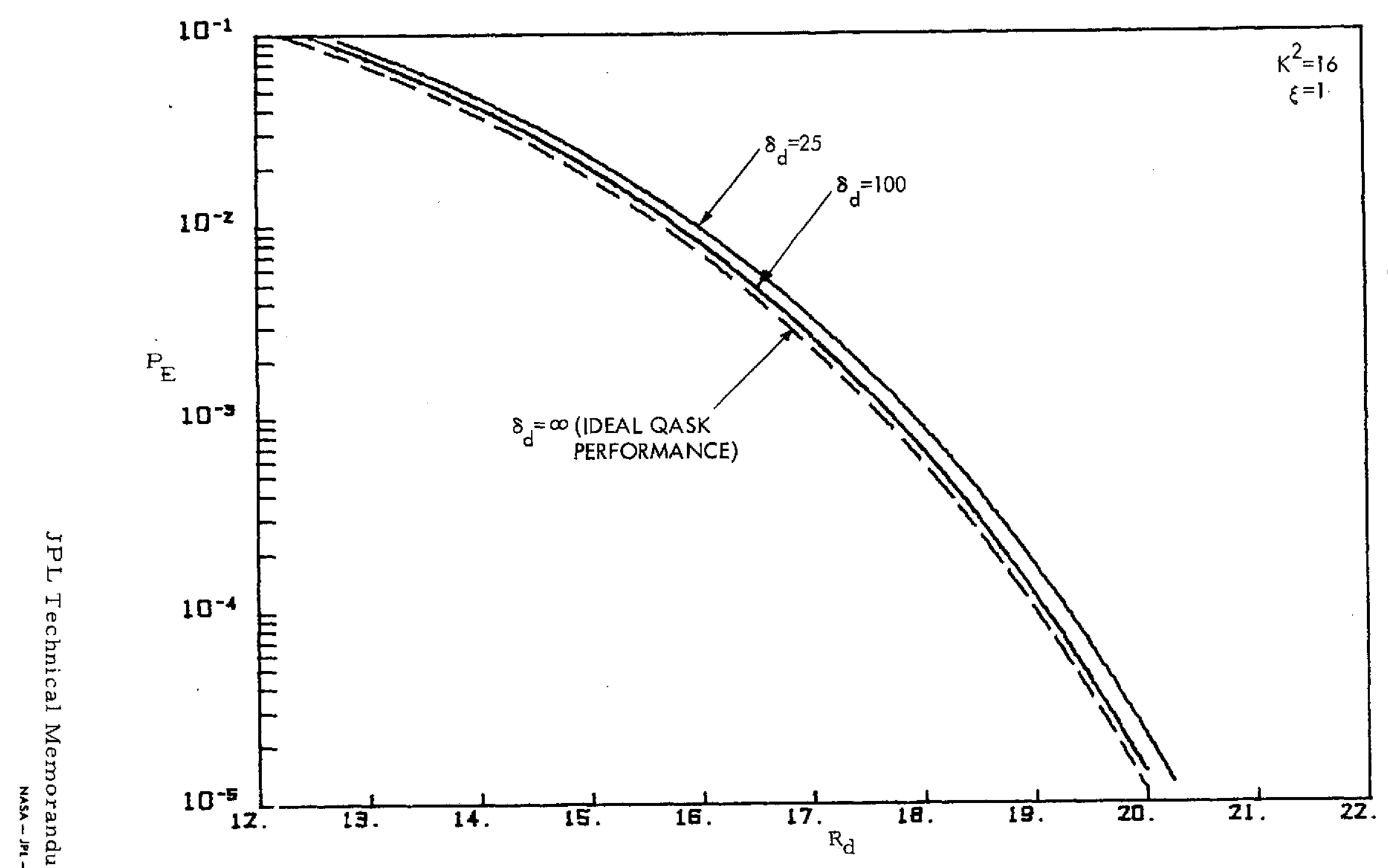

Fig. 18. Error Probability Performance of QASK System with Noisy Symbol Sync Reference; $\mathrm{K}^{2}=16$ 
Make items 1, 4, 5, 9, 12, and 13 agree with the corresponding information on the report cover. Use all capital letters for title (item 4). Leave items 2, 6, and 14 blank. Complete the remaining items as follows: .

3. Recipient's Catalog No. Reserved for use by report recipients.

7. Author(s). Include corresponding information from the report cover. In addition, list the affiliation of an author if it differs from that of the performing organization.

8. Performing Organization Report No. Insert if performing organization wishes' to assign this number.

10. Work Unit No. Use the agency-wide code (for example, 923-50-10-06-72), which uniquely identifies the work unit under which the work was authorized. Non-NASA performing organizations will leave this blank.

11. Insert the number of the contract or grant under which the report was prepared.

15. Supplementary Notes. Enter information not included elsewhere but useful, such as: Prepared in cooperation with... Translation of (or by)... Presented at conference of... To be published in...

16. Abstract. Include a brief (not to exceed 200 words) factual summary of the most significant information contained in the report." "If possible, the abstract of a classified report should be unclassified. If the report contains a significant bibliography or literature survey, mention it here.

17. Key Words, I Irisert terms or short phrases selected by the author that identify : : the principal subjects covered in the jeport, and that ore sufficiently specific and precise to be used for cataloging.

18. Distribution Statement. Enter'one of the authorized statements used to denote releasability to the public or a limitation on dissemination for reasons other "than security "of "defense information. Authorized statements "are "Unclassified-Unlimited," "U.S. Government and Contractors only,." "U. S. Government Ágencies only, " and "NASA and NASA Contractors only.".

19. Security Classification (of report). NOTE: Reports carrying a security. classification will require additional markings giving security and downgrading information as specified by the Security Requirements Checklist and the DoD Industrial Security Manual (DoD 5220.22-M).

20. Security Classification (of this page). NOTE: Because this page may be used in preparing announcements, bibliographies, and data banks, it should be unclassified if possible. If a classification is required, indicate separately the classification of the title and the abstract by following these items with either "(U)" for unclassified, or "(C)" or "(S)" as applicable for classified items. : : i

21. No. of Pages. Insert the number of pages.

22. Price. Insert the price set by the Clearinghouse for Federal Scientific and Technical Information or the Government Printing Office, if known. 
TECHNICAL REPORT STANDARD TITLE PAGE

\begin{tabular}{|c|c|c|}
\hline 1. Report No. 33-720 & 2. Government Accession No. & 3. Recipient's Catalog No. \\
\hline \multirow{2}{*}{\multicolumn{2}{|c|}{$\begin{array}{l}\text { 4. Title and Subtitle } \\
\text { DATA-DERIVED SYMBOL SYNCHRONIZATION OF } \\
\text { MASK AND QASK SIGNALS }\end{array}$}} & 5. Report Date \\
\hline & & 6. Performing Organization Code \\
\hline \multicolumn{2}{|l|}{ 7. Author(s) Marvin K. Simon } & 8. Performing Organization Report No. \\
\hline \multirow{3}{*}{\multicolumn{2}{|c|}{$\begin{array}{l}\text { 9. Performing Organization Name and Address } \\
\text { JET PROPULSION LABORATORY } \\
\text { California Institute of Technology } \\
\text { 4800 Oak Grove Drive } \\
\text { Pasadena, California } 91103\end{array}$}} & 10. Work Unit No. \\
\hline & & $\begin{array}{c}\text { 11. Contract or Grant No. } \\
\text { NAS } 7-100\end{array}$ \\
\hline & & \multirow{2}{*}{$\begin{array}{l}\text { 13. Type of Report and Period Covered } \\
\text { Technical Memorandum }\end{array}$} \\
\hline \multirow{2}{*}{\multicolumn{2}{|c|}{$\begin{array}{l}\text { 12. Sponsoring Agency Name and Address } \\
\text { NATIONAL AERONAUTICS AND SPACE ADMINISTRATION } \\
\text { Washington, D.C. } 20546\end{array}$}} & \\
\hline & & 14. Sponsoring Agency Code \\
\hline \multicolumn{3}{|l|}{ 15. Supplementary Notes } \\
\hline \multicolumn{3}{|c|}{$\begin{array}{l}\text { Much has been said in the literature with regard to the problem of estab- } \\
\text { Ifshing symbol symchronization in binary baseband digital communication } \\
\text { systems. By comparison, the literature is virtually devoid of information } \\
\text { relating to the extraction of symbol sync from multilevel baseband data. } \\
\text { With the recent interest in multilevel amplitude-shift-keying (MASK) and } \\
\text { quadrature amplitude-shift-keying (QASK) as signaling techniques for multi- } \\
\text { level digital communications systems, the problem of providing symbol } \\
\text { synchronization in the receivers of such systems becomes paramount. } \\
\text { This paper presents a technique for extracting symbol sync from an MASK } \\
\text { or QASK signal. The scheme is essentially a generalization of the data } \\
\text { transition tracking loop (DTTL) which has heretofore been used in PSK } \\
\text { systems. The performance of the loop is analyzed in terms of its mean- } \\
\text { squared symbol sync jitter and its effects on the data detection process } \\
\text { in MASK and QASK systems. }\end{array}$} \\
\hline $\begin{array}{l}\text { 17. Key Words (Selected by Author(s) } \\
\text { Tracking } \\
\text { Communication :Theory }\end{array}$ & 18. Distributi & \\
\hline $\begin{array}{l}\text { 19. Security Classif. (of this report) } \\
\text { Unclassified }\end{array}$ & $\begin{array}{l}\text { 20. Security Classif. (of this } \\
\text { Unclassified }\end{array}$ & \\
\hline
\end{tabular}

